UNITED STATES

DEPARTMENT OF THE INTERIOR

GEOLOGICAL SURVEY

DATA FOR WELLS AT THE LOW-LEVEL

RADIOACTIVE-WASTE BURIAL SITE IN THE

PALOS FOREST PRESERVE, ILLINOIS

By Julio C. Olimpio

U.S . GEOLOGICAL SURVEY

Open-File Report 82-692 


\section{UNITED STATES DEPARTMENT OF THE INTERIOR \\ JAMES G. WATT, Secretary}

\section{GEOLOGICAL SURVEY}

Dallas L. Peck, Director

For additional information write to:

District Chief, WRD

U.S. Geological Survey

4 th Floor

102 East Main street

Urbana, IL 61801
Copies of this report can be purchased from:

Open-File Services Section Western Distribution Branch U.S. Geological Survey Box 25425, Federal Center Lakewood, Colorado 80225 (Telephone: [303] 234-5888) 
Abstract . . . . . . . . . . . . . . . . . . . . 1

Introduction . . . . . . . . . . . . . . . . . . . . . . 1

Acknowledgments. . . . . . . . . . . . . . . . . . . 2

Drilling program . . . . . . . . . . . . . . . . . . . . 2

References . . . . . . . . . . . . . . . . . . 3

ILLUSTRATIONS

Figure 1. Map showing location of Plot $M$ in the Palos Forest

Preserve, Cook County, Illinois..........

2. Map of the Plot $M$ burial site showing test well

locations, topography, and intermittent streams...

TABLES

Table 1. Geologic time-stratigraphic classification, rockstratigraphic classification, and general description of the drift at the Plot M site.....

2. Test-well construction data and lithologic description of cores at Plot M. . . . . . . . . . . . .

3. Site and well-construction data for bedrock test wells in the forest preserve near Plot M. . . . . . . . 


\title{
DATA FOR WELLS AT THE LOW-LEVEL RADIOACTIVE-WASTE BURIAL SITE IN THE PALOS FOREST PRESERVE, ILLINOIS
}

\author{
by Julio C. Olimpio
}

\begin{abstract}
The U.S. Geological Survey is studying the geologic, hydrologic, and geochemical properties of the glacial drift and underlying bedrock at a low-level radioactive-waste burial site in the Palos Forest Preserve, 22 kilometers southwest of Chicago. Data collected from the 33 test wells drilled into the drift plus data from 4 wells drilled into the underlying dolomite bedrock are presented. Data include maps showing the Zocation of the test wells, a general description of the drift, well-construction information, and lithologic descriptions of cores from the wells finished in the drift.
\end{abstract}

\section{INTRODUCTION}

In early 1943, the U.S. Army Corps of Engineers leased land from the Cook County Forest Preserve District to build a radioisotope research facility. The facility formed part of the Metallurgical Laboratory operated by the University of Chicago for the Manhattan Engineer District (the Manhattan Project). The facility, known as Site A (fig. 1), housed the world's first nuclear reactor. Plot $M$ was the burial site for the low-level radioactive waste resulting from operations at the Metallurgical Laboratory.

The Argonne National Laboratory (ANL) has conducted a radiological monitoring program at Plot $M$ since 1948. In 1954, at the request of $A N L$, the U.S. Geological Survey (USGS) studied the geologic, hydrologic, and chemical characteristics of the glacial drift at Plot $M$ (W. J. Drescher, written commun., 1954) and advised ANL on its environmental monitoring program. In 1973, tritium was detected in water from a dolomite bedrock well at the Red Gate Woods picnic area. Consequently, the ANL monitoring program was expanded to include the Red Gate Woods well and other nearby forest preserve wells.

This study of ground-water flow and tritium migration at Plot $M$ began in 1978. The two main objectives of the study are to determine the geologic, hydrologic, and geochemical properties of the drift that control the migration of tritium from the burial site to the underlying bedrock and to determine the 
present extent of tritium in the drift. A third objective is to determine the rate and direction of tritium movement in water in the bedrock. The results of the study are needed by the U.S. Department of Energy to evaluate the need for remedial action at the Plot $M$ site. Several test wells were drilled to help accomplish these objectives.

The purpose of this report is to present the physical description of each well and a description of the lithologic materials encountered.

\section{ACKNOWLEDGMENTS}

This investigation relied heavily on the Plot $M$ environmental surveillance program by the Argonne National Laboratory. Especially helpful have been J. Sedlet, N. W. Golchert, and H. C. Svoboda of the Health Safety Section of ANL who have provided administrative and technical assistance throughout the project.

\section{DRILLING PROGRAM}

The Plot $M$ site is situated on a moraine and is directly underlain by 25-30 meters of clayey silt. The clayey silt, which is the Wadsworth Till Member (of Willman and Frye, 1970) of the Wedron Formation, contains thin lenses and layers of sand and gravel and overlies sandy silt and gravel of the Malden Till Member (of Willman and Frye, 1970) of the Wedron Formation. The total thickness of the drift beneath the site ranges from 25 to 45 meters.

In 1976, under USGS supervision, ANL began construction of a network of test wells in the drift (fig. 2). Drilling at each site was accompanied by continuous split-spoon sampling to identify variations in lithologic properties. Cores and samples were logged in the field and sealed for laboratory analysis. Field observations were made of the lithology, texture, color, structure, and sedimentologic features of each sample. Vertical variations in lithology, grain-size distribution, and color were observed within both the Wadsworth and Malden tills. These vertical variations in the character of the drift were consistent at all test wells and were used to categorize the drift into seven lithostratigraphic units. The units are referred to, from the surface downward, as Units 1 through 7 (table 1 ). Units 1 through 5 represent the Wadsworth Till Member and Units 6 and 7 represent the Malden Till Member.

Table 2 includes information on the length of core recovered and the geologic log of each well finished in the drift. Table 3 includes the wellcompletion information for the four bedrock test wells drilled near Plot $M$ (fig. 1) in 1976. A complete description of the techniques used to drill the pre-1979 wells, together with the results of the radionuclide analyses of all the core samples collected, is given by Golchert and sedlet (1978). 


\section{REFERENCES}

Golchert, N. W., and Sedlet, Jacob, 1978, Radiological survey of Site A and Plot M: U.S. Department of Energy Report DOE/EV-0005/7, 89 p.

Willman, H. B., and Frye, J. C., 1970, Pleistocene stratigraphy of Illinois: Illinois State Geological Survey Bulletin 94, 204 p. 
FIGURES 1 and 2; TABLES 1 to 3 


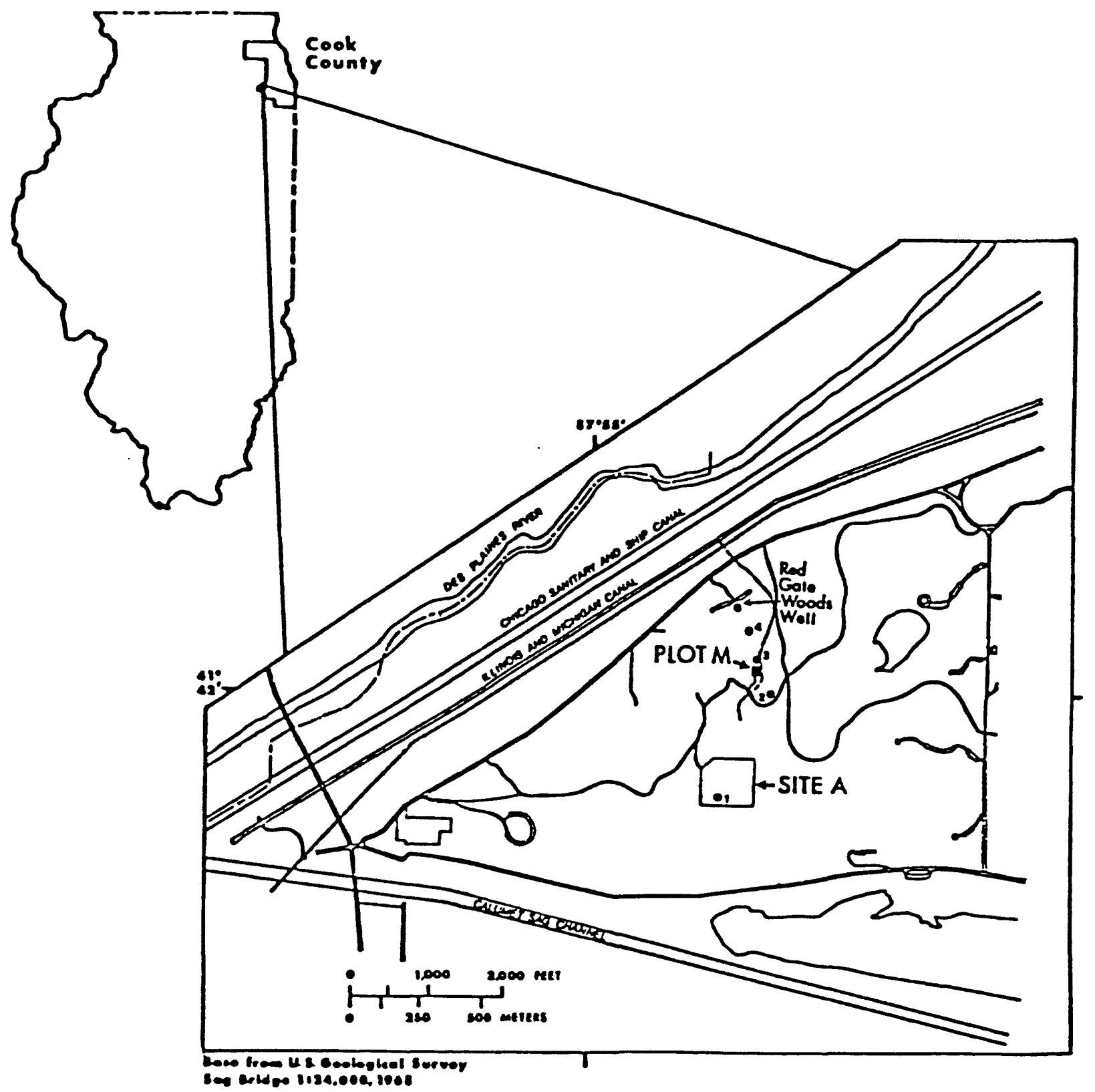

Figure 1.--Location of Plot $M$ in the Palos Forest Preserve, Cook County, Illinois. Wells 1 through 4 and the Red Gate Woods well are finished in the underlying dolomite bedrock. 


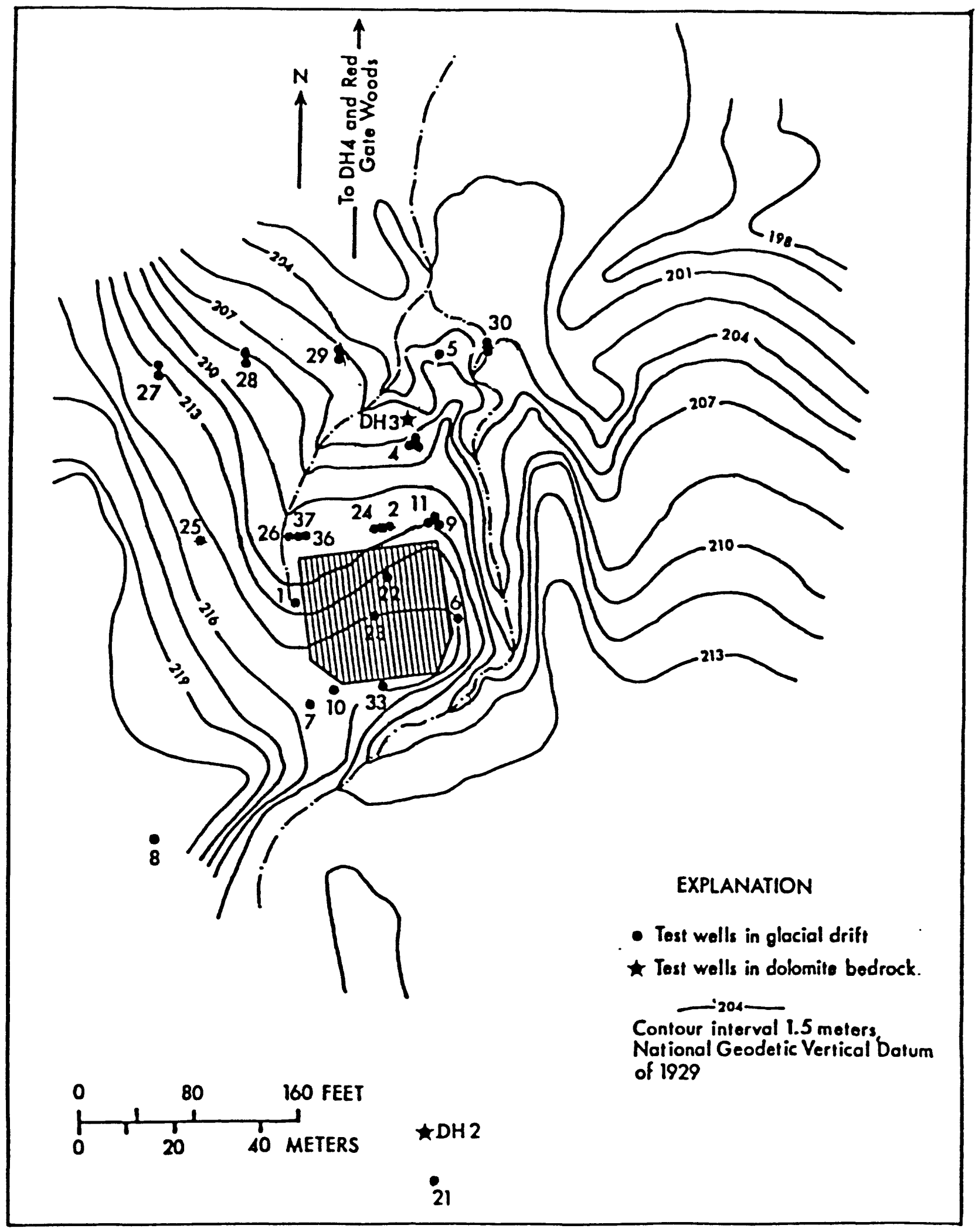

Figure 2.--The Plot M burial site showing test-well locations, topography, and intermittent streams. 


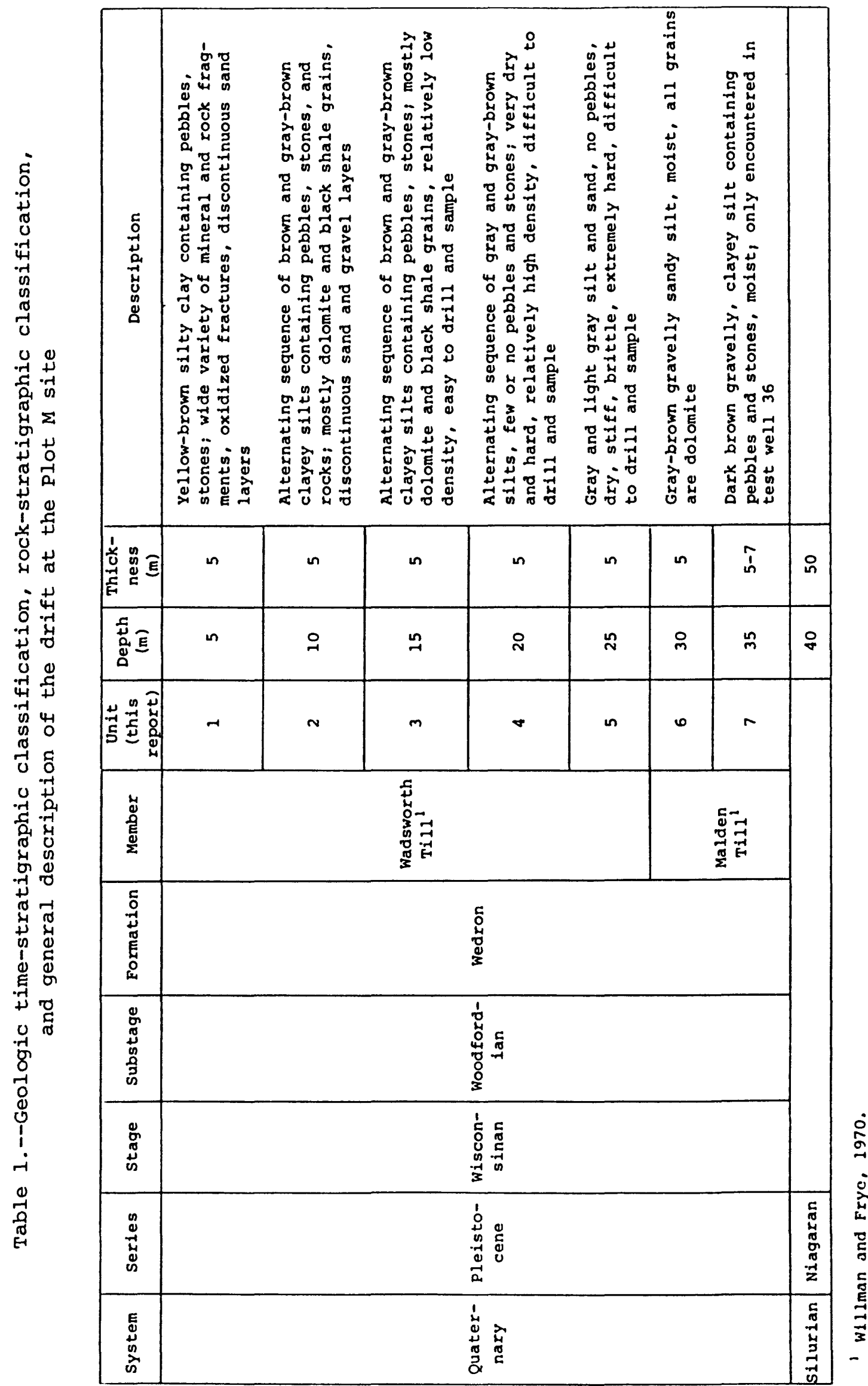


Table 2.--Test-well construction data and lithologic description of cores at Plot $M$

Test well 1

Completion date: March 31, 1976

Location: 26 meters west of the center of Plot M

Site and well construction data:

Altitude of land surface . . . . . . . . . . $213.81 \mathrm{~m}$

Altitude of measuring point (top of casing). . . . . $213.81 \mathrm{~m}$

Depth to bottom of piezometer from land surface. . . $12.19 \mathrm{~m}$

Type of piezometer: $1.52 \mathrm{~m}$ perforated PVC pipe, $6.35 \mathrm{~cm}$ diameter

\begin{tabular}{|c|c|c|c|c|c|}
\hline $\begin{array}{l}\text { Unit } \\
\text { No. }\end{array}$ & $\begin{array}{l}\text { Sample } \\
\text { No. }\end{array}$ & $\begin{array}{c}\text { Depth } \\
\text { interval } \\
\text { (meters) }\end{array}$ & $\begin{array}{l}\text { Blow } \\
\text { counts }\end{array}$ & $\begin{array}{l}\text { Recovery } \\
\quad(\mathrm{cm})\end{array}$ & Description \\
\hline 1 & 7657 & $0-0.46$ & & 27.9 & $\begin{array}{l}\text { black topsoil over brown silty clay } \\
\text { w/pebbles, plant fragments }\end{array}$ \\
\hline 1 & 7658 & $0.46-0.91$ & & 33.0 & brown silty clay $w /$ pebbles and stones \\
\hline 1 & 7659 & $0.91-1.37$ & & 24.8 & grayish brown clay $\mathrm{w} / \mathrm{small}$ stones \\
\hline 1 & & $1.37-1.83$ & & & \\
\hline 1 & $76 \mathrm{~S} 10$ & $1.83-2.29$ & & 27.9 & $\begin{array}{l}\text { grayish brown silty clay grading to } \\
\text { gray silty clay }\end{array}$ \\
\hline 1 & & $2.29-2.74$ & & & \\
\hline 1 & $76 \mathrm{~S} 11$ & $2.74-3.20$ & & 11.4 & $\begin{array}{l}\text { brown silty clay } w / \text { large stones; } \\
\text { numerous stones at bottom }\end{array}$ \\
\hline 1 & & $3.20-3.66$ & & & \\
\hline 1 & $76 \mathrm{Sl2}$ & $3.66-4.12$ & & 21.6 & brown silty clay w/stones \\
\hline 1 & & $4.12-4.57$ & & & \\
\hline 1 & $76 S 13$ & $4.57-5.03$ & & 22.9 & $\begin{array}{l}\text { brown silty clay to } 4.72 \mathrm{~m} \text {; sand at } \\
4.72 \mathrm{~m} \mathrm{w} / \mathrm{stones}\end{array}$ \\
\hline 1 & & $5.03-5.49$ & & & \\
\hline 1 & & $5.49-5.94$ & & & \\
\hline
\end{tabular}


Table 2.--Test-well construction data and lithologic description of cores at Plot M--Continued

Test well 1--Continued

\begin{tabular}{|c|c|c|c|c|c|}
\hline $\begin{array}{l}\text { Unit } \\
\text { No. }\end{array}$ & $\begin{array}{l}\text { Sample } \\
\text { No. }\end{array}$ & $\begin{array}{c}\text { Depth } \\
\text { interval } \\
\text { (meters) }\end{array}$ & $\begin{array}{l}\text { Blow } \\
\text { counts }\end{array}$ & $\begin{array}{l}\text { Recovery } \\
\quad(\mathrm{cm})\end{array}$ & Description \\
\hline 1 & $76 S 14$ & $5.94-6.40$ & & 40.6 & $\begin{array}{l}\text { brown clayey silt } \mathrm{w} / \text { stones, large } \\
\text { variety of rock and mineral grains }\end{array}$ \\
\hline 1 & & $6.40-6.86$ & & & \\
\hline 1 & & $6.86-7.32$ & & & \\
\hline 2 & & $7.32-7.77$ & & & \\
\hline 2 & $76 S 15$ & $7.77-8.23$ & & 38.1 & brownish gray clayey silt $\mathrm{w} /$ stones \\
\hline 2 & & $8.23-8.69$ & & & \\
\hline 2 & & $8.69-9.14$ & & & \\
\hline 2 & $76 S 16$ & $9.14-9.60$ & & 30.5 & $\begin{array}{l}\text { gray clayey silt } w / \text { limestone pebbles, } \\
\text { stones, firm, moist }\end{array}$ \\
\hline 2 & & $9.60-10.06$ & & & \\
\hline 2 & & $10.06-10.52$ & & & \\
\hline 2 & $76 S 17$ & $10.52-10.97$ & & 27.9 & gray clayey silt $w /$ limestone pebbles \\
\hline 2 & & $10.97-11.43$ & & & \\
\hline 2 & & $11.43-11.89$ & & & \\
\hline 2 & & $11.89-12.34$ & & & \\
\hline 2 & $76 S 18$ & $12.34-12.64$ & $18-34-27$ & 25.4 & $\begin{array}{l}\text { gray clay silt w/pebbles, firm, moist } \\
\text { to wet; sand partings }\end{array}$ \\
\hline & & EOB $12.64 \mathrm{~m}$ & & & \\
\hline
\end{tabular}


Table 2.--Test-well construction data and lithologic description of cores at Plot $\mathrm{M}--$ Continued

\section{Test well 2}

Completion date: April 6, 1976

Location: 26 meters north of the center of Plot $M$

Site and well construction data:

Altitude of land surface . . . . . . . . . . $211.07 \mathrm{~m}$

Altitude of measuring point (top of casing)...... $211.13 \mathrm{~m}$

Depth to bottom of piezometer from land surface. . . . $12.13 \mathrm{~m}$

Type of piezometer: $1.52 \mathrm{~m}$ perforated PVC pipe, $6.35 \mathrm{~cm}$ diameter

\begin{tabular}{|c|c|c|c|c|c|}
\hline $\begin{array}{l}\text { Unit } \\
\text { No. }\end{array}$ & $\begin{array}{l}\text { Sample } \\
\text { No. }\end{array}$ & $\begin{array}{c}\text { Depth } \\
\text { interval } \\
\text { (meters) }\end{array}$ & $\begin{array}{l}\text { Blow } \\
\text { counts }\end{array}$ & $\begin{array}{l}\text { Recovery } \\
\quad(\mathrm{cm})\end{array}$ & Description \\
\hline 1 & $76 \$ 41$ & $0-0.46$ & & 35.6 & brown silty clay topsoil w/stones \\
\hline 1 & $76 S 42$ & $0.46-0.91$ & & 22.9 & brown silty clay $w /$ pebbles, stones \\
\hline 1 & 76543 & $0.91-1.37$ & & 22.9 & brown silty clay w/pebbles, stones \\
\hline 1 & & $1.37-1.83$ & & & \\
\hline 1 & $76 S 44$ & $1.83-2.29$ & & 25.4 & $\begin{array}{l}\text { brown silty clay w/pebbles, stones, } \\
\text { mottled }\end{array}$ \\
\hline 1 & & $2.29-2.74$ & & & \\
\hline 1 & & $2.74-3.20$ & & & \\
\hline 1 & $76 \$ 45$ & $3.20-3.66$ & & 35.6 & brown silty clay w/pebbles, stones \\
\hline 1 & & $3.66-4.12$ & & & \\
\hline 1 & & $4.12-4.57$ & & & \\
\hline 1 & $76 S 46$ & $4.57-5.03$ & & 35.6 & brown silty clay w/pebbles, stones \\
\hline 1 & & $5.03-5.49$ & & & \\
\hline 1 & & $5.49-5.94$ & & & \\
\hline 1 & $76 \$ 47$ & $5.94-6.40$ & & 55.9 & $\begin{array}{l}\text { brown gray silty clay to } 6.25 \mathrm{~m} \text {; con- } \\
\text { tact w/brown clayey silt }\end{array}$ \\
\hline
\end{tabular}


Table 2.--Test-well construction data and lithologic description of cores at Plot M--Continued

Test well 2--Continued

\begin{tabular}{|c|c|c|c|c|c|}
\hline $\begin{array}{l}\text { Unit } \\
\text { No. }\end{array}$ & $\begin{array}{c}\text { Sample } \\
\text { No. }\end{array}$ & $\begin{array}{c}\text { Depth } \\
\text { interval } \\
\text { (meters) }\end{array}$ & $\begin{array}{c}\text { Blow } \\
\text { counts }\end{array}$ & $\begin{array}{l}\text { Recovery } \\
(\mathrm{cm})\end{array}$ & Description \\
\hline 1 & & $6.40-6.86$ & & & \\
\hline 2 & & $6.86-7.32$ & & & \\
\hline 2 & $76 S 48$ & $7.32-7.77$ & & 27.9 & $\begin{array}{l}\text { brown clayey silt to } 7.42 \mathrm{~m} \text {; contact } \\
\text { w/gray clayey silt w/pebbles, mostly } \\
\text { dolomite }\end{array}$ \\
\hline 2 & & $7.77-8.23$ & & & \\
\hline 2 & & $8.23-8.69$ & & & \\
\hline 2 & & $8.69-9.14$ & & & \\
\hline 2 & $76 \$ 49$ & $9.14-9.60$ & & 43.2 & $\begin{array}{l}\text { gray clayey silt w/pebbles to } 9.54 \mathrm{~m} \text {; } \\
1.3 \mathrm{~cm} \text { sand layer at } 9.54 \mathrm{~m} \text {; contact } \\
\text { w/brownish gray clayey silt }\end{array}$ \\
\hline 2 & & $9.60-10.06$ & & & \\
\hline 2 & & $10.06-10.52$ & & & \\
\hline 2 & $76 S 50$ & $10.52-10.97$ & & 22.9 & $\begin{array}{l}\text { gray clayey silt w/pebbles, moist to } \\
\text { wet to } 10.82 \mathrm{~m} ; 15.2 \mathrm{~cm} \text { water-bearing } \\
\text { sand and gravel w/clayey silt at } \\
10.82 \mathrm{~m}\end{array}$ \\
\hline 2 & & $10.97-11.43$ & & & \\
\hline 2 & & $11.43-11.89$ & & & \\
\hline 2 & $76 S 51$ & $11.89-12.34$ & & 7.6 & gray clayey silt \\
\hline
\end{tabular}


Table 2.--Test-well construction data and lithologic description of cores at Plot M--Continued

\section{Test well 3}

\section{Completion date: April 7, 1976}

\section{Location: 7 meters north of the northeast corner of Plot $M$}

\section{Site and well construction data:}

Altitude of land surface ............. . $211.31 \mathrm{~m}$

Altitude of measuring point (top of casing). . . . . $211.31 \mathrm{~m}$

Depth to bottom of piezometer from land surface. . . $12.19 \mathrm{~m}$

Type of piezometer: $1.52 \mathrm{~m}$ perforated PVC pipe, $6.35 \mathrm{~cm}$ diameter

\begin{tabular}{|c|c|c|c|c|c|}
\hline $\begin{array}{l}\text { Unit } \\
\text { No. }\end{array}$ & $\begin{array}{c}\text { Sample } \\
\text { No. }\end{array}$ & $\begin{array}{c}\text { Depth } \\
\text { interval } \\
\text { (meters) }\end{array}$ & $\begin{array}{l}\text { Blow } \\
\text { counts }\end{array}$ & $\begin{array}{l}\text { Recovery } \\
\quad(\mathrm{cm})\end{array}$ & Description \\
\hline 1 & 76563 & $0-0.46$ & & 27.9 & $\begin{array}{l}\text { brown silty clay w/grass, roots, } \\
\text { plant fragments, stones }\end{array}$ \\
\hline 1 & $76 \$ 64$ & $0.46-0.91$ & & 33.0 & brown silty clay w/pebbles \\
\hline 1 & $76 \$ 65$ & $0.91-1.37$ & & 30.5 & brown silty clay w/pebbles \\
\hline 1 & & $1.37-1.83$ & & & \\
\hline 1 & $76 \$ 66$ & $1.83-2.29$ & & 22.9 & brown clayey silt w/pebbles \\
\hline 1 & & $2.29-2.74$ & & & \\
\hline 1 & $76 S 67$ & $2.74-3.20$ & & 16.5 & brown clayey silt $w /$ pebbles \\
\hline 1 & & $3.20-3.66$ & & & \\
\hline 1 & & $3.66-4.12$ & & & \\
\hline 1 & & $4.12-4.57$ & & & \\
\hline 1 & $76 \$ 68$ & $4.57-5.03$ & & 26.7 & brown clayey silt $w /$ pebbles \\
\hline 1 & & $5.03-5.49$ & & & \\
\hline 1 & & $5.49-5.94$ & & & \\
\hline 2 & $76 \$ 69$ & $5.94-6.40$ & & 27.9 & brown clayey silt $w /$ pebbles \\
\hline
\end{tabular}


Table 2.--Test-well construction data and lithologic description of cores at Plot $\mathrm{M}$--Continued

Test well 3--Continued

\begin{tabular}{|c|c|c|c|c|c|}
\hline $\begin{array}{l}\text { Unit } \\
\text { No. }\end{array}$ & $\begin{array}{l}\text { Sample } \\
\text { No. }\end{array}$ & $\begin{array}{c}\text { Depth } \\
\text { interval } \\
\text { (meters) }\end{array}$ & $\begin{array}{l}\text { Blow } \\
\text { counts }\end{array}$ & $\begin{array}{l}\text { Recovery } \\
\quad(\mathrm{cm})\end{array}$ & Description \\
\hline 2 & & $6.40-6.86$ & & & \\
\hline 2 & & $6.86-7.32$ & & & \\
\hline 2 & $76 S 70$ & $7.32-7.77$ & & 11.4 & $\begin{array}{l}\text { gray clayey silt w/pebbles, stones, } \\
\text { mostly dolomite }\end{array}$ \\
\hline 2 & & $7.77-8.23$ & & & \\
\hline 2 & & $8.23-8.69$ & & & \\
\hline 2 & & $8.69-9.14$ & & & \\
\hline 2 & $76 S 71$ & $9.14-9.60$ & & 61.0 & $\begin{array}{l}\text { gray clayey silt, moist; grading to } \\
\text { gray-brown clayey silt }\end{array}$ \\
\hline 2 & & $9.60-10.06$ & & & \\
\hline 2 & & $10.06-10.52$ & & & \\
\hline 2 & $76 S 72$ & $10.52-10.97$ & & 53.3 & $\begin{array}{l}\text { grayish brown clayey silt } \mathrm{w} / \text { pebbles, } \\
\text { mostly dolomite }\end{array}$ \\
\hline 2 & & $10.97-11.43$ & & & \\
\hline 3 & & $11.43-11.89$ & & & \\
\hline 3 & $76 S 73$ & $11.89-12.34$ & & 17.8 & $\begin{array}{l}\text { gray clayey silt } w / \text { pebbles, mostly } \\
\text { dolomite }\end{array}$ \\
\hline & & EOB $12.50 \mathrm{~m}$ & & & \\
\hline
\end{tabular}


Table 2.--Test-well construction data and lithologic description of cores at Plot M--Continued

\section{Test well 4}

\section{Completion date: April 7, 1976}

Location: 32 meters north-northwest of the northeast corner of Plot M, $26 \mathrm{~m}$ northnortheast of test well 2

Site and well construction data:

Altitude of land surface . . . . . . . . . $207.87 \mathrm{~m}$

Altitude of measuring point (top of casing). . . . . $207.93 \mathrm{~m}$

Depth to bottom of piezometer from land surface. . . $12.13 \mathrm{~m}$

Type of piezometer: $1.52 \mathrm{~m}$ perforated PVC pipe, $6.35 \mathrm{~cm}$ diameter

\begin{tabular}{|c|c|c|c|c|c|}
\hline $\begin{array}{l}\text { Unit } \\
\text { No. }\end{array}$ & $\begin{array}{c}\text { Sample } \\
\text { No. }\end{array}$ & $\begin{array}{c}\text { Depth } \\
\text { interval } \\
\text { (meters) } \\
\end{array}$ & $\begin{array}{l}\text { Blow } \\
\text { counts }\end{array}$ & $\begin{array}{l}\text { Recovery } \\
(\mathrm{cm})\end{array}$ & Description \\
\hline 1 & $76 S 52$ & $0-0.46$ & & 33.6 & brown silty clay $\mathrm{w} / \mathrm{stones,}$ root tubes \\
\hline 1 & 76553 & $0.46-0.91$ & & 33.0 & brown silty clay $w /$ numerous pebbles \\
\hline 1 & & $0.91-1.37$ & & & \\
\hline 1 & 76554 & $1.37-1.83$ & & 25.4 & brown clayey silt w/pebbles \\
\hline 1 & & $1.83-2.29$ & & & \\
\hline 1 & 76555 & $2.29-2.74$ & & 19.1 & brown clayey silt; vertical fracture \\
\hline 1 & $76 S 56$ & $2.74-3.20$ & & 27.9 & $\begin{array}{l}\text { brown clayey silt and sand to } 3.08 \mathrm{~m} \text {; } \\
\text { contact } \mathrm{w} / 27.9 \mathrm{~cm} \text { water-bearing sand } \\
\mathrm{w} / \mathrm{small} \text { pebbles; contact } \mathrm{w} / \mathrm{brown} \\
\text { clayey silt } \mathrm{w} / \text { pebbles }\end{array}$ \\
\hline
\end{tabular}

\begin{tabular}{|c|c|c|c|c|}
\hline 1 & & $3.20-3.66$ & & \\
\hline 1 & & $3.66-4.12$ & & \\
\hline 1 & & $4.12-4.57$ & & \\
\hline 2 & & $4.57-5.03$ & & \\
\hline 2 & 76557 & $5.03-5.49$ & 19.1 & $\begin{array}{l}\text { gray silty clay grading to brown clay } \\
\text { to } 5.18 \mathrm{~m} \text {; contact } \mathrm{w} / 15.2 \mathrm{~cm} \text { brown } \\
\text { silty sand at } 5.18 \mathrm{~m}\end{array}$ \\
\hline
\end{tabular}


Table 2.--Test-well construction data and lithologic description of cores at Plot M--Continued

Test well 4--Continued

\begin{tabular}{|c|c|c|c|c|c|}
\hline $\begin{array}{l}\text { Unit } \\
\text { No. }\end{array}$ & $\begin{array}{l}\text { Sample } \\
\text { No. }\end{array}$ & $\begin{array}{c}\text { Depth } \\
\text { interval } \\
\text { (meters) }\end{array}$ & $\begin{array}{l}\text { Blow } \\
\text { counts }\end{array}$ & $\begin{array}{l}\text { Recovery } \\
\quad(\mathrm{cm})\end{array}$ & Description \\
\hline 2 & & $5.49-5.94$ & & & \\
\hline 2 & 76558 & $5.94-6.40$ & & 29.2 & gray clayey silt $\mathrm{w} / \mathrm{limestone}$ pebbles \\
\hline 2 & & $6.40-6.86$ & & & \\
\hline 2 & & $6.86-7.32$ & & & \\
\hline 2 & $76 \$ 59$ & $7.32-7.77$ & & 48.3 & $\begin{array}{l}\text { brown-gray clayey silt; contact } \\
\text { w/gray-brown clayey silt }\end{array}$ \\
\hline 2 & & $7.77-8.23$ & & & \\
\hline 2 & & $8.23-8.69$ & & & \\
\hline 2 & & $8.69-9.14$ & & & \\
\hline 2 & $76 S 60$ & $9.14-9.60$ & & 22.9 & $\begin{array}{l}\text { gray-brown clayey silt } \mathrm{w} / \text { numerous } \\
\text { pebbles }\end{array}$ \\
\hline 2 & & $9.60-10.06$ & & & \\
\hline 2 & & $10.06-10.52$ & & & \\
\hline 2 & 76561 & $10.52-10.97$ & & 61.0 & $\begin{array}{l}\text { gray clayey silt w/pebbles, mostly } \\
\text { dolomite }\end{array}$ \\
\hline 2 & & $10.97-11.43$ & & & \\
\hline 2 & & $11.43-11.89$ & & & \\
\hline 2 & $76 \$ 62$ & $11.89-12.34$ & & 30.5 & $\begin{array}{l}\text { gray clayey silt } w / \text { pebbles, moist to } \\
\text { wet }\end{array}$ \\
\hline & & EOB $12.50 \mathrm{~m}$ & & & \\
\hline
\end{tabular}


Table 2.--Test-well construction data and lithologic description of cores at Plot M--Continued

Test well 5

Completion date: April 8, 1976

Location: 60 meters north of the northeast corner of Plot $M$

Site and well construction data:

Altitude of land surface . . . . . . . . . . $204.89 \mathrm{~m}$

Altitude of measuring point (top of casing). . . . . $205.06 \mathrm{~m}$

Depth to bottom of piezometer from land surface. . . $12.02 \mathrm{~m}$

Type of piezometer: $1.52 \mathrm{~m}$ perforated PVC pipe, $6.35 \mathrm{~cm}$ diameter

\begin{tabular}{|c|c|c|c|c|c|}
\hline $\begin{array}{l}\text { Unit } \\
\text { No. }\end{array}$ & $\begin{array}{l}\text { Sample } \\
\text { No. }\end{array}$ & $\begin{array}{c}\text { Depth } \\
\text { interval } \\
\text { (meters) } \\
\end{array}$ & $\begin{array}{l}\text { Blow } \\
\text { counts }\end{array}$ & $\begin{array}{l}\text { Recovery } \\
\quad(\mathrm{cm})\end{array}$ & Description \\
\hline 1 & $76 \mathrm{~S} 85$ & $0-0.46$ & & 20.3 & $\begin{array}{l}\text { yellow-brown silty clay } w / \text { plant } \\
\text { fragments, tree roots }\end{array}$ \\
\hline 1 & $76 S 86$ & $0.46-0.91$ & & 21.6 & brown silty clay w/tree roots \\
\hline 1 & 76587 & $0.91-1.37$ & & 16.5 & brown silty clay w/stones \\
\hline 1 & & $1.37-1.83$ & & & \\
\hline 1 & $76 S 88$ & $1.83-2.29$ & & 21.6 & brown silty clay w/stones \\
\hline 1 & & $2.29-2.74$ & & & \\
\hline 1 & 76589 & $2.74-3.20$ & & 36.8 & $\begin{array}{l}\text { brown-gray silty clay } w / \text { limestone } \\
\text { pebbles; grading to brown clayey silt; } \\
\text { grading to gray clayey silt w/pebbles }\end{array}$ \\
\hline 1 & & $3.20-3.66$ & & & \\
\hline 2 & & $3.66-4.12$ & & & \\
\hline 2 & & $4.12-4.57$ & & & \\
\hline 2 & 76590 & $4.57-5.03$ & & 39.4 & $\begin{array}{l}\text { gray clayey silt } w / \text { pebbles, mostly } \\
\text { dolomite }\end{array}$ \\
\hline 2 & & $5.03-5.49$ & & & \\
\hline 2 & & $5.49-5.94$ & & & \\
\hline
\end{tabular}


Table 2.--Test-well construction data and lithologic description of cores at Plot M--Continued

Test well 5--Continued

\begin{tabular}{|c|c|c|c|c|c|}
\hline $\begin{array}{l}\text { Unit } \\
\text { No. }\end{array}$ & $\begin{array}{c}\text { Sample } \\
\text { No. }\end{array}$ & $\begin{array}{l}\text { Depth } \\
\text { interval } \\
\text { recovery }\end{array}$ & $\begin{array}{l}\text { Blow } \\
\text { counts }\end{array}$ & $\begin{array}{l}\text { Recovery } \\
\quad(\mathrm{cm})\end{array}$ & Description \\
\hline 2 & $76 S 91$ & $5.94-6.40$ & & 20.3 & $\begin{array}{l}\text { gray clayey silt w/pebbles, stones, } \\
\text { mostly dolomite }\end{array}$ \\
\hline 2 & & $6.40-6.86$ & & & \\
\hline 2 & & $6.86-7.32$ & & & \\
\hline 2 & 76592 & $7.32-7.77$ & & 48.3 & $\begin{array}{l}\text { gray clayey silt to } 7.70 \mathrm{~m} ; 5.1 \mathrm{~cm} \\
\text { sand layer at } 7.77 \mathrm{~m} ; \text { contact w/gray- } \\
\text { brown clayey silt } \mathrm{w} / \text { numerous pebbles }\end{array}$ \\
\hline 2 & & $7.77-8.23$ & & & \\
\hline 2 & & $8.23-8.69$ & & & \\
\hline 2 & & $8.69-9.14$ & & & \\
\hline 2 & 76593 & $9.14-9.60$ & & 39.4 & $\begin{array}{l}\text { gray clayey silt; grading to brown- } \\
\text { gray clayey silt w/pebbles, stones, } \\
\text { mostly dolomite }\end{array}$ \\
\hline 2 & & $9.60-10.06$ & & & \\
\hline 2 & & $10.06-10.52$ & & & \\
\hline 2 & 76594 & $10.52-10.97$ & & 31.8 & $\begin{array}{l}\text { gray clayey silt w/few pebbles; grad- } \\
\text { ing to brownish-gray clayey silt } \\
\text { w/numerous large stones and pebbles }\end{array}$ \\
\hline 2 & & $10.97-11.43$ & & & \\
\hline 2 & & $11.43-11.89$ & & & \\
\hline 2 & $76 s 95$ & $11.89-12.19$ & & 16.5 & gray clayey silt w/pebbles \\
\hline
\end{tabular}


Table 2.--Test-well construction data and lithologic description of cores at Plot M--Continued

\section{Test well 6}

Completion date: April 5, 1976

Location: 30 meters south of the northeast corner of Plot M, 18 meters northeast of the southeast corner

Site and well construction data:

Altitude of land surface . . . . . . . . . . $214.64 \mathrm{~m}$

Altitude of measuring point (top of casing). . . . . $214.84 \mathrm{~m}$

Depth of bottom of piezometer from land surface. . . . $11.99 \mathrm{~m}$

Type of piezometer: $1.52 \mathrm{~m}$ perforated PVC pipe, $6.35 \mathrm{~cm}$ diameter

\begin{tabular}{|c|c|c|c|c|c|}
\hline $\begin{array}{l}\text { Unit } \\
\text { No. }\end{array}$ & $\begin{array}{l}\text { Sample } \\
\text { No. }\end{array}$ & $\begin{array}{c}\text { Depth } \\
\text { interval } \\
\text { (meters) }\end{array}$ & $\begin{array}{l}\text { Blow } \\
\text { counts }\end{array}$ & $\begin{array}{l}\text { Recovery } \\
\quad(\mathrm{cm})\end{array}$ & Description \\
\hline 1 & $76 \mathrm{~S} 30$ & $0-0.46$ & & 25.4 & brown silty clay $\mathrm{w} / \mathrm{stones,}$ root tubes \\
\hline 1 & & $0.46-0.91$ & & & \\
\hline 1 & $76 S 31$ & $0.91-1.37$ & & 33.0 & brown silty clay $\mathrm{w} / \mathrm{stones,} \mathrm{moist}$ \\
\hline 1 & 76532 & $1.37-1.83$ & & 32.5 & $\begin{array}{l}\text { brown silty clay } \mathrm{w} / \mathrm{stones,} \mathrm{mottled;} \\
\text { sand parting at } 1.79 \mathrm{~m}\end{array}$ \\
\hline 1 & & $1.83-2.29$ & & & \\
\hline 1 & 76533 & $2.29-2.74$ & & 25.9 & $\begin{array}{l}\text { brown silty clay w/pebbles, stones, } \\
\text { mottled }\end{array}$ \\
\hline 1 & $76 \mathrm{~S} 34$ & $2.74-3.20$ & & 32.4 & brown silty clay w/pebbles, stones \\
\hline 1 & & $3.20-3.66$ & & & \\
\hline 1 & & $3.66-4.12$ & & & \\
\hline 1 & & $4.12-4.57$ & & & \\
\hline 1 & $76 \mathrm{~S} 35$ & $4.57-5.03$ & & 13.9 & $\begin{array}{l}\text { brown silty clay } w / \text { pebbles, large } \\
\text { variety of rocks and minerals }\end{array}$ \\
\hline 1 & & $5.03-5.49$ & & & \\
\hline 2 & & $5.49-5.94$ & & & \\
\hline
\end{tabular}


Table 2.--Test-well construction data and lithologic description of cores at Plot M--Continued

Test well 6--Continued

\begin{tabular}{|c|c|c|c|c|c|}
\hline $\begin{array}{l}\text { Unit } \\
\text { No. }\end{array}$ & $\begin{array}{l}\text { Sample } \\
\text { No. }\end{array}$ & $\begin{array}{l}\text { Depth } \\
\text { interval } \\
\text { (meters) }\end{array}$ & $\begin{array}{l}\text { Blow } \\
\text { counts }\end{array}$ & $\begin{array}{l}\text { Recovery } \\
\quad(\mathrm{cm})\end{array}$ & Description \\
\hline 2 & $76 \$ 36$ & $5.94-6.40$ & & 44.0 & $\begin{array}{l}\text { gray clayey silt } w / \text { pebbles, stones, } \\
\text { mostly dolomite }\end{array}$ \\
\hline 2 & & $6.40-6.86$ & & & \\
\hline 2 & & $6.86-7.32$ & & & \\
\hline 2 & $76 \mathrm{~S} 37$ & $7.32-7.77$ & & 22.2 & $\begin{array}{l}\text { gray clayey silt w/pebbles, stones, } \\
\text { mostly dolomite }\end{array}$ \\
\hline 2 & & $7.77-8.23$ & & & \\
\hline 2 & & $8.23-8.69$ & & & \\
\hline 2 & & $8.69-9.14$ & & & \\
\hline 2 & 76538 & $9.14-9.60$ & & 12.7 & gray clayey silt $\mathrm{w} /$ stones \\
\hline 2 & & $9.60-10.06$ & & & \\
\hline 2 & & $10.06-10.52$ & & & \\
\hline 2 & 76539 & $10.52-10.97$ & & 21.6 & $\begin{array}{l}\text { gray clayey silt } w / \text { pebbles, stones; } \\
\text { sand parting at } 10.75 \mathrm{~m}\end{array}$ \\
\hline 2 & & $10.97-11.43$ & & & \\
\hline 2 & & $11.43-11.89$ & & & \\
\hline 2 & & $11.89-12.34$ & & & \\
\hline 2 & $76 S 40$ & $12.34-12.65$ & & 27.9 & $\begin{array}{l}\text { gray clayey silt w/pebbles, stones, } \\
\text { mostly dolomite }\end{array}$ \\
\hline
\end{tabular}

EOB $12.65 \mathrm{~m}$ 
Table 2.--Test-well construction data and lithologic description of cores at Plot M--Continued

Test well 7

Completion date: April 2, 1976

Location: 38 meters southeast of the center of Plot M

Site and well construction data:

Altitude of land surface . . . . . . . . $216.26 \mathrm{~m}$

Altitude of measuring point (top of casing). . . . . $216.40 \mathrm{~m}$

Depth to bottom of piezometer from land surface. . . $12.05 \mathrm{~m}$

Type of piezometer: $1.52 \mathrm{~m}$ perforated PVC pipe, $6.35 \mathrm{~cm}$ diameter

\begin{tabular}{|c|c|c|c|c|c|}
\hline $\begin{array}{l}\text { Unit } \\
\text { No. }\end{array}$ & $\begin{array}{c}\text { Sample } \\
\text { No. }\end{array}$ & $\begin{array}{c}\text { Depth } \\
\text { interval } \\
\text { (meters) }\end{array}$ & $\begin{array}{l}\text { Blow } \\
\text { counts }\end{array}$ & $\begin{array}{l}\text { Recovery } \\
\quad(\mathrm{cm})\end{array}$ & Description \\
\hline 1 & $76 S 19$ & $0-0.46$ & & 17.8 & brown silty clay \\
\hline 1 & $76 S 20$ & $0.46-0.91$ & & 22.9 & brown silty clay w/pebbles, stones \\
\hline 1 & & $0.91-1.37$ & & & \\
\hline 1 & $76 S 21$ & $1.37-1.83$ & & 15.2 & brown silty clay w/pebbles, stones \\
\hline 1 & & $1.83-2.29$ & & & \\
\hline 1 & $76 S 22$ & $2.29-2.74$ & & 30.5 & brown silty clay w/pebbles, stones \\
\hline 1 & & $2.74-3.20$ & & & \\
\hline 1 & $76 S 23$ & $3.20-3.66$ & & 33.0 & $\begin{array}{l}\text { brown clayey silt w/pebbles, stones, } \\
\text { sandy }\end{array}$ \\
\hline 1 & & $3.66-4.12$ & & & \\
\hline 1 & & $4.12-4.57$ & & & \\
\hline 1 & $76 S 24$ & $4.57-5.03$ & & 40.6 & brown clayey silt w/pebbles, sandy \\
\hline 1 & & $5.03-5.49$ & & & \\
\hline 1 & & $5.49-5.94$ & & & \\
\hline 1 & $76 S 25$ & $5.94-6.40$ & & 43.2 & brownish gray clayey silt \\
\hline 2 & & $6.40-6.86$ & & & \\
\hline
\end{tabular}


Table 2.--Test-well construction data and lithologic description of cores at Plot M--Continued

Test well 7--Continued

\begin{tabular}{|c|c|c|c|c|c|}
\hline $\begin{array}{l}\text { Unit } \\
\text { No. }\end{array}$ & $\begin{array}{l}\text { Sample } \\
\text { No. }\end{array}$ & $\begin{array}{l}\text { Depth } \\
\text { interval } \\
\text { (meters) }\end{array}$ & $\begin{array}{l}\text { Blow } \\
\text { counts }\end{array}$ & $\begin{array}{l}\text { Recovery } \\
\quad(\mathrm{cm})\end{array}$ & Description \\
\hline 2 & & $6.86-7.32$ & & & \\
\hline 2 & & $7.32-7.77$ & & & \\
\hline 2 & $76 s 26$ & $7.77-8.23$ & & 35.6 & $\begin{array}{l}\text { gray clayey silt w/pebbles, mostly } \\
\text { dolomite }\end{array}$ \\
\hline 2 & & $8.23-8.69$ & & & \\
\hline 2 & & $8.69-9.14$ & & & \\
\hline 2 & $76 S 27$ & $9.14-9.60$ & & 45.7 & $\begin{array}{l}\text { dark gray clayey silt } w / \text { pebbles, } \\
\text { mostly dolomite }\end{array}$ \\
\hline 2 & & $9.60-10.06$ & & & \\
\hline 2 & & $10.06-10.52$ & & & \\
\hline 2 & $76 S 28$ & $10.52-10.97$ & & -- & $\begin{array}{l}\text { gray clayey silt w/pebbles, sand, } \\
\text { gravel }\end{array}$ \\
\hline 2 & & $10.97-11.43$ & & & \\
\hline 2 & & $11.43-11.89$ & & & \\
\hline 2 & & $11.89-12.34$ & & & \\
\hline 2 & $76 \$ 29$ & $12.34-12.65$ & & -- & $\begin{array}{l}\text { gray clayey silt w/pebbles, mostly } \\
\text { dolomite }\end{array}$ \\
\hline
\end{tabular}

EOB $12.65 \mathrm{~m}$ 
Table 2.--Test-well construction data and lithologic description of cores at Plot M--Continued

Test well 8

Completion date: April 8, 1976

Location: 67 meters southwest of test well 7

Site and well construction data:

Altitude of land surface. . . . . . . . . . $222.80 \mathrm{~m}$

Altitude of measuring point (top of casing). . . . $222.88 \mathrm{~m}$

Depth of bottom of piezometer from land surface. . . $12.11 \mathrm{~m}$

Type of piezometer: $1.52 \mathrm{~m}$ perforated PVC pipe, $6.35 \mathrm{~cm}$ diameter

\begin{tabular}{|c|c|c|c|c|c|}
\hline $\begin{array}{l}\text { Unit } \\
\text { No. }\end{array}$ & $\begin{array}{l}\text { Sample } \\
\text { No. }\end{array}$ & $\begin{array}{c}\text { Depth } \\
\text { interval } \\
\text { (meters) }\end{array}$ & $\begin{array}{l}\text { Blow } \\
\text { counts }\end{array}$ & $\begin{array}{l}\text { Recovery } \\
\quad(\mathrm{cm})\end{array}$ & Description \\
\hline 1 & $76 S 74$ & $0-0.46$ & & 36.8 & $\begin{array}{l}\text { yellow-brown silty clay w/pebbles, } \\
\text { root tubes }\end{array}$ \\
\hline 1 & $76 S 75$ & $0.46-0.91$ & & 23.5 & brown silty clay $w /$ few pebbles \\
\hline 1 & 76576 & $0.91-1.37$ & & 21.6 & brown silty clay w/pebbles; mottled \\
\hline 1 & & $1.37-1.83$ & & & \\
\hline 1 & $76 S 77$ & $1.83-2.29$ & & 21.6 & brown clayey silt, mottled \\
\hline 1 & & $2.29-2.74$ & & & \\
\hline 1 & & $2.74-3.20$ & & & \\
\hline 1 & $76 S 78$ & $3.20-3.66$ & & 21.6 & $\begin{array}{l}\text { brown clayey silt w/pebbles, mottled; } \\
5.1 \mathrm{~cm} \text { sand parting at } 3.40 \mathrm{~m}\end{array}$ \\
\hline 1 & & $3.66-4.12$ & & & \\
\hline 1 & & $4.12-4.57$ & & & \\
\hline 1 & $76 S 79$ & $4.57-5.03$ & & 20.3 & $\begin{array}{l}\text { brown clayey silt w/pebbles; } 45^{\circ} \\
\text { fracture, } 30.5 \mathrm{~cm} \text { in length }\end{array}$ \\
\hline 1 & & $5.03-5.49$ & & & \\
\hline 1 & & $5.49-5.94$ & & & \\
\hline
\end{tabular}


Table 2.--Test-well construction data and lithologic description of cores at Plot M--Continued

Test well 8--Continued

\begin{tabular}{|c|c|c|c|c|c|}
\hline $\begin{array}{l}\text { Unit } \\
\text { No. }\end{array}$ & $\begin{array}{l}\text { Sample } \\
\text { No. }\end{array}$ & $\begin{array}{c}\text { Depth } \\
\text { interval } \\
\text { (meters) }\end{array}$ & $\begin{array}{l}\text { Blow } \\
\text { counts }\end{array}$ & $\begin{array}{l}\text { Recovery } \\
(\mathrm{cm})\end{array}$ & Description \\
\hline 2 & $76 S 80$ & $5.94-6.40$ & & 43.2 & $\begin{array}{l}\text { brown clayey silt } w / \text { few pebbles; } \\
\text { vertical fractures }\end{array}$ \\
\hline 2 & & $6.40-6.86$ & & & \\
\hline 2 & & $6.86-7.32$ & & & \\
\hline 2 & $76 S 81$ & $7.32-7.77$ & & 34.3 & $\begin{array}{l}\text { brown clayey silt; } 45^{\circ} \text { fracture, } \\
30.5 \mathrm{~cm} \text { in length }\end{array}$ \\
\hline 2 & & $7.77-8.23$ & & & \\
\hline 2 & & $8.23-8.69$ & & & \\
\hline 2 & & $8.69-9.14$ & & & \\
\hline 2 & $76 s 82$ & $9.14-9.60$ & & 45.1 & $\begin{array}{l}\text { brown clayey silt } \mathrm{w} / \text { pebbles to } 9.26 \mathrm{~m} \text {; } \\
\text { then gray clayey silt w/limestone } \\
\text { pebbles }\end{array}$ \\
\hline 2 & & $9.60-10.06$ & & & \\
\hline 2 & & $10.06-10.52$ & & & \\
\hline 2 & $76 S 83$ & $10.52-10.97$ & & -- & brown clayey silt \\
\hline 2 & & $10.97-11.43$ & & & \\
\hline 2 & & $11.43-11.89$ & & & \\
\hline 2 & 76584 & $11.89-12.34$ & & -- & gray clayey silt, moist \\
\hline
\end{tabular}


Table 2.--Test-well construction data and lithologic description of cores at Plot M--Continued

Test well 9

Completion date: April 28, 1976

Location: Northeast corner of Plot $M$

Site and well construction data: Slant borehole, $45^{\circ}$ angle towards northeast beneath $\mathrm{P}$ lot $M$

Altitude of land surface ............. $212.17 \mathrm{~m}$

Altitude of measuring point (top of casing). . . . . $212.17 \mathrm{~m}$

Horizontal distance from measuring point . . . . . $12.93 \mathrm{~m}$

Depth of bottom of piezometer from land surface. . . . $13.90 \mathrm{~m}$

Type of piezometer: $1.52 \mathrm{~m}$ perforated PVC pipe, $6.35 \mathrm{~cm}$ diameter

\begin{tabular}{|c|c|c|c|c|c|}
\hline $\begin{array}{l}\text { Unit } \\
\text { No. }\end{array}$ & $\begin{array}{l}\text { Sample } \\
\text { No. }\end{array}$ & $\begin{array}{c}\text { Depth } \\
\text { interval } \\
\text { (meters) }\end{array}$ & $\begin{array}{l}\text { Blow } \\
\text { counts }\end{array}$ & $\begin{array}{l}\text { Recovery } \\
\quad(\mathrm{cm})\end{array}$ & Description \\
\hline 1 & 765111 & $0-0.46$ & & 25.4 & brown silty clay \\
\hline 1 & & $0.46-0.91$ & & & \\
\hline 1 & $76 S 112$ & $0.91-1.37$ & & 28.6 & brown silty clay \\
\hline 1 & $76 S 113$ & $1.37-1.83$ & & 24.6 & brown silty clay w/pebbles, stones \\
\hline 1 & $76 S 114$ & $1.83-2.29$ & & 22.2 & $\begin{array}{l}0.63 \mathrm{~cm} \text { sand parting at } 2.10 \mathrm{~m} \text {; } \\
\text { brown silty clay } \mathrm{w} / \text { pebbles, stones }\end{array}$ \\
\hline 1 & & $2.29-2.74$ & & & \\
\hline 1 & & $2.74-3.20$ & & & \\
\hline 1 & $76 S 115$ & $3.20-3.66$ & & 27.3 & brown silty clay w/pebbles, stones \\
\hline 1 & & $3.66-4.12$ & & & \\
\hline 1 & $76 S 116$ & $4.12-4.57$ & & 24.1 & $\begin{array}{l}\text { brown clayey silt w/stones; } 7.6 \mathrm{~cm} \\
\text { sand layer at } 4.50 \mathrm{~m}\end{array}$ \\
\hline 1 & & $4.57-5.03$ & & & \\
\hline 1 & $76 S 117$ & $5.03-5.49$ & & 39.4 & $\begin{array}{l}\text { brown clayey silt w/numerous pebbles, } \\
\text { moist to } 5.40 \mathrm{~m} \text {; contact w/gray-brown } \\
\text { clayey silt } \mathrm{w} / \mathrm{few} \text { pebbles grading to } \\
\text { gray clayey silt }\end{array}$ \\
\hline
\end{tabular}


Table 2.--Test-well construction data and lithologic description of cores at Plot M--Continued

\section{Test well 9--Continued}

\begin{tabular}{|c|c|c|c|c|c|}
\hline $\begin{array}{l}\text { Unit } \\
\text { No. }\end{array}$ & $\begin{array}{l}\text { Sample } \\
\text { No. }\end{array}$ & $\begin{array}{c}\text { Depth } \\
\text { interval } \\
\text { (meters) }\end{array}$ & $\begin{array}{l}\text { Blow } \\
\text { counts }\end{array}$ & $\begin{array}{l}\text { Recovery } \\
\quad(\mathrm{cm})\end{array}$ & Description \\
\hline 1 & & $5.49-5.94$ & & & \\
\hline 1 & $76 S 118$ & $5.94-6.40$ & & 24.1 & brown clayey silt $w /$ pebbles, moist \\
\hline 2 & & $6.40-6.96$ & & & \\
\hline 2 & $76 S 119$ & $6.96-7.32$ & & 29.2 & $\begin{array}{l}\text { brownish gray clayey silt } \mathrm{w} / \text { numerous } \\
\text { pebbles, moist to wet; contact } \mathrm{w} / \mathrm{gray} \\
\text { clayey silt } \mathrm{w} / \text { pebbles }\end{array}$ \\
\hline 2 & & $7.32-7.77$ & & & \\
\hline 2 & & $7.77-8.23$ & & & \\
\hline 2 & $76 S 120$ & $8.23-8.69$ & & 38.1 & $\begin{array}{l}\text { gray clayey silt w/pebbles, moist to } \\
\text { wet, mostly dolomite grains }\end{array}$ \\
\hline 2 & & $8.69-9.14$ & & & \\
\hline 2 & $76 S 121$ & $9.14-9.60$ & & 12.7 & $\begin{array}{l}\text { gray clayey silt w/stones, mottled, } \\
\text { moist }\end{array}$ \\
\hline 2 & & $9.60-10.06$ & & & \\
\hline 2 & & $10.06-10.52$ & & & \\
\hline 2 & $76 S 122$ & $10.52-10.97$ & & 37.5 & $\begin{array}{l}\text { gray clayey silt w/pebbles, mostly } \\
\text { dolomite }\end{array}$ \\
\hline 2 & & $10.97-11.43$ & & & \\
\hline 2 & $76 S 123$ & $11.43-11.89$ & & 16.5 & brown silty sand, moist \\
\hline 2 & & $11.89-12.34$ & & & \\
\hline 2 & & $12.34-13.11$ & & & \\
\hline 2 & $76 S 124$ & $13.11-13.15$ & & 24.1 & gray-brown clayey silt \\
\hline & & EOB $13.15 \mathrm{~m}$ & & & \\
\hline
\end{tabular}


Table 2.--Test-well construction data and lithologic description of cores at Plot M--Continued

Test well 10

Completion date: April 26, 1976

Location: Southwest corner of Plot $M$

Site and well construction data: Slant borehole, $45^{\circ}$ angle towards northeast beneath $P$ lot $M$

Altitude of land surface . . . . . . . . . $216.34 \mathrm{~m}$

Altitude of measuring point (top of casing). . . . . $216.34 \mathrm{~m}$

Horizontal distance from measuring point....... $12.93 \mathrm{~m}$

Depth of bottom of piezometer from land surface. . . . $12.50 \mathrm{~m}$

Type of piezometer: $1.56 \mathrm{~m}$ perforated PVC pipe, $6.35 \mathrm{~cm}$ diameter

\begin{tabular}{|c|c|c|c|c|c|}
\hline $\begin{array}{l}\text { Unit } \\
\text { No. }\end{array}$ & $\begin{array}{l}\text { Sample } \\
\text { No. }\end{array}$ & $\begin{array}{c}\text { Depth } \\
\text { interval } \\
\text { (meters) }\end{array}$ & $\begin{array}{l}\text { Blow } \\
\text { counts }\end{array}$ & $\begin{array}{l}\text { Recovery } \\
(\mathrm{cm})\end{array}$ & Description \\
\hline 1 & $76 \mathrm{~S} 96$ & $0-0.46$ & & 20.3 & brown silty clay \\
\hline 1 & $76 S 97$ & $0.46-0.91$ & & 29.2 & $\begin{array}{l}\text { brown silty clay to } 0.91 \mathrm{~m} \text {; contact } \\
\text { w/dark brown silty clay }\end{array}$ \\
\hline 1 & $76 S 98$ & $0.91-1.37$ & & 29.2 & brown silty clay \\
\hline 1 & $76 S 99$ & $1.37-1.83$ & & 21.0 & brown silty clay, mottled \\
\hline 1 & $76 S 100$ & $1.83-2.29$ & & 19.1 & brown silty clay, mottled \\
\hline 1 & & $2.29-2.74$ & & & \\
\hline 1 & & $2.74-3.20$ & & & \\
\hline 1 & $76 S 101$ & $3.20-3.66$ & & 33.7 & brown silty clay $w /$ pebbles \\
\hline 1 & & $3.66-4.12$ & & & \\
\hline 1 & $76 S 102$ & $4.12-4.57$ & & 35.6 & brown clayey silt w/pebbles \\
\hline 1 & & $4.57-5.03$ & & & \\
\hline 1 & $76 S 103$ & $5.03-5.49$ & & 42.5 & brown clayey silt \\
\hline 1 & & $5.49-5.94$ & & & \\
\hline
\end{tabular}


Table 2.--Test-well construction data and lithologic description of cores at Plot M--Continued

Test well 10--Continued

\begin{tabular}{|c|c|c|c|c|c|}
\hline $\begin{array}{l}\text { Unit } \\
\text { No. }\end{array}$ & $\begin{array}{l}\text { Sample } \\
\text { No. }\end{array}$ & $\begin{array}{c}\text { Depth } \\
\text { interval } \\
\text { (meters) }\end{array}$ & $\begin{array}{l}\text { Blow } \\
\text { counts }\end{array}$ & $\begin{array}{l}\text { Recovery } \\
\quad(\mathrm{cm})\end{array}$ & Description \\
\hline 1 & 765104 & $5.94-6.40$ & & 47.6 & $\begin{array}{l}\text { brown clayey silt w/pebbles to } 6.71 \mathrm{~m} \text {; } \\
\text { contact w/gray-brown clayey silt } \\
\text { w/pebbles, mostly dolomite }\end{array}$ \\
\hline 2 & & $6.40-6.96$ & & & \\
\hline 2 & $76 S 1.05$ & $6.96-7.32$ & & 31.8 & $\begin{array}{l}\text { brown clayey silt w/pebbles to } 7.62 \mathrm{~m} \text {; } \\
\text { contact w/gray-brown clayey silt }\end{array}$ \\
\hline 2 & & $7.32-7.77$ & & & \\
\hline 2 & & $7.77-8.23$ & & & \\
\hline 2 & $76 S 106$ & $8.23-8.69$ & & 33.0 & $\begin{array}{l}\text { gray clayey silt w/pebbles, mostly } \\
\text { dolomite }\end{array}$ \\
\hline 2 & & $8.69-9.14$ & & & \\
\hline 2 & $76 \$ 107$ & $9.14-9.60$ & & 26.6 & $\begin{array}{l}\text { gray-brown clayey silt to } 9.75 \mathrm{~m} \text {; } \\
\text { contact } \mathrm{w} / \text { brown-gray silt } \mathrm{w} / \text { pebbles }\end{array}$ \\
\hline 2 & & $9.60-10.06$ & & & \\
\hline 2 & & $10.06-10.52$ & & & \\
\hline 2 & $76 \$ 108$ & $10.52-10.97$ & & 17.1 & brown-gray clayey silt w/pebbles \\
\hline 2 & & $10.97-11.43$ & & & \\
\hline 2 & $76 \$ 109$ & $11.43-11.89$ & & 14.6 & $\begin{array}{l}\text { gray clayey silt w/pebbles, stones, } \\
\text { moist }\end{array}$ \\
\hline 2 & & $11.89-12.34$ & & & \\
\hline 2 & & $12.34-13.11$ & & & \\
\hline 2 & $76 S 110$ & $13.11-13.15$ & & 27.9 & $\begin{array}{l}\text { gray clayey silt w/pebbles, stones, } \\
\text { moist }\end{array}$ \\
\hline
\end{tabular}

EOB $13.15 \mathrm{~m}$ 
Table 2.--Test-well construction data and lithologic description of cores at Plot M--Continued

Test well 11

Completion date: September 7, 1977

Location: Northeast corner of Plot $M$, next to test well 3

Site and well construction data: 3 test wells; a $37.80 \mathrm{~m}$ and a $20.73 \mathrm{~m}$ well nest in one borehole, and an adjacent $11.89 \mathrm{~m}$ test well

Altitude of land surface: $11.89 \mathrm{~m}$ test well. . . . . . . $211.14 \mathrm{~m}$

$20.73 \mathrm{~m}$ test well. . . . . . . $211.14 \mathrm{~m}$

$37.80 \mathrm{~m}$ test well. . . ...... $211.14 \mathrm{~m}$

Altitude of measuring point (top of casing): $11.89 \mathrm{~m}$ test well. $.211 .21 \mathrm{~m}$

$20.73 \mathrm{~m}$ test well. $211.25 \mathrm{~m}$

$37.80 \mathrm{~m}$ test well. . $211.25 \mathrm{~m}$

Depth of bottom of piezometer from land surface: $11.89 \mathrm{~m}$ test well. $.11 .82 \mathrm{~m}$ $20.73 \mathrm{~m}$ test well. $20.62 \mathrm{~m}$ $37.80 \mathrm{~m}$ test well. $37.69 \mathrm{~m}$

Type of piezometer: $1.52 \mathrm{~m}$ slotted PVC pipe, $5.08 \mathrm{~cm}$ diameter

\begin{tabular}{lccccc}
\hline $\begin{array}{l}\text { Unit } \\
\text { No. }\end{array}$ & $\begin{array}{c}\text { Sample } \\
\text { No. }\end{array}$ & $\begin{array}{c}\text { Depth } \\
\text { interval } \\
\text { (meters) }\end{array}$ & $\begin{array}{c}\text { Blow } \\
\text { counts }\end{array}$ & $\begin{array}{c}\text { Recovery } \\
(\mathrm{cm})\end{array}$ & Description \\
\hline 1 & 77587 & $0-0.46$ & 45.7 & brown silty clay w/pebbles, hard \\
\hline 1 & $0.46-0.91$ & & \\
\hline 1 & $0.91-1.37$ & 45.7 & not available \\
\hline 1 & 77588 & $1.37-1.83$ & & \\
\hline 1 & & & \\
\hline 1 & $1.83-2.29$ & & \\
\hline 1 & 77589 & $2.74-3.20$ & & \\
\hline 1 & $3.20-3.66$ & & \\
\hline 1 & $3.66-4.12$ & & \\
\hline 1 & $4.12-4.57$ & 45.7 & not available \\
\hline 1 & 77590 & $4.57-5.03$ & \\
\hline
\end{tabular}


Table 2.--Test-well construction data and lithologic description of cores at Plot M--Continued

Test well $11--$ Continued

\begin{tabular}{|c|c|c|c|c|c|}
\hline $\begin{array}{l}\text { Unit } \\
\text { No. }\end{array}$ & $\begin{array}{c}\text { Sample } \\
\text { No. }\end{array}$ & $\begin{array}{c}\text { Depth } \\
\text { interval } \\
\text { (meters) }\end{array}$ & $\begin{array}{l}\text { Blow } \\
\text { counts }\end{array}$ & $\begin{array}{l}\text { Recovery } \\
\quad(\mathrm{cm})\end{array}$ & Description \\
\hline 1 & & $5.03-5.49$ & & & \\
\hline 1 & & $5.49-5.94$ & & & \\
\hline 2 & $77 \mathrm{~S} 91$ & $5.94-6.40$ & & 45.7 & brown-gray clayey silt w/pebbles, hard \\
\hline 2 & & $6.40-6.96$ & & & \\
\hline 2 & & $6.96-7.32$ & & & \\
\hline 2 & 77592 & $7.32-7.77$ & & 45.7 & not available \\
\hline 2 & & $7.77-8.23$ & & & \\
\hline 2 & & $8.23-8.69$ & & & \\
\hline 2 & & $8.69-9.14$ & & & \\
\hline 2 & 77593 & $9.14-9.60$ & & 45.7 & not available \\
\hline 2 & & $9.60-10.06$ & & & \\
\hline 2 & & $10.06-10.52$ & & & \\
\hline 2 & 77594 & $10.52-10.97$ & & 45.7 & not available \\
\hline 2 & & $10.97-11.43$ & & & \\
\hline 3 & & $11.43-11.89$ & & & \\
\hline 3 & 77595 & $11.89-12.34$ & & 45.7 & not available \\
\hline 3 & & $12.34-13.11$ & & & \\
\hline 3 & & $13.11-13.56$ & & & \\
\hline 3 & 77S96 & $13.56-14.02$ & & 45.7 & not available \\
\hline 3 & & $14.02-14.48$ & & & \\
\hline
\end{tabular}


Table 2.--Test-well construction data and lithologic description of cores at Plot M--Continued

Test well 11--Continued

\begin{tabular}{|c|c|c|c|c|c|}
\hline $\begin{array}{l}\text { Unit } \\
\text { No. }\end{array}$ & $\begin{array}{l}\text { Sample } \\
\text { No. }\end{array}$ & $\begin{array}{c}\text { Depth } \\
\text { interval } \\
\text { (meters) }\end{array}$ & $\begin{array}{l}\text { Blow } \\
\text { counts }\end{array}$ & $\begin{array}{l}\text { Recovery } \\
\text { (cm) }\end{array}$ & Description \\
\hline 3 & & $14.48-14.94$ & & & \\
\hline 3 & 77597 & $14.94-15.39$ & & 45.7 & not available \\
\hline 3 & & $15.39-15.85$ & & & \\
\hline 3 & & $15.85-16.31$ & & & \\
\hline 3 & & $16.31-16.76$ & & & \\
\hline 3 & 77598 & $16.76-17.22$ & & 45.7 & not available \\
\hline 3 & & $17.22-17.68$ & & & \\
\hline 3 & & $17.68-18.14$ & & & \\
\hline 3 & 77599 & $18.14-18.59$ & & -- & $\begin{array}{l}\text { gray clayey silt w/pebbles, stones, } \\
\text { hard }\end{array}$ \\
\hline 3 & & $18.59-19.05$ & & & \\
\hline 3 & & $19.05-19.51$ & & & \\
\hline 4 & $77 \mathrm{~S} 100$ & $19.51-19.96$ & & 22.9 & not available \\
\hline 4 & & $19.96-20.42$ & & & \\
\hline 4 & & $20.42-20.88$ & & & \\
\hline 4 & & $20.88-21.33$ & & & \\
\hline 4 & 775101 & $21.33-21.79$ & & 25.4 & not available \\
\hline 4 & & $21.79-22.25$ & & & \\
\hline 4 & & $22.25-22.71$ & & & \\
\hline 4 & $77 S 102$ & $22.71-23.16$ & & 30.5 & not available \\
\hline
\end{tabular}


Table 2.--Test-well construction data and lithologic description of cores at Plot M--Continued

Test well 11--Continued

\begin{tabular}{|c|c|c|c|c|c|}
\hline $\begin{array}{l}\text { Unit } \\
\text { No. }\end{array}$ & $\begin{array}{c}\text { Sample } \\
\text { No. }\end{array}$ & $\begin{array}{c}\text { Depth } \\
\text { interval } \\
\text { (meters) }\end{array}$ & $\begin{array}{l}\text { Blow } \\
\text { counts }\end{array}$ & $\begin{array}{l}\text { Recovery } \\
\text { (cm) }\end{array}$ & Description \\
\hline 4 & & $23.16-23.62$ & & & \\
\hline 4 & & $23.62-24.08$ & & & \\
\hline 4 & 775103 & $24.08-24.53$ & & 30.5 & gray silt w/pebbles, stones, dense \\
\hline 5 & & $24.53-24.99$ & & & \\
\hline 5 & & $24.99-25.45$ & & & \\
\hline 5 & & $25.45-25.91$ & & & \\
\hline 5 & 775104 & $25.91-26.37$ & & 27.9 & not available \\
\hline 5 & & $26.37-26.82$ & & & \\
\hline 5 & & $26.82-27.28$ & & & \\
\hline 5 & $77 \mathrm{~S} 105$ & $27.28-27.74$ & & 12.7 & not available \\
\hline 5 & & $27.74-28.19$ & & & \\
\hline 5 & & $28.19-28.65$ & & & \\
\hline 5 & 775106 & $28.65-29.11$ & & 10.2 & gray silt, very dense \\
\hline 5 & & $29.11-29.57$ & & & \\
\hline 5 & & $29.57-30.02$ & & & \\
\hline 5 & & $30.02-30.48$ & & & \\
\hline 5 & 775107 & $30.48-30.94$ & & 15.2 & gray sandy silt, very dense \\
\hline 5 & & $30.94-31.39$ & & & \\
\hline 6 & & $31.39-31.85$ & & & \\
\hline 6 & 775108 & $31.85-32.32$ & & 45.7 & not available \\
\hline
\end{tabular}


Table 2.--Test-well construction data and lithologic description of cores at Plot $\mathrm{M}$--Continued

\section{Test well 11--Continued}

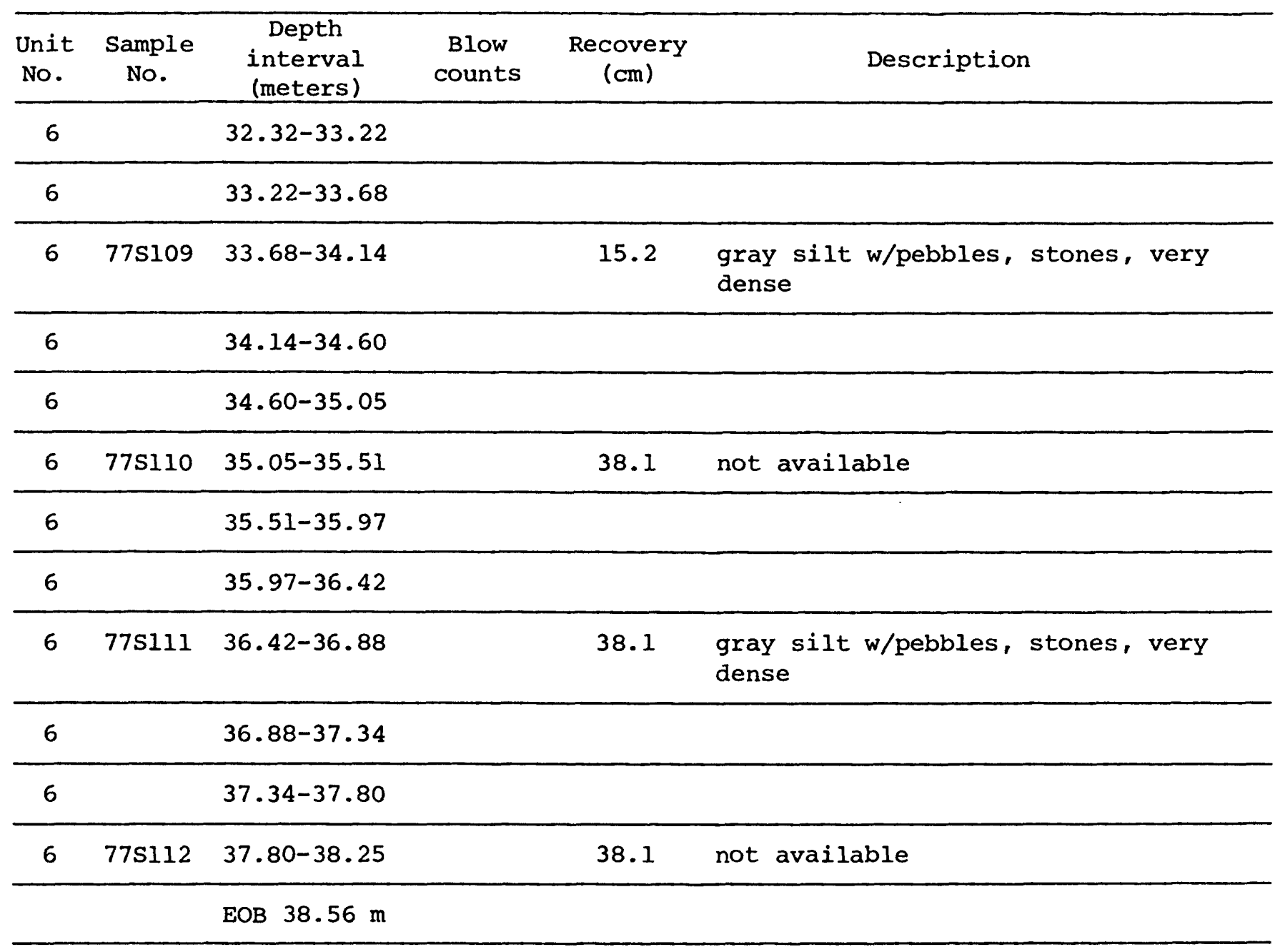


Table 2.--Test-well construction data and lithologic description of cores at Plot M--Continued

Test we11 21

Completion date: October 1, 1976

Location: 160 meters south of Plot $M$ on the south edge of the forest preserve road Site and well construction data:

Altitude of land surface . . . . . . . . . $221.13 \mathrm{~m}$

Altitude of measuring point (top of casing). . . . $221.20 \mathrm{~m}$

Depth to bottom of test well from land surface. . . $22.86 \mathrm{~m}$

Type of well: $2.54 \mathrm{~cm}$ diameter PVC pipe, no piezometer

\begin{tabular}{|c|c|c|c|c|c|}
\hline $\begin{array}{l}\text { Unit } \\
\text { No. }\end{array}$ & $\begin{array}{l}\text { Sample } \\
\text { No. }\end{array}$ & $\begin{array}{c}\text { Depth } \\
\text { interval } \\
\text { (meters) }\end{array}$ & $\begin{array}{l}\text { Blow } \\
\text { counits }\end{array}$ & $\begin{array}{l}\text { Recovery } \\
(\mathrm{cm})\end{array}$ & Description \\
\hline 1 & $76 S 291$ & $0-0.46$ & $2-4-4$ & 35.6 & brown silty clay \\
\hline 1 & & $0.46-0.91$ & & & \\
\hline 1 & & $0.91-1.37$ & & & \\
\hline 1 & $76 S 292$ & $1.37-1.83$ & $8-10-16$ & 45.7 & $\begin{array}{l}\text { brown silty clay, trace of sand, } \\
\text { gravel }\end{array}$ \\
\hline 1 & & $1.83-2.29$ & & & \\
\hline 1 & & $2.29-2.74$ & & & \\
\hline 1 & 765293 & $2.74-3.20$ & $10-12-19$ & 45.7 & $\begin{array}{l}\text { brown silty clay, trace of sand, } \\
\text { gravel }\end{array}$ \\
\hline 1 & & $3.20-3.66$ & & & \\
\hline 1 & & $3.66-4.12$ & & & \\
\hline 1 & & $4.12-4.57$ & & & \\
\hline 1 & $76 S 294$ & $4.57-5.03$ & $22-34-20$ & 15.2 & $\begin{array}{l}\text { brown silty clay to } 4.70 \mathrm{~m} ; 10.2 \mathrm{~cm} \\
\text { gravel layer }\end{array}$ \\
\hline 1 & & $5.03-5.49$ & & & \\
\hline 1 & & $5.49-5.94$ & & & \\
\hline
\end{tabular}


Table 2.--Test-well construction data and lithologic description of cores at Plot M--Continued

\section{Test well 21--Continued}

\begin{tabular}{|c|c|c|c|c|c|}
\hline $\begin{array}{l}\text { Unit } \\
\text { No. }\end{array}$ & $\begin{array}{l}\text { Sample } \\
\text { No. }\end{array}$ & $\begin{array}{c}\text { Depth } \\
\text { interval } \\
\text { (meters) }\end{array}$ & $\begin{array}{l}\text { Blow } \\
\text { counts }\end{array}$ & $\begin{array}{l}\text { Recovery } \\
\quad(\mathrm{cm})\end{array}$ & Description \\
\hline 1 & $76 \mathrm{~S} 295$ & $5.94-6.40$ & $9-14-24$ & 45.7 & $\begin{array}{l}\text { brown silty clay, trace of sand, } \\
\text { gravel }\end{array}$ \\
\hline 1 & & $6.40-6.96$ & & & \\
\hline 1 & & $6.96-7.32$ & & & \\
\hline 1 & $76 S 296$ & $7.32-7.77$ & $12-27-28$ & 45.7 & $\begin{array}{l}\text { brown silty clay, trace of sand, } \\
\text { gravel }\end{array}$ \\
\hline 2 & & $7.77-8.23$ & & & \\
\hline 2 & & $8.23-8.69$ & & & \\
\hline 2 & & $8.69-9.14$ & & & \\
\hline 2 & $76 S 297$ & $9.14-9.60$ & $9-14-19$ & 45.7 & $\begin{array}{l}\text { gray clayey silt, trace of sand, } \\
\text { gravel }\end{array}$ \\
\hline 2 & & $9.60-10.06$ & & & \\
\hline 2 & & $10.06-10.52$ & & & \\
\hline 2 & $76 S 298$ & $10.52-10.97$ & $8-13-16$ & 45.7 & $\begin{array}{l}\text { gray clayey silt w/gravel, trace of } \\
\text { sand }\end{array}$ \\
\hline 2 & & $10.97-11.43$ & & & \\
\hline 2 & & $11.43-11.89$ & & & \\
\hline 2 & $76 \$ 299$ & $11.89-12.34$ & $10-11-14$ & 45.7 & $\begin{array}{l}\text { gray clayey silt w/gravel, trace of } \\
\text { sand }\end{array}$ \\
\hline 2 & & $12.34-13.11$ & & & \\
\hline 2 & & $13.11-13.56$ & & & \\
\hline
\end{tabular}


Table 2.--Test-well construction data and lithologic description of cores at Plot M--Continued

Test well 21--Continued

\begin{tabular}{|c|c|c|c|c|c|}
\hline $\begin{array}{l}\text { Unit } \\
\text { No. }\end{array}$ & $\begin{array}{l}\text { Sample } \\
\text { No. }\end{array}$ & $\begin{array}{c}\text { Depth } \\
\text { interval } \\
\text { (meters) }\end{array}$ & $\begin{array}{l}\text { Blow } \\
\text { counts }\end{array}$ & $\begin{array}{l}\text { Recovery } \\
\quad(\mathrm{cm})\end{array}$ & Description \\
\hline 2 & $76 S 300$ & $13.56-14.02$ & $17-20-22$ & 45.7 & $\begin{array}{l}\text { gray clayey silt w/gravel, trace of } \\
\text { sand, to } 15.50 \mathrm{~m} \text {; layer of dolomite } \\
\text { gravel at bottom of sample }\end{array}$ \\
\hline 2 & & $14.02-14.48$ & & & \\
\hline 2 & & $14.48-14.94$ & & & \\
\hline 2 & $76 S 301$ & $14.94-15.39$ & $14-50-30$ & 45.7 & $\begin{array}{l}\text { gray clayey silt; grading to dolomite } \\
\text { gravel (12.7 cm); grading to grayish } \\
\text { brown clayey silt }\end{array}$ \\
\hline 3 & & $15.39-15.84$ & & & \\
\hline 3 & & $15.84-16.30$ & & & \\
\hline 3 & & $16.30-16.76$ & & & \\
\hline 3 & 765302 & $16.76-17.22$ & $32-38-54$ & 30.5 & $\begin{array}{l}\text { gray clayey silt to } 16.80 \mathrm{~m} \text {; contact } \\
\mathrm{w} / 20 \mathrm{~cm} \text { of gravel }\end{array}$ \\
\hline 3 & & $17.22-17.68$ & & & \\
\hline 3 & & $17.68-18.14$ & & & \\
\hline 3 & $76 S 303$ & $18.14-18.59$ & $20-26-29$ & 30.5 & $\begin{array}{l}\text { gray clayey silt, trace of sand, } \\
\text { gravel }\end{array}$ \\
\hline 3 & & $18.59-19.05$ & & & \\
\hline 3 & & $19.05-19.51$ & & & \\
\hline 4 & 765304 & $19.51-19.96$ & $17-17-18$ & 30.5 & $\begin{array}{l}\text { gray clayey silt, trace of sand, } \\
\text { gravel, wet }\end{array}$ \\
\hline 4 & & $19.96-20.42$ & & & \\
\hline 4 & & $20.42-20.88$ & & & \\
\hline
\end{tabular}


Table 2.--Test-well construction data and lithologic description of cores at Plot M--Continued

Test well 21--Continued

\begin{tabular}{lccccc}
\hline $\begin{array}{l}\text { Unit } \\
\text { No. }\end{array}$ & $\begin{array}{c}\text { Sample } \\
\text { No. }\end{array}$ & $\begin{array}{c}\text { Depth } \\
\text { interval } \\
\text { (meters) }\end{array}$ & $\begin{array}{c}\text { Blow } \\
\text { counts }\end{array}$ & $\begin{array}{c}\text { Recovery } \\
(\mathrm{cm})\end{array}$ & Description \\
\hline 4 & $20.88-21.34$ & & \\
\hline 4 & $76 \$ 305$ & $21.34-21.79$ & $17-23-50$ & 30.5 & $\begin{array}{l}\text { gray clayey silt to } 21.60 \mathrm{~m} \text {; contact } \\
\text { wand }\end{array}$ \\
\hline 4 & $21.79-22.25$ & \\
\hline 4 & $22.25-22.71$ \\
\hline 4 & 765306 & $22.71-22.86$ & $30-30-29$ & 30.5 & $\begin{array}{l}\text { gray clayey silt, trace of sand, } \\
\text { gravel }\end{array}$ \\
\hline
\end{tabular}

EOB $22.86 \mathrm{~m}$ 
Table 2.--Test-well construction data and lithologic description of cores at Plot M--Continued

\section{Test well 22}

Completion date: April 18, 1977

Location: 10 meters northeast of the center of Plot $M$, borehole passes through burial ground

Site and well construction data: test boring sealed to surface after drilling

Altitude of land surface............. $213.0 \mathrm{~m}$

\begin{tabular}{|c|c|c|c|c|c|}
\hline $\begin{array}{l}\text { Unit } \\
\text { No. }\end{array}$ & $\begin{array}{l}\text { Sample } \\
\text { No. }\end{array}$ & $\begin{array}{c}\text { Depth } \\
\text { interval } \\
\text { (meters) }\end{array}$ & $\begin{array}{l}\text { Blow } \\
\text { counts }\end{array}$ & $\begin{array}{l}\text { Recovery } \\
\quad(\mathrm{cm})\end{array}$ & Description \\
\hline 1 & & $0-0.46$ & & & trench cover and concrete cap \\
\hline 1 & & $0.46-0.91$ & & & \\
\hline 1 & 7751 & $0.91-1.37$ & -- & -- & yellow-brown silty clay \\
\hline 1 & & $1.37-1.83$ & & & \\
\hline 1 & 7752 & $1.83-2.29$ & -- & -- & yellow-brown clayey silt \\
\hline 1 & 7753 & $2.29-2.74$ & -- & -- & $\begin{array}{l}\text { yellow-brown clayey silt } w / \text { pebbles, } \\
\text { mottled }\end{array}$ \\
\hline 1 & 7754 & $2.74-3.20$ & -- & -- & brown clayey silt \\
\hline 1 & 7755 & $3.20-3.66$ & -- & -- & brown clayey silt w/pebbles \\
\hline 1 & 7756 & $3.66-4.12$ & -- & -- & yellow-brown clayey silt \\
\hline 1 & 7757 & $4.12-4.57$ & -- & -- & brown clayey silt w/few pebbles \\
\hline 1 & $77 \mathrm{~s} 8$ & $4.57-5.03$ & -- & -- & brown clayey silt, mottled \\
\hline 1 & 7759 & $5.03-5.49$ & -- & -- & $\begin{array}{l}\text { brown clayey silt w/few pebbles, } \\
\text { mottled }\end{array}$ \\
\hline 1 & 77510 & $5.49-5.94$ & -- & -- & $\begin{array}{l}\text { brown clayey silt } w / \text { few pebbles, } \\
\text { mottled }\end{array}$ \\
\hline 2 & & $5.94-6.40$ & & & \\
\hline
\end{tabular}


Table 2.--Test-well construction data and lithologic description of cores at Plot $\mathrm{M}$--Continued

Test well 22--Continued

\begin{tabular}{|c|c|c|c|c|c|}
\hline $\begin{array}{l}\text { Unit } \\
\text { No. }\end{array}$ & $\begin{array}{l}\text { Sample } \\
\text { No. }\end{array}$ & $\begin{array}{c}\text { Depth } \\
\text { interval } \\
\text { (meters) }\end{array}$ & $\begin{array}{l}\text { Blow } \\
\text { counts }\end{array}$ & $\begin{array}{l}\text { Recovery } \\
\quad(\mathrm{cm})\end{array}$ & Description \\
\hline 2 & & $6.40-6.96$ & & & \\
\hline 2 & & $6.96-7.32$ & & & \\
\hline 2 & $77 \mathrm{~S} 11$ & $7.32-7.77$ & -- & - & $\begin{array}{l}\text { brown clayey silt } w / \text { few pebbles, } \\
\text { mottled }\end{array}$ \\
\hline 2 & & $7.77-8.23$ & & & \\
\hline 2 & & $8.23-8.69$ & & & \\
\hline 2 & & $8.69-9.14$ & & & \\
\hline 2 & $77 \mathrm{~S} 12$ & $9.14-9.60$ & -- & -- & brown clayey silt \\
\hline 2 & & $9.60-10.06$ & & & \\
\hline 2 & & $10.06-10.52$ & & & \\
\hline 2 & $77 \mathrm{SI} 3$ & $10.52-10.97$ & -- & -- & gray-brown clayey silt w/few pebbles \\
\hline 2 & & $10.97-11.43$ & & & \\
\hline 2 & & $11.43-11.89$ & & & \\
\hline 2 & $77 S 14$ & $11.89-12.34$ & -- & -- & $\begin{array}{l}\text { gray-brown clayey silt grading to } \\
\text { gray clayey silt w/pebbles, mostly } \\
\text { dolomite }\end{array}$ \\
\hline 2 & & $12.34-13.11$ & & & \\
\hline 2 & & $13.11-13.56$ & & & \\
\hline 2 & $77 \mathrm{~S} 15$ & $13.56-14.02$ & -- & -- & $\begin{array}{l}\text { gray clayey silt w/pebbles, mostly } \\
\text { dolomite }\end{array}$ \\
\hline 2 & & $14.02-14.48$ & & & \\
\hline 2 & & $14.48-14.94$ & & & \\
\hline
\end{tabular}


Table 2.--Test-well construction data and lithologic description of cores at Plot M--Continued

\section{Test well 22--Continued}

\begin{tabular}{|c|c|c|c|c|c|}
\hline $\begin{array}{l}\text { Unit } \\
\text { No. }\end{array}$ & $\begin{array}{l}\text { Sample } \\
\text { No. }\end{array}$ & $\begin{array}{c}\text { Depth } \\
\text { interval } \\
\text { (meters) }\end{array}$ & $\begin{array}{l}\text { Blow } \\
\text { counts }\end{array}$ & $\begin{array}{l}\text { Recovery } \\
\quad(\mathrm{cm})\end{array}$ & Description \\
\hline 3 & $77 \mathrm{~S} 16$ & $14.94-15.39$ & -- & -- & gray clayey silt $w /$ few pebbles \\
\hline 3 & & $15.39-15.85$ & & & \\
\hline 3 & & $15.85-16.31$ & & & \\
\hline 3 & $77 S 17$ & $16.31-16.76$ & -- & - & $\begin{array}{l}\text { gray clayey silt w/few limestone } \\
\text { pebbles }\end{array}$ \\
\hline 3 & & $16.76-17.22$ & & & \\
\hline 3 & & $17.22-17.68$ & & & \\
\hline 3 & & $17.68-18.14$ & & & \\
\hline 3 & 77518 & $18.14-18.59$ & -- & -- & $\begin{array}{l}\text { gray clayey silt; } 1.9 \mathrm{~cm} \text { sand layer } \\
\text { at } 18.59 \mathrm{~m}\end{array}$ \\
\hline 3 & & $18.59-19.05$ & & & \\
\hline 3 & & $19.05-19.51$ & & & \\
\hline 3 & $77 S 19$ & $19.51-19.96$ & -- & -- & $\begin{array}{l}\text { gray clayey silt w/pebbles, mostly } \\
\text { dolomite }\end{array}$ \\
\hline 3 & & $19.96-20.42$ & & & \\
\hline 3 & & $20.42-20.88$ & & & \\
\hline 3 & & $20.88-21.33$ & & & \\
\hline 3 & 77520 & $21.33-21.79$ & -- & -- & gray silt $w /$ few pebbles \\
\hline 4 & & $21.79-22.25$ & & & \\
\hline 4 & & $22.25-22.71$ & & & \\
\hline 4 & $77 \mathrm{~S} 21$ & $22.71-23.16$ & -- & -- & gray silt $w /$ few pebbles, stones \\
\hline
\end{tabular}


Table 2.--Test-well construction data and lithologic description of cores at Plot $\mathrm{M}$--Continued

Test well 22--Continued

\begin{tabular}{lccccc}
\hline $\begin{array}{l}\text { Unit } \\
\text { No. }\end{array}$ & $\begin{array}{c}\text { Sample } \\
\text { No. }\end{array}$ & $\begin{array}{c}\text { Depth } \\
\text { interval } \\
\text { (meters) }\end{array}$ & $\begin{array}{c}\text { Blow } \\
\text { counts }\end{array}$ & $\begin{array}{c}\text { Recovery } \\
(\mathrm{cm})\end{array}$ & Description \\
\hline 4 & $23.16-23.62$ & & \\
\hline 4 & $23.62-24.08$ & -0 & gray silt \\
\hline 4 & 77522 & $24.08-24.53$ & - & \\
\hline 5
\end{tabular}


Table 2.--Test-well construction data and lithologic description of cores at Plot M--Continued

Test well 23

\section{Completion date:}

Location: 5 meters east of center of Plot M, borehole passes through burial ground Site and well construction data: test boring sealed to surface after drilling

Altitude of land surface . . . . . . . . . . . $213.40 \mathrm{~m}$

\begin{tabular}{|c|c|c|c|c|c|}
\hline $\begin{array}{l}\text { Unit } \\
\text { No. }\end{array}$ & $\begin{array}{l}\text { Sample } \\
\text { No. }\end{array}$ & $\begin{array}{c}\text { Depth } \\
\text { interval } \\
\text { (meters) }\end{array}$ & $\begin{array}{l}\text { Blow } \\
\text { counts }\end{array}$ & $\begin{array}{l}\text { Recovery } \\
\quad(\mathrm{cm})\end{array}$ & Description \\
\hline 1 & & $0-0.46$ & & & trench cover and concrete cap \\
\hline 1 & & $0.46-0.91$ & & & \\
\hline 1 & & $0.91-1.37$ & -- & -- & $\begin{array}{l}\text { gray-black silty clay grading to } \\
\text { yellow-brown silty clay }\end{array}$ \\
\hline 1 & 77524 & $1.37-1.83$ & -- & -- & $\begin{array}{l}\text { yellow-gray silty clay grading to } \\
\text { yellowish brown silty clay }\end{array}$ \\
\hline 1 & $77 \mathrm{~S} 25$ & $1.83-2.29$ & -- & -- & $\begin{array}{l}\text { yellow-gray silty clay; } 1.3 \mathrm{~cm} \text { piece } \\
\text { of graphite }\end{array}$ \\
\hline 1 & $77 \mathrm{~S} 26$ & $2.29-2.74$ & -- & -- & yellow-brown silty clay, mottled \\
\hline 1 & $77 \mathrm{~S} 27$ & $2.74-3.20$ & -- & -- & brown silty clay w/pebbles, mottled \\
\hline 1 & 77528 & $3.20-3.66$ & -- & -- & brown silty clay, mottled \\
\hline 1 & $77 \mathrm{~S} 29$ & $3.66-4.12$ & -- & -- & $\begin{array}{l}\text { brown silty clay, mottled; } 1.32 \mathrm{~m} \\
\text { sand parting at } 4.00 \mathrm{~m}\end{array}$ \\
\hline 1 & 77530 & $4.12-4.57$ & -- & -- & $\begin{array}{l}\text { gray clayey silt w/pebbles, mostly } \\
\text { dolomite }\end{array}$ \\
\hline 1 & $77 \mathrm{~S} 31$ & $4.57-5.03$ & -- & -- & gray clayey silt \\
\hline 1 & $77 \mathrm{~S} 32$ & $5.03-5.49$ & -- & -- & $\begin{array}{l}\text { gray clayey silt; grading to yellow- } \\
\text { gray clayey silt w/pebbles }\end{array}$ \\
\hline 1 & $77 \mathrm{~S} 33$ & $5.49-5.94$ & -- & -- & $\begin{array}{l}\text { gray clayey silt w/pebbles, stones, } \\
\text { mostly dolomite }\end{array}$ \\
\hline
\end{tabular}


Table 2.--Test-well construction data and lithologic description of cores at Plot M--Continued

Test well 23--Continued

\begin{tabular}{|c|c|c|c|c|c|}
\hline $\begin{array}{l}\text { Unit } \\
\text { No. }\end{array}$ & $\begin{array}{c}\text { Sample } \\
\text { No. }\end{array}$ & $\begin{array}{c}\text { Depth } \\
\text { interval } \\
\text { (meters) }\end{array}$ & $\begin{array}{l}\text { Blow } \\
\text { counts }\end{array}$ & $\begin{array}{l}\text { Recovery } \\
(\mathrm{cm})\end{array}$ & Description \\
\hline 1 & & $5.94-6.40$ & & & \\
\hline 1 & & $6.40-6.96$ & & & \\
\hline 1 & & $6.96-7.32$ & & & \\
\hline 2 & 77534 & $7.32-7.77$ & -- & -- & gray clayey silt \\
\hline 2 & & $7.77-8.23$ & & & \\
\hline 2 & & $8.23-8.69$ & & & \\
\hline 2 & & $8.69-9.14$ & & & \\
\hline 2 & $77 \mathrm{~S} 35$ & $9.14-9.60$ & -- & -- & gray clayey silt w/pebbles \\
\hline 2 & & $9.60-10.06$ & & & \\
\hline 2 & & $10.06-10.52$ & & & \\
\hline 2 & 77536 & $10.52-10.97$ & -- & -- & gray clayey silt $\mathrm{w} /$ numerous pebbles \\
\hline 2 & & $10.97-11.43$ & & & \\
\hline 2 & & $11.43-11.89$ & & & \\
\hline 2 & 77537 & $11.89-12.34$ & -- & -- & brown silty clay \\
\hline 2 & & $12.34-13.11$ & & & \\
\hline 2 & & $13.11-13.56$ & & & \\
\hline 2 & 77538 & $13.56-14.02$ & -- & -- & gray-brown silty clay w/pebbles \\
\hline 3 & & $14.02-14.48$ & & & \\
\hline 3 & & $14.48-14.94$ & & & \\
\hline 3 & 77539 & $14.94-15.39$ & -- & -- & brown silt w/pebbles, sandy \\
\hline
\end{tabular}


Table 2.--Test-well construction data and lithologic description of cores at Plot M--Continued

\section{Test well 23--Continued}

\begin{tabular}{|c|c|c|c|c|c|}
\hline $\begin{array}{l}\text { Unit } \\
\text { No. }\end{array}$ & $\begin{array}{l}\text { Sample } \\
\text { No. }\end{array}$ & $\begin{array}{c}\text { Depth } \\
\text { interval } \\
\text { (meters) }\end{array}$ & $\begin{array}{l}\text { Blow } \\
\text { counts }\end{array}$ & $\begin{array}{l}\text { Recovery } \\
\quad(\mathrm{cm})\end{array}$ & Description \\
\hline 3 & & $15.39-15.85$ & & & \\
\hline 3 & & $15.85-16.31$ & & & \\
\hline 3 & & $16.31-16.76$ & & & \\
\hline 3 & 77540 & $16.76-17.22$ & -- & - & $\begin{array}{l}\text { gray clayey silt to } 17.10 \mathrm{~m} \text {; contact } \\
\text { w/gray silt }\end{array}$ \\
\hline 3 & & $17.22-17.68$ & & & \\
\hline 3 & & $17.68-18.14$ & & & \\
\hline 3 & 77541 & $18.14-18.59$ & -- & -- & gray clayey silt, grading to gray silt \\
\hline 3 & & $18.59-19.05$ & & & \\
\hline 3 & & $19.05-19.51$ & & & \\
\hline 3 & $77 \mathrm{~S} 42$ & $19.51-19.96$ & -- & -- & gray clayey silt w/stones \\
\hline 3 & & $19.96-20.42$ & & & \\
\hline 3 & & $20.42-20.88$ & & & . \\
\hline 3 & & $20.88-21.33$ & & & \\
\hline 3 & 77543 & $21.33-21.79$ & -- & -- & brown clayey silt $w /$ few pebbles \\
\hline 4 & & $21.79-22.25$ & & & \\
\hline 4 & & $22.25-22.71$ & & & \\
\hline 4 & 77544 & $22.71-23.16$ & -- & -- & gray clayey silt $w /$ few pebbles \\
\hline 4 & & $23.16-23.62$ & & & \\
\hline 4 & & $23.62-24.08$ & & & \\
\hline
\end{tabular}


Table 2.--Test-well construction data and lithologic description of cores at Plot M--Continued

Test well 23--Continued

\begin{tabular}{|c|c|c|c|c|c|}
\hline $\begin{array}{l}\text { Unit } \\
\text { No. }\end{array}$ & $\begin{array}{c}\text { Sample } \\
\text { No. }\end{array}$ & $\begin{array}{c}\text { Depth } \\
\text { interval } \\
\text { (meters) }\end{array}$ & $\begin{array}{l}\text { Blow } \\
\text { counts }\end{array}$ & $\begin{array}{l}\text { Recovery } \\
\text { (cm) }\end{array}$ & Description \\
\hline 4 & $77 S 45$ & $24.08-24.53$ & -- & -- & $\begin{array}{l}\text { gray clayey silt w/pebbles, stones, } \\
\text { mostly dolomite }\end{array}$ \\
\hline 4 & & $24.53-24.99$ & & & \\
\hline 4 & & $24.99-25.45$ & & & \\
\hline 4 & 77546 & $25.45-25.91$ & -- & -- & gray silt \\
\hline 4 & & $25.91-26.37$ & & & \\
\hline 4 & & $26.37-26.82$ & & & \\
\hline 4 & & $26.82-27.28$ & & & \\
\hline 4 & 77547 & $27.28-27.74$ & -- & -- & gray silt \\
\hline 4 & & $27.74-28.19$ & & & \\
\hline 4 & & $28.19-28.65$ & & & \\
\hline 4 & 77548 & $28.65-29.11$ & -- & -- & gray silt \\
\hline 4 & & $29.11-29.57$ & & & \\
\hline 4 & & $29.57-30.02$ & & & \\
\hline 4 & & $30.02-30.48$ & & & \\
\hline 4 & 77549 & $30.48-30.93$ & -- & -- & gray silt \\
\hline
\end{tabular}


Table 2.--Test-well construction data and lithologic description of cores at Plot M--Continued

\section{Test well 24}

Completion date: May 3, 1978

Location: Next to test well 2, 26 meters north of the center of Plot $M$

Site and well construction data: 2 test wells; a $22.86 \mathrm{~m}$ test well west of test well 2 and a $38.10 \mathrm{~m}$ test well east of test well 2

Altitude of land surface: $22.86 \mathrm{~m}$ test well. . . . . . . $211.07 \mathrm{~m}$

$38.10 \mathrm{~m}$ test well. ....... $211.01 \mathrm{~m}$

Altitude of measuring point (top of casing): $22.86 \mathrm{~m}$ test well. . $211.16 \mathrm{~m}$

$38.10 \mathrm{~m}$ test well. $211.02 \mathrm{~m}$

Depth of bottom of piezometer from land surface: $22.86 \mathrm{~m}$ test well. $.22 .77 \mathrm{~m}$

$38.10 \mathrm{~m}$ test well. $38.09 \mathrm{~m}$

Type of piezometer: $1.52 \mathrm{~m}$ perforated PVC pipe, $6.35 \mathrm{~cm}$ diameter

\begin{tabular}{|c|c|c|c|c|c|}
\hline $\begin{array}{l}\text { Unit } \\
\text { No. }\end{array}$ & $\begin{array}{l}\text { Sample } \\
\text { No. }\end{array}$ & $\begin{array}{c}\text { Depth } \\
\text { interval } \\
\text { (meters) }\end{array}$ & $\begin{array}{l}\text { Blow } \\
\text { counts }\end{array}$ & $\begin{array}{l}\text { Recovery } \\
\text { (cm) }\end{array}$ & Description \\
\hline 1 & & $0-0.46$ & & & \\
\hline 1 & & $0.46-0.91$ & & & \\
\hline 1 & & $0.91-1.37$ & & & \\
\hline 1 & 7851 & $1.37-1.83$ & $9-14-17$ & 45.7 & brown silty clay $\mathrm{w} /$ weathered pebbles \\
\hline 1 & & $1.83-2.29$ & & & \\
\hline 1 & & $2.29-2.74$ & & & \\
\hline 1 & 7852 & $2.74-3.20$ & $10-11-12$ & 61.0 & brown silty clay $w /$ pebbles \\
\hline 1 & & $3.20-3.66$ & & & \\
\hline 1 & & $3.66-4.12$ & & & \\
\hline 1 & & $4.12-4.57$ & & & \\
\hline 1 & 7853 & $4.57-5.03$ & $7-10-11$ & 53.3 & $\begin{array}{l}\text { brown silty clay w/pebbles, stones, } \\
\text { sandy }\end{array}$ \\
\hline 1 & & $5.03-5.49$ & & & \\
\hline
\end{tabular}


Table 2.--Test-well construction data and lithologic description of cores at Plot M--Continued

Test well 24--Continued

\begin{tabular}{|c|c|c|c|c|c|}
\hline $\begin{array}{l}\text { Unit } \\
\text { No. }\end{array}$ & $\begin{array}{l}\text { Sample } \\
\text { No. }\end{array}$ & $\begin{array}{c}\text { Depth } \\
\text { interval } \\
\text { (meters) }\end{array}$ & $\begin{array}{l}\text { Blow } \\
\text { counts }\end{array}$ & $\begin{array}{l}\text { Recovery } \\
\quad(\mathrm{cm})\end{array}$ & Description \\
\hline 1 & & $5.49-5.94$ & & & \\
\hline 1 & 7854 & $5.94-6.40$ & $15-17-24$ & 61.0 & $\begin{array}{l}\text { brown silty clay w/pebbles to } 6.55 \mathrm{~m} \text {; } \\
\text { contact w/gray silty clay to } 7.62 \mathrm{~m}\end{array}$ \\
\hline 1 & & $6.40-6.96$ & & & \\
\hline 2 & & $6.96-7.32$ & & & \\
\hline 2 & & $7.32-7.77$ & & & \\
\hline 2 & 7855 & $7.77-8.23$ & $21-18-23$ & 61.0 & $\begin{array}{l}\text { brown silty clay w/pebbles to } 7.77 \mathrm{~m} \text {; } \\
\text { gray-brown silty clay w/pebbles, } \\
\text { stones, gritty }\end{array}$ \\
\hline 2 & & $8.23-8.69$ & & & \\
\hline 2 & & $8.69-9.14$ & & & \\
\hline 2 & 7856 & $9.14-9.60$ & $15-18-22$ & 45.7 & gray-brown silty clay \\
\hline 2 & & $9.60-10.06$ & & & \\
\hline 2 & & $10.06-10.52$ & & & \\
\hline 2 & 7857 & $10.52-10.97$ & $8-11-25$ & 45.7 & $\begin{array}{l}\text { gray-brown clayey silt; grading to } \\
\text { brown, red-gray clayey silt }\end{array}$ \\
\hline 2 & & $10.97-11.43$ & & & \\
\hline 2 & & $11.43-11.89$ & & & \\
\hline 2 & & $11.89-12.34$ & & & \\
\hline 3 & 7858 & $12.34-13.11$ & $8-13-18$ & 45.7 & $\begin{array}{l}\text { sandy gravelly, clayey silt at } 12.39 \mathrm{~m} \text {; } \\
\text { grading to grayish brown clayey silt }\end{array}$ \\
\hline 3 & & $13.11-13.56$ & & & \\
\hline
\end{tabular}


Table 2.--Test-well construction data and lithologic description of cores at Plot M--Continued

Test well 24--Continued

\begin{tabular}{|c|c|c|c|c|c|}
\hline $\begin{array}{l}\text { Unit } \\
\text { No. }\end{array}$ & $\begin{array}{l}\text { Sample } \\
\text { No. }\end{array}$ & $\begin{array}{c}\text { Depth } \\
\text { interval } \\
\text { (meters) }\end{array}$ & $\begin{array}{l}\text { Blow } \\
\text { counts }\end{array}$ & $\begin{array}{l}\text { Recovery } \\
\quad(\mathrm{cm})\end{array}$ & Description \\
\hline 3 & 7859 & $13.56-14.02$ & $8-10-14$ & 45.7 & gray-brown clayey silt \\
\hline 3 & & $14.02-14.48$ & & & \\
\hline 3 & & $14.48-14.94$ & & & \\
\hline 3 & & $14.94-15.39$ & & & \\
\hline 3 & $78 S 10$ & $15.39-15.85$ & $10-19-27$ & 45.7 & $\begin{array}{l}\text { gray clayey silt w/pebbles, gritty, } \\
\text { mostly dolomite grains }\end{array}$ \\
\hline 3 & & $15.85-16.31$ & & & \\
\hline 3 & & $16.31-16.76$ & & & \\
\hline 3 & 78511 & $16.76-17.22$ & & 7.6 & $\begin{array}{l}\text { brown-gray clayey silt w/pebbles, } \\
\text { stones, moist }\end{array}$ \\
\hline 3 & & $17.22-17.68$ & & & \\
\hline 3 & & $17.68-18.14$ & & & \\
\hline 3 & $78 S 12$ & $18.14-18.59$ & $20-21-22$ & 45.7 & $\begin{array}{l}\text { brown-gray clayey silt, moist, } 17.8 \\
\mathrm{~cm} \text { sandy, clayey silt at } 18.44 \mathrm{~m}\end{array}$ \\
\hline 3 & & $18.59-19.05$ & & & \\
\hline 3 & & $19.05-19.51$ & & & \\
\hline 3 & $78 S 13$ & $19.51-19.96$ & $11-17-16$ & 30.5 & $\begin{array}{l}\text { brown-gray clayey silt } w / \text { few pebbles, } \\
\text { stones }\end{array}$ \\
\hline 3 & & $19.96-20.42$ & & & \\
\hline 3 & & $20.42-20.88$ & & & \\
\hline 4 & & $20.88-21.33$ & & & \\
\hline 4 & 78514 & $21.33-21.79$ & $15-28-32$ & 45.7 & $\begin{array}{l}\text { gray clayey silt, no pebbles (clean), } \\
\text { dry }\end{array}$ \\
\hline
\end{tabular}


Table 2.--Test-well construction data and lithologic description of cores at Plot M--Continued

Test well 24--Continued

\begin{tabular}{|c|c|c|c|c|c|}
\hline $\begin{array}{l}\text { Unit } \\
\text { No. }\end{array}$ & $\begin{array}{c}\text { Sample } \\
\text { No. }\end{array}$ & $\begin{array}{c}\text { Depth } \\
\text { interval } \\
\text { (meters) }\end{array}$ & $\begin{array}{l}\text { Blow } \\
\text { counts }\end{array}$ & $\begin{array}{l}\text { Recovery } \\
(\mathrm{cm})\end{array}$ & Description \\
\hline 4 & & $21.79-22.25$ & & & \\
\hline 4 & & $22.25-22.71$ & & & \\
\hline 4 & 78515 & $22.71-23.16$ & $18-29-27$ & 35.6 & gray clayey silt $w /$ few pebbles \\
\hline 4 & & $23.16-23.62$ & & & \\
\hline 4 & & $23.62-24.08$ & & & \\
\hline 4 & $78 S 16$ & $24.08-24.53$ & $22-28-39$ & 15.2 & gray clayey silt, clean, dry \\
\hline 4 & & $24.53-24.99$ & & & \\
\hline 4 & & $24.99-25.45$ & & & \\
\hline 4 & & $25.45-25.91$ & & & \\
\hline 4 & $78 S 17$ & $25.91-26.37$ & $22-43-45$ & 17.8 & gray clayey silt, clean, dry \\
\hline 4 & & $26.37-26.82$ & & & \\
\hline 5 & & $26.82-27.28$ & & & \\
\hline 5 & 78518 & $27.28-27.74$ & $35-90$ & 17.8 & gray clayey silt \\
\hline 5 & & $27.74-28.19$ & & & \\
\hline 5 & & $28.19-28.65$ & & & \\
\hline 5 & 78519 & $28.65-29.11$ & $29-55-60$ & 26.7 & gray clayey silt \\
\hline 5 & & $29.11-29.57$ & & & \\
\hline 5 & & $29.57-30.02$ & & & \\
\hline 5 & & $30.02-30.48$ & & & \\
\hline 5 & $78 \$ 20$ & $30.48-30.94$ & $31-75$ & 30.5 & gray clayey silt \\
\hline
\end{tabular}


Table 2.--Test-well construction data and lithologic description of cores at Plot $\mathrm{M}-$-Continued

Test well 24--Continued

\begin{tabular}{|c|c|c|c|c|c|}
\hline $\begin{array}{l}\text { Unit } \\
\text { No. }\end{array}$ & $\begin{array}{l}\text { Sample } \\
\text { No. }\end{array}$ & $\begin{array}{c}\text { Depth } \\
\text { interval } \\
\text { (meters) }\end{array}$ & $\begin{array}{l}\text { Blow } \\
\text { counts }\end{array}$ & $\begin{array}{l}\text { Recovery } \\
\quad(\mathrm{cm})\end{array}$ & Description \\
\hline 5 & & $30.94-31.39$ & & & \\
\hline 5 & & $31.39-31.85$ & & & \\
\hline 5 & 78521 & $31.85-32.31$ & $20-26-27$ & 30.5 & gray clayey silt \\
\hline 5 & & $32.31-33.22$ & & & \\
\hline 5 & & $33.22-33.68$ & & & \\
\hline 6 & 78522 & $33.68-34.14$ & $12-55-48$ & 27.9 & $\begin{array}{l}\text { brown silt, pebbles, stones, sandy; } \\
\text { grading to gray clayey silt }\end{array}$ \\
\hline 6 & & $34.14-34.60$ & & & \\
\hline 6 & & $34.60-3.5 .05$ & & & \\
\hline 6 & 78523 & $35.05-35.51$ & $9-13-15$ & 17.8 & brown-gray silt $w /$ large stones \\
\hline 6 & & $35.51-35.97$ & & & \\
\hline 6 & & $35.97-36.42$ & & & \\
\hline 6 & 78524 & $36.42-36.88$ & $35-35-46$ & 45.7 & gray silt $\mathrm{w} /$ numerous stones \\
\hline 6 & & $36.88-37.34$ & & & \\
\hline 6 & & $37.34-37.80$ & & & \\
\hline 6 & & $37.80-38.25$ & & & \\
\hline 6 & 78525 & $38.25-38.71$ & $48-48-58$ & 17.8 & gray silt $\mathrm{w} /$ numerous pebbles, stones \\
\hline & & EOB $38.71 \mathrm{~m}$ & & & \\
\hline
\end{tabular}


Table 2.--Test-well construction data and lithologic description of cores at Plot M--Continued

\section{Test well 25}

Completion date: October 5, 1979

Location: 33 meters west of test well 26, 55 meters south of test well 27

Site and well construction data:

Altitude of land surface............. $216.10 \mathrm{~m}$

Altitude of measuring point (top of casing). . . . $216.20 \mathrm{~m}$

Depth of bottom of piezometer from land surface. . . $18.34 \mathrm{~m}$

Type of piezometer: $1.22 \mathrm{~m}$ slotted PVC pipe, $5.08 \mathrm{~cm}$ diameter

\begin{tabular}{|c|c|c|c|c|c|}
\hline $\begin{array}{l}\text { Unit } \\
\text { No. }\end{array}$ & $\begin{array}{c}\text { Sample } \\
\text { No. }\end{array}$ & $\begin{array}{c}\text { Depth } \\
\text { interval } \\
\text { (meters) }\end{array}$ & $\begin{array}{l}\text { Blow } \\
\text { counts }\end{array}$ & $\begin{array}{l}\text { Recovery } \\
\text { (cm). }\end{array}$ & Description \\
\hline 1 & & $0-0.46$ & & & \\
\hline 1 & & $0.46-0.91$ & & & \\
\hline 1 & & $0.91-1.37$ & & & \\
\hline 1 & & $1.37-1.83$ & & & \\
\hline 1 & & $1.83-2.29$ & & & \\
\hline 1 & & $2.29-2.74$ & & & \\
\hline 1 & & $2.74-3.20$ & & & \\
\hline 1 & & $3.20-3.66$ & & & \\
\hline 1 & & $3.66-4.12$ & & & \\
\hline 1 & & $4.12-4.57$ & & & \\
\hline 1 & & $4.57-5.03$ & & & \\
\hline 1 & & $5.03-5.49$ & & & \\
\hline 1 & & $5.49-5.94$ & & & \\
\hline 1 & 795212 & $5.94-6.40$ & $16-19-50 / 5$ & 50.5 & $\begin{array}{l}\text { yellow-brown silty clay w/pebbles } \\
\text { (large variety), gritty, very hard, } \\
\text { dense, dry, sand partings coated w/ } \\
\text { plant fragments; } 45^{\circ} \text { oxidized fracture }\end{array}$ \\
\hline
\end{tabular}


Table 2.--Test-well construction data and lithologic description of cores at Plot M--Continued

Test well 25--Continued

\begin{tabular}{|c|c|c|c|c|c|}
\hline $\begin{array}{l}\text { Unit } \\
\text { No. }\end{array}$ & $\begin{array}{l}\text { Sample } \\
\text { No. }\end{array}$ & $\begin{array}{c}\text { Depth } \\
\text { interval } \\
\text { (meters) }\end{array}$ & $\begin{array}{l}\text { Blow } \\
\text { counts }\end{array}$ & $\begin{array}{l}\text { Recovery } \\
(\mathrm{cm})\end{array}$ & Description \\
\hline 1 & & $6.40-6.86$ & & & \\
\hline 1 & & $6.86-7.32$ & & & \\
\hline 1 & 795213 & $7.32-7.77$ & $17-22-25$ & 35.6 & $\begin{array}{l}\text { yellow-brown silty clay w/pebbles } \\
\text { (large variety); pyrite; shell } \\
\text { fragments }\end{array}$ \\
\hline 1 & & $7.77-8.23$ & & & \\
\hline 1 & & $8.23-8.69$ & & & \\
\hline 1 & 795214 & $8.69-9.14$ & $40-36-34$ & 35.6 & $\begin{array}{l}\text { yellow-brown silty clay w/pebbles, } \\
\text { large stones, mottled, brittle, dry; } \\
\text { orange-brown oxidized partings }\end{array}$ \\
\hline 1 & & $9.14-9.60$ & & & \\
\hline 1 & & $9.60-10.06$ & & & \\
\hline 1 & & $10.06-10.52$ & & & \\
\hline 1 & 795215 & $10.52-10.97$ & $34-28-26$ & 20.3 & $\begin{array}{l}\text { yellow-brown clayey silt w/pebbles, } \\
\text { stones, rocks, dry, flaky, very hard, } \\
5.1 \mathrm{~cm} \text { orange-brown oxidized zone }\end{array}$ \\
\hline 1 & & $10.97-11.43$ & & & \\
\hline 1 & & $11.43-11.89$ & & & \\
\hline 1 & 795216 & $11.89-12.34$ & $14-19-20$ & 20.3 & $\begin{array}{l}\text { yellow-brown clayey silt w/limestone } \\
\text { pebbles, stones, hard }\end{array}$ \\
\hline 1 & & $12.34-12.80$ & & & \\
\hline 1 & & $12.80-13.26$ & & & \\
\hline 1 & & $13.26-13.72$ & & & \\
\hline
\end{tabular}


Table 2.--Test-well construction data and lithologic description of cores at Plot M--Continued

Test well 25--Continued

\begin{tabular}{|c|c|c|c|c|c|}
\hline $\begin{array}{l}\text { Unit } \\
\text { No. }\end{array}$ & $\begin{array}{c}\text { Sample } \\
\text { No. }\end{array}$ & $\begin{array}{c}\text { Depth } \\
\text { interval } \\
\text { (meters) }\end{array}$ & $\begin{array}{l}\text { Blow } \\
\text { counts }\end{array}$ & $\begin{array}{l}\text { Recovery } \\
\quad(\mathrm{cm})\end{array}$ & Description \\
\hline 1 & 795217 & $13.72-14.17$ & $15-17-19$ & 45.7 & $\begin{array}{l}\text { yellow-brown clayey silt to } 14.17 \mathrm{~m} \text {; } \\
\text { contact w/gray clayey silt w/pebbles }\end{array}$ \\
\hline 2 & & $14.17-14.63$ & & & \\
\hline 2 & & $14.63-15.09$ & & & \\
\hline 2 & 795218 & $15.09-15.55$ & $14-17-17$ & 50.5 & $\begin{array}{l}\text { dark gray clayey silt w/few pebbles; } \\
\text { sand parting at } 15.39 \mathrm{~m} \text {; two graded } \\
\text { silt units in last } 15 \mathrm{~cm} \text { are moist, } \\
\text { soft, dense }\end{array}$ \\
\hline 2 & & $15.55-16.00$ & & & \\
\hline 2 & & $16.00-16.46$ & & & \\
\hline 2 & 795219 & $16.46-16.92$ & $11-14-17$ & 35.6 & $\begin{array}{l}\text { gray clayey silt } w / \text { few pebbles, soft, } \\
\text { mostly dolomite grains }\end{array}$ \\
\hline 2 & & $16.92-17.37$ & & & \\
\hline 2 & 795220 & $17.37-17.83$ & $14-18-21$ & 40.6 & $\begin{array}{l}\text { gray clayey silt w/few pebbles, } \\
\text { mostly dolomite grains }\end{array}$ \\
\hline
\end{tabular}

EOB $19.81 \mathrm{~m}$ 
Table 2.--Test-well construction data and lithologic description of cores at Plot M--Continued

Test well 26

Completion date: October 9, 1979

Location: 10 meters northeast of the northeast corner of Plot M Site and well construction data:

Altitude of land surface . . . . . . . . . $210.77 \mathrm{~m}$

Altitude of measuring point (top of casing). . . . $210.99 \mathrm{~m}$

Depth of bottom of piezometer from land surface. . . $18.22 \mathrm{~m}$

Type of piezometer: $1.22 \mathrm{~m}$ slotted PVC pipe, $5.08 \mathrm{~cm}$ diameter

\begin{tabular}{cccccl}
\hline $\begin{array}{c}\text { Unit } \\
\text { No. }\end{array}$ & $\begin{array}{c}\text { Sample } \\
\text { No. }\end{array}$ & $\begin{array}{c}\text { Depth } \\
\text { interval } \\
\text { (meters) }\end{array}$ & $\begin{array}{c}\text { Blow } \\
\text { counts }\end{array}$ & $\begin{array}{c}\text { Recovery } \\
(\mathrm{cm})\end{array}$ & Description \\
\hline 1 & $79 \mathrm{~S} 221$ & $0-0.46$ & $8-12-15$ & 15.2 & brown stoney clay soil \\
\hline 1 & 795222 & $0.46-0.91$ & $11-10-18$ & 15.2 & $\begin{array}{l}\text { brown clayey soil, root tubes, plant } \\
\text { fragments }\end{array}$ \\
\hline 1 & 795223 & $0.91-1.37$ & $20-20-24$ & 25.4 & $\begin{array}{l}\text { brown clayey soil w/stones, plant } \\
\text { fragments; } 2.5 \mathrm{~cm} \text { dry yellow sand } \\
\text { layer at } 1.16 \mathrm{~m}\end{array}$ \\
\hline
\end{tabular}

\begin{tabular}{llllll}
1 & 795224 & $1.37-1.83$ & $13-18-21$ & 25.4 & $\begin{array}{l}\text { yellow-brown silty clay w/pebbles, } \\
\text { mottled, hard, dry, flaky, brittle }\end{array}$ \\
\hline 1 & 795225 & $1.83-2.29$ & $23-25-29$ & 25.4 & $\begin{array}{l}\text { yellow-brown silty clay w/pebbles } \\
\text { (large variety), flaky, brittle; } \\
\text { plant fragments, Fe oxidation, pyrite; } \\
45^{\circ} \text { fracture at } 1.83 \mathrm{~m}\end{array}$ \\
\hline
\end{tabular}

$1795226 \quad 2.29-2.74 \quad 14-20-25 \quad 61.0$ yellow-brown silty clay w/pebbles
(large variety); vertical fracture $(25.4 \mathrm{~cm})$ oxidized $\mathrm{w} / \mathrm{plant}$ debris

\begin{tabular}{|c|c|c|c|c|c|}
\hline 1 & 795227 & $2.74-3.20$ & $14-20-26$ & 45.7 & brown silty clay w/pebbles, hard, dry \\
\hline 1 & 795228 & $3.20-3.66$ & $20-21-25$ & 40.6 & $\begin{array}{l}\text { brown silty clay } \mathrm{w} / \text { pebbles, hard, dry; } \\
\text { wet spot at } 3.35 \mathrm{~m} \text {; moist vertical } \\
\text { fracture at } 3.51 \mathrm{~m}\end{array}$ \\
\hline 1 & 795229 & $3.66-4.12$ & $9-11-15$ & 35.6 & $\begin{array}{l}\text { brown silty clay w/pebbles, soft, } \\
\text { moist, mottled; Fe oxidation }\end{array}$ \\
\hline
\end{tabular}


Table 2.--Test-well construction data and lithologic description of cores at Plot M--Continued

Test well 26--Continued

\begin{tabular}{|c|c|c|c|c|c|}
\hline $\begin{array}{l}\text { Unit } \\
\text { No. }\end{array}$ & $\begin{array}{l}\text { Sample } \\
\text { No. }\end{array}$ & $\begin{array}{c}\text { Depth } \\
\text { interval } \\
\text { (meters) }\end{array}$ & $\begin{array}{l}\text { Blow } \\
\text { counts }\end{array}$ & $\begin{array}{l}\text { Recovery } \\
\quad(\mathrm{cm})\end{array}$ & Description \\
\hline 1 & $79 \$ 230$ & $4.12-4.57$ & $7-7-10$ & 35.6 & $\begin{array}{l}\text { brown silty clay } w / \text { pebbles, soft, } \\
\text { moist, mottled; Fe oxidation }\end{array}$ \\
\hline 1 & 795231 & $4.57-5.03$ & $11-13-13$ & 30.5 & brown silty clay, soft, moist, mottled \\
\hline 1 & $79 S 232$ & $5.03-5.49$ & $9-13-18$ & 50.8 & brown silty clay, soft, moist \\
\hline 1 & 795233 & $5.49-5.94$ & $24-27-27$ & 50.8 & $\begin{array}{l}\text { brown silty clay, soft, moist; } 5.1 \mathrm{~cm} \\
\text { water-bearing sand layer at } 5.94 \mathrm{~m}\end{array}$ \\
\hline 1 & $79 \$ 234$ & $5.94-6.40$ & $13-19-50$ & 35.6 & $\begin{array}{l}\text { brown silty clay; } 30.5 \mathrm{~cm} \text { water-bear- } \\
\text { ing sand layer w/stones at } 6.10 \mathrm{~m}\end{array}$ \\
\hline 1 & $79 \$ 235$ & $6.40-6.86$ & $19-15-16$ & 45.7 & $\begin{array}{l}\text { watery sand to } 6.55 \mathrm{~m} \text {; contact } \mathrm{w} / \text { brown } \\
\text { silty clay } \mathrm{w} / \text { pebbles and stones }\end{array}$ \\
\hline 1 & $79 \$ 236$ & $6.86-7.32$ & $37-32-27$ & 40.6 & brown clayey silt $\mathrm{w} /$ pebbles and stones \\
\hline 1 & 795237 & $7.32-7.77$ & $9-12-17$ & 45.7 & $\begin{array}{l}\text { brown clayey silt grading to brown- } \\
\text { gray clayey silt w/pebbles, stones, } \\
\text { mottled }\end{array}$ \\
\hline 2 & $79 S 238$ & $7.77-8.23$ & $18-20-28$ & 35.6 & $\begin{array}{l}\text { brown-gray clayey silt } w / \text { numerous } \\
\text { pebbles and stones, moist, iron } \\
\text { oxidation }\end{array}$ \\
\hline 2 & 795239 & $8.23-8.69$ & $11-13-18$ & 30.5 & brown-gray clay silt w/pebbles, gritty \\
\hline 2 & $79 S 240$ & $8.69-9.14$ & & NR & \\
\hline 2 & 795241 & $9.14-9.60$ & $25-18-18$ & 30.5 & $\begin{array}{l}\text { brown-gray clayey silt w/pebbles, } \\
\text { gritty }\end{array}$ \\
\hline 2 & $79 S 242$ & $9.60-10.06$ & $12-13-16$ & 40.6 & $\begin{array}{l}\text { brown-gray clayey silt to } 9.75 \mathrm{~m} \text {; } \\
\text { contact dark gray clayey silt w/peb- } \\
\text { bles, dry, hard, dense }\end{array}$ \\
\hline 2 & $79 \$ 243$ & $10.06-10.52$ & $10-15-19$ & 30.5 & $\begin{array}{l}\text { dark gray silt to } 10.21 \mathrm{~m} \text {; contact } \mathrm{w} / \\
\text { brown silt } \mathrm{w} / \text { pebbles and stones }\end{array}$ \\
\hline
\end{tabular}


Table 2.--Test-well construction data and lithologic description of cores at Plot M--Continued

\section{Test well 26--Continued}

\begin{tabular}{|c|c|c|c|c|c|}
\hline $\begin{array}{l}\text { Unit } \\
\text { No. }\end{array}$ & $\begin{array}{l}\text { Sample } \\
\text { No. }\end{array}$ & $\begin{array}{c}\text { Depth } \\
\text { interval } \\
\text { (meters) }\end{array}$ & $\begin{array}{l}\text { Blow } \\
\text { counts }\end{array}$ & $\begin{array}{l}\text { Recovery } \\
\quad(\mathrm{cm})\end{array}$ & Description \\
\hline 2 & 795244 & $10.52-10.97$ & $12-15-17$ & 40.6 & $\begin{array}{l}\text { brown silt w/few pebbles, gritty, } \\
\text { clean }\end{array}$ \\
\hline 2 & $79 S 245$ & $10.97-11.43$ & $39-25-23$ & 30.5 & $\begin{array}{l}\text { brown silt } w / \text { few pebbles, gritty, } \\
\text { moist, dense }\end{array}$ \\
\hline 2 & $79 S 246$ & $11.43-11.89$ & $9-12-16$ & 40.6 & $\begin{array}{l}\text { brown clayey silt w/pebbles, stones, } \\
\text { gritty; } 5.1 \mathrm{~cm} \text { oxidized zone at } \\
11.43 \mathrm{~m}\end{array}$ \\
\hline 2 & $79 S 247$ & $11.89-12.34$ & $12-11-13$ & 25.4 & $\begin{array}{l}\text { gray-brown clayey silt w/pebbles, } \\
\text { stones, gritty, moist, soft }\end{array}$ \\
\hline 2 & 795248 & $12.34-12.80$ & $18-48-12$ & 15.2 & gray-brown clayey silt \\
\hline 2 & $79 \$ 249$ & $12.80-13.26$ & $10-12-45$ & 20.3 & $\begin{array}{l}\text { wet gravelly clayey silt w/pebbles, } \\
\text { stones }\end{array}$ \\
\hline 2 & $79 \$ 250$ & $13.26-13.72$ & $7-8-11$ & 40.6 & $\begin{array}{l}\text { wet graveliy clayey silt grading to } \\
\text { brown-gray clayey silt }\end{array}$ \\
\hline 2 & $79 \$ 251$ & $13.72-14.17$ & $6-11-13$ & 30.5 & $\begin{array}{l}\text { brown-gray clay; grading to pink- } \\
\text { brown silt, moist, clean }\end{array}$ \\
\hline 2 & 795252 & $14.17-14.63$ & $5-7-24$ & 35.6 & $\begin{array}{l}\text { pink-brown silt w/stones, pebbles, } \\
\text { clean }\end{array}$ \\
\hline 2 & 795253 & $14.63-15.09$ & $18-11-23$ & 20.3 & $\begin{array}{l}\text { pink-brown silt to } 14.69 \mathrm{~m} \text {; contact } \\
\text { w/brown silt; graded layers of silt } \\
\text { and sand }\end{array}$ \\
\hline 2 & 795254 & $15.09-15.55$ & $10-14-26$ & 40.6 & $\begin{array}{l}\text { brown clayey silt w/pebbles, gritty, } \\
\text { moist, soft }\end{array}$ \\
\hline 3 & 795255 & $15.55-16.00$ & $26-22-22$ & 40.6 & $\begin{array}{l}\text { brown clayey silt; grading to brown- } \\
\text { gray clayey silt }\end{array}$ \\
\hline 3 & $79 S 256$ & $16.00-16.46$ & $10-20-21$ & 35.6 & $\begin{array}{l}\text { brown-gray clayey silt; grading to } \\
\text { gray-brown clayey silt w/pebbles; } \\
\text { stones }\end{array}$ \\
\hline
\end{tabular}


Table 2.--Test-well construction data and lithologic description of cores at Plot M--Continued

Test well 26--Continued

\begin{tabular}{cccccl}
\hline $\begin{array}{c}\text { Unit } \\
\text { No. }\end{array}$ & $\begin{array}{c}\text { Sample } \\
\text { No. }\end{array}$ & $\begin{array}{c}\text { Depth } \\
\text { interval } \\
\text { (meters) }\end{array}$ & $\begin{array}{c}\text { Blow } \\
\text { counts }\end{array}$ & $\begin{array}{c}\text { Recovery } \\
(\mathrm{cm})\end{array}$ & \multicolumn{1}{c}{ Description } \\
\hline 3 & $79 \mathrm{~s} 257$ & $16.46-16.92$ & $15-25-50 / 4$ & 35.6 & $\begin{array}{l}\text { gray-brown clayey silt; grading to } \\
\text { silt w/pebbles, large stones, soft, } \\
\text { moist }\end{array}$ \\
\hline 3 & $79 \mathrm{~S} 258$ & $16.92-17.37$ & $26-20-20$ & NR & rocks \\
\hline 3 & $79 \mathrm{~S} 259$ & $17.37-17.83$ & $12-16-17$ & 15.2 & $\begin{array}{l}\text { gray-brown clayey silt w/pebbles, } \\
\text { mottled }\end{array}$ \\
\hline 3 & 795260 & $17.83-18.29$ & $19-15-16$ & 30.5 & $\begin{array}{l}\text { brown-gray clayey silt; grading to } \\
\text { gray clayey silt w/pebbles, gritty, } \\
\text { moist, soft }\end{array}$ \\
\hline
\end{tabular}

EOB $19.81 \mathrm{~m}$ 
Table 2.--Test-well construction data and lithologic description of cores at Plot M--Continued

\section{Test well 27}

Completion date: September 27, 1979

Location: 30 meters west of test well 28, 62 meters northwest of the northwest corner of Plot $M$

Site and well construction data: 2 test wells; $18.44 \mathrm{~m}, 24.54 \mathrm{~m}$

Altitude of land surface: $18.44 \mathrm{~m}$ test well. . . . . . $213.21 \mathrm{~m}$ $24.54 \mathrm{~m}$ test well. . . . . . . $213.36 \mathrm{~m}$

Altitude of measuring point (top of casing): $18.44 \mathrm{~m}$ test well. $.212 .91 \mathrm{~m}$ $24.54 \mathrm{~m}$ test well. . $212.84 \mathrm{~m}$

Depth of bottom of piezometer from land surface: $18.44 \mathrm{~m}$ test well. $.18 .74 \mathrm{~m}$ $24.54 \mathrm{~m}$ test well. $25.05 \mathrm{~m}$

Type of piezometer: $1.22 \mathrm{~m}$ slotted PVC pipe, $5.08 \mathrm{~cm}$ diameter

\begin{tabular}{cccccl}
\hline $\begin{array}{c}\text { Unit } \\
\text { No. }\end{array}$ & $\begin{array}{c}\text { Sample } \\
\text { No. }\end{array}$ & $\begin{array}{c}\text { Depth } \\
\text { interval } \\
\text { (meters) }\end{array}$ & $\begin{array}{c}\text { Blow } \\
\text { counts }\end{array}$ & $\begin{array}{c}\text { Recovery } \\
(\mathrm{cm})\end{array}$ & \multicolumn{1}{l}{ Description } \\
\hline 1 & 795106 & $0.15-0.61$ & $9-10-12$ & 10.2 & yellow-brown soil \\
\hline 1 & 795107 & $0.61-1.07$ & $14-15-32$ & 15.2 & \\
\hline 1 & 795108 & $1.07-1.52$ & $20-24-27$ & 20.3 & $\begin{array}{l}\text { brown clayey soil, dry, flaky, root } \\
\text { tubes }\end{array}$ \\
\hline 1 & 795109 & $1.52-1.98$ & $32-37-42$ & 10.2 & brown-gray clayey soil, dry, hard \\
\hline 1 & 795110 & $1.98-2.44$ & $15-19-24$ & 43.2 & $\begin{array}{l}\text { brown clayey soil w/pebbles, stones, } \\
\text { dry, hard; plant debris; Fe oxidation }\end{array}$ \\
\hline 1 & 795111 & $2.44-2.90$ & $15-16-22$ & 45.7 & $\begin{array}{l}\text { brown silty clay w/pebbles, stones, } \\
\text { dry, hard }\end{array}$ \\
\hline 1 & 795112 & $2.90-3.35$ & $22-27-6$ & 35.6 & $\begin{array}{l}\text { brown silty clay w/pebbles, stones; } \\
\text { plant fragments, Fe oxidation }\end{array}$ \\
\hline 1 & 795113 & $3.35-3.81$ & $10-12-16$ & 40.6 & $\begin{array}{l}\text { brown silty clay w/stones to 3.66 m; } \\
3.66 \text { to 3.81 m sand, dry }\end{array}$ \\
\hline
\end{tabular}


Table 2.--Test-well construction data and lithologic description of cores at Plot M--Continued

Test well 27--Continued

\begin{tabular}{|c|c|c|c|c|c|}
\hline $\begin{array}{l}\text { Unit } \\
\text { No. }\end{array}$ & $\begin{array}{l}\text { Sample } \\
\text { No. }\end{array}$ & $\begin{array}{c}\text { Depth } \\
\text { interval } \\
\text { (meters) }\end{array}$ & $\begin{array}{l}\text { Blow } \\
\text { counts }\end{array}$ & $\begin{array}{l}\text { Recovery } \\
\quad(\mathrm{cm})\end{array}$ & Description \\
\hline 1 & $79 S 114$ & $3.81-4.27$ & $16-12-15$ & 30.5 & $\begin{array}{l}2.5 \mathrm{~cm} \text { sand, sand parting at } 3.96 \mathrm{~m} \text {; } \\
\text { brown silty clay w/pebbles, stones, } \\
\text { dense, dry, hard, mottled }\end{array}$ \\
\hline 1 & $79 s 115$ & $4.27-4.72$ & $7-9-11$ & 40.6 & $\begin{array}{l}\text { brown silty clay w/pebbles, stones; } \\
\text { dry to moist, soft; pyrite }\end{array}$ \\
\hline 1 & $79 S 116$ & $4.72-5.18$ & $9-12-13$ & 35.6 & $\begin{array}{l}\text { brown silty clay w/pebbles, stones; } \\
\text { dry to moist }\end{array}$ \\
\hline 1 & $79 S 117$ & $5.18-5.64$ & $9-12-21$ & 35.6 & $\begin{array}{l}\text { brown silty clay w/pebbles, stones, } \\
\text { dry, hard }\end{array}$ \\
\hline 1 & 795118 & $5.64-6.10$ & $12-14-15$ & 10.2 & brown silty clay, dry, hard, pyrite \\
\hline 1 & $79 S 119$ & $6.10-6.55$ & $22-24-26$ & 10.2 & brown silty clay w/stones, dry, hard \\
\hline 1 & $79 S 120$ & $6.55-7.01$ & $14-16-24$ & 50.8 & $\begin{array}{l}\text { brown silty clay w/pebbles, stones, } \\
\text { dry, hard }\end{array}$ \\
\hline 1 & $79 S 121$ & $7.01-7.47$ & $12-10-12$ & 45.7 & $\begin{array}{l}\text { brown silty clay to } 7.16 \mathrm{~m} \text {; then wet, } \\
\text { light brown clay w/sand partings to } \\
7.32 \mathrm{~m} \text {; then moist light brown clayey } \\
\text { silt w/pebbles }\end{array}$ \\
\hline 1 & $79 S 122$ & $7.47-7.93$ & $12-30-15$ & 50.8 & $\begin{array}{l}\text { brown clayey silt w/pebbles, large } \\
\text { stones; dry to moist }\end{array}$ \\
\hline 1 & $79 S 123$ & $7.93-8.38$ & $17-22-26$ & 35.6 & $\begin{array}{l}\text { brown silty clay, moist to } 8.08 \mathrm{~m} \text {; } \\
\text { contact } \mathrm{w} / \mathrm{brown} \text { clayey silt } \mathrm{w} / \text { pebbles; } \\
\text { limestone stones }\end{array}$ \\
\hline 1 & $79 S 124$ & $8.38-8.84$ & $37-49-48$ & NR & rocks \\
\hline 1 & $79 S 125$ & $8.84-9.30$ & $17-19-23$ & 61.0 & $\begin{array}{l}\text { brown clayey silt w/oxidized pebbles } \\
\text { to } 9.14 \mathrm{~m} \text {; contact } \mathrm{w} / \text { brown-gray clayey } \\
\text { silt w/oxidized stones and pebbles, } \\
\text { dense, mottled; dry to moist }\end{array}$ \\
\hline
\end{tabular}


Table 2.--Test-well construction data and lithologic description of cores at Plot M--Continued

Test well 27--Continued

\begin{tabular}{cccccl}
\hline $\begin{array}{l}\text { Unit } \\
\text { No. }\end{array}$ & $\begin{array}{c}\text { Sample } \\
\text { No. }\end{array}$ & $\begin{array}{c}\text { Depth } \\
\text { interval } \\
\text { (meters) }\end{array}$ & $\begin{array}{c}\text { Blow } \\
\text { counts }\end{array}$ & $\begin{array}{c}\text { Recovery } \\
(\mathrm{cm})\end{array}$ & Description \\
\hline 2 & 795126 & $9.30-9.75$ & $58-50 / 3$ & NR & (rock in auger) \\
\hline 2 & 795127 & $9.75-10.21$ & $12-13-17$ & 61.0 & $\begin{array}{l}\text { brown gray clayey silt to } 9.91 \mathrm{~m} ; \\
\text { contact w/pink-brown clayey silt w/ } \\
\text { pebbles to 10.10 m; contact w/brown- } \\
\text { gray clayey silt w/pebbles, stones; } \\
\text { grading to gray clayey silt, moist }\end{array}$ \\
\hline 2 & 795128 & $10.21-10.67$ & $21-24-15$ & 30.5 & $\begin{array}{l}\text { gray-brown clayey silt w/pebbles; dry } \\
\text { to moist }\end{array}$ \\
\hline
\end{tabular}

\begin{tabular}{|c|c|c|c|c|c|}
\hline 2 & $79 S 129$ & $10.67-11.13$ & $10-13-15$ & 35.6 & $\begin{array}{l}\text { gray-brown clayey silt } w / \text { pebbles, } \\
\text { stones; dry to moist }\end{array}$ \\
\hline 2 & $79 S 130$ & $11.13-11.58$ & $100-20-12$ & 20.3 & $\begin{array}{l}\text { gray clayey silt w/few pebbles, moist, } \\
\text { soft }\end{array}$ \\
\hline 2 & 795131 & $11.58-12.04$ & $24-17-23$ & 10.2 & $\begin{array}{l}\text { gray clayey silt w/few pebbles, moist, } \\
\text { soft }\end{array}$ \\
\hline 2 & $79 S 132$ & $12.04-12.50$ & $14-19-18$ & 40.6 & $\begin{array}{l}\text { gray-brown clayey silt } w / \text { numerous } \\
\text { pebbles, stones, moist, soft }\end{array}$ \\
\hline 2 & $79 S 133$ & $12.50-12.95$ & $10-16-18$ & 61.0 & $\begin{array}{l}\text { brown clayey silt w/pebbles, stones } \\
\text { to } 12.65 \mathrm{~m} \text {; contact w/gray silt w/ } \\
\text { pebbles }\end{array}$ \\
\hline 2 & $79 S 134$ & $12.95-13.41$ & $18-15-16$ & 50.8 & $\begin{array}{l}\text { brown-gray clayey silt } w / \text { pebbles, } \\
\text { dense, hard, dry to moist }\end{array}$ \\
\hline 2 & $79 S 135$ & $13.41-13.87$ & $16-22-25$ & 50.8 & $\begin{array}{l}\text { brown-gray clayey silt w/pebbles, } \\
\text { dense, hard, moist }\end{array}$ \\
\hline 2 & $79 S 136$ & $13.87-14.35$ & $45-37-50 / 5$ & 40.6 & $\begin{array}{l}\text { brown-gray clayey silt } w / \text { pebbles to } \\
14.17 \mathrm{~m} \text {; contact } w / 15.3 \mathrm{~cm} \mathrm{dry,} \\
\text { yellow sand }\end{array}$ \\
\hline 2 & 795137 & $14.35-14.78$ & $100 / 3$ & 15.2 & $\begin{array}{l}\text { gray clayey silt to } 14.48 \mathrm{~m} ; 5.1 \mathrm{~cm} \\
\text { sand layer brown-gray clayey silt w/ } \\
\text { pebbles to } 14.81 \mathrm{~m} \text {; brown }\end{array}$ \\
\hline
\end{tabular}


Table 2.--Test-well construction data and lithologic description of cores at Plot M--Continued

\section{Test well 27--Continued}

\begin{tabular}{|c|c|c|c|c|c|}
\hline $\begin{array}{l}\text { Unit } \\
\text { No. }\end{array}$ & $\begin{array}{l}\text { Sample } \\
\text { No. }\end{array}$ & $\begin{array}{c}\text { Depth } \\
\text { interval } \\
\text { (meters) }\end{array}$ & $\begin{array}{l}\text { Blow } \\
\text { counts }\end{array}$ & $\begin{array}{l}\text { Recovery } \\
\quad(\mathrm{cm})\end{array}$ & Description \\
\hline 2 & $79 \$ 138$ & $14.78-15.24$ & $10-12-15$ & 50.8 & $\begin{array}{l}\text { gray clayey silt; grading downwards } \\
\text { to brown-gray clayey silt, moist, soft }\end{array}$ \\
\hline 2 & 795139 & $15.24-15.70$ & $9-11-17$ & 25.4 & $\begin{array}{l}\text { brown-gray clayey silt; soft, moist; } \\
\text { contact w/gray clayey silt }\end{array}$ \\
\hline 2 & 795140 & $15.70-16.15$ & $16-19-27$ & 30.5 & $\begin{array}{l}\text { gray clayey silt w/few pebbles, soft } \\
\text { w/buff bleach zone at } 15.73 \mathrm{~m}\end{array}$ \\
\hline 2 & $79 S 141$ & $16.15-16.61$ & $20-18-21$ & 61.0 & $\begin{array}{l}\text { brown-gray silt; grading downward to } \\
\text { gray clayey silt w/pebbles, stones }\end{array}$ \\
\hline 2 & $79 S 142$ & $16.61-17.07$ & $10-16-18$ & 61.0 & $\begin{array}{l}\text { gray clayey silt w/pebbles, stones, } \\
\text { moist, soft }\end{array}$ \\
\hline 2 & $79 S 143$ & $17.07-17.52$ & $16-18-26$ & 40.6 & $\begin{array}{l}\text { gray clayey silt w/few pebbles, dry } \\
\text { to moist, soft }\end{array}$ \\
\hline 2 & $79 S 144$ & $17.52-17.98$ & $17-19-43$ & 20.3 & $\begin{array}{l}\text { gray clayey silt w/pebbles to } 17.62 \mathrm{~m} \text {; } \\
\text { contact } 5.1 \mathrm{~cm} \text { yellow, oxidized, sand } \\
\text { (sharp, clear, red } \mathrm{Fe} \text { oxidation) }\end{array}$ \\
\hline 2 & $79 S 145$ & $17.98-18.44$ & $26-33-36$ & 30.5 & $\begin{array}{l}\text { gray clayey silt w/stones; yellow } \\
\text { sand parting at } 18.01 \mathrm{~m}\end{array}$ \\
\hline 2 & $79 S 146$ & $18.44-18.90$ & $11-13-10$ & 40.6 & $\begin{array}{l}\text { gray clayey silt w/pebbles, stones, } \\
\text { moist, soft }\end{array}$ \\
\hline 2 & $79 S 147$ & $18.90-19.35$ & $38-26-21$ & 30.5 & $\begin{array}{l}\text { gray clayey silt w/pebbles, stones to } \\
19.05 \mathrm{~m} \text {; contact } \mathrm{w} / \text { gray clayey silt } \\
\text { w/few pebbles or stones, gritty, moist }\end{array}$ \\
\hline 2 & $79 \$ 148$ & $19.35-19.81$ & $43-26-24$ & 35.6 & $\begin{array}{l}\text { grayish brown clayey silt; } 2.8 \mathrm{~cm} \text {, } \\
\text { lenses of broken rock, sand, gravel } \\
\text { near } 19.66 \mathrm{~m}\end{array}$ \\
\hline 3 & $79 S 149$ & $19.81-20.27$ & $22-18-24$ & 30.5 & gray clayey silt $\mathrm{w} /$ pebbles, moist \\
\hline 3 & $79 \$ 150$ & $20.27-20.73$ & $21-26-23$ & 30.5 & $\begin{array}{l}\text { gray clayey silt } w / \text { pebbles, stones; } \\
\text { dry to moist }\end{array}$ \\
\hline
\end{tabular}


Table 2.--Test-well construction data and lithologic description of cores at Plot M--Continued

Test well 27--Continued

\begin{tabular}{|c|c|c|c|c|c|}
\hline $\begin{array}{l}\text { Unit } \\
\text { No. }\end{array}$ & $\begin{array}{l}\text { Sample } \\
\text { No. }\end{array}$ & $\begin{array}{c}\text { Depth } \\
\text { interval } \\
\text { (meters) }\end{array}$ & $\begin{array}{l}\text { Blow } \\
\text { counts }\end{array}$ & $\begin{array}{l}\text { Recovery } \\
\quad(\mathrm{cm})\end{array}$ & Description \\
\hline 3 & $79 S 151$ & $20.73-21.18$ & $14-18-24$ & 35.6 & $\begin{array}{l}\text { gray clayey silt } w / \text { pebbles, stones; } \\
\text { dry to moist }\end{array}$ \\
\hline 3 & $79 S 152$ & $21.18-21.64$ & $16-20-20$ & 10.6 & $\begin{array}{l}\text { gray clayey silt } w / \text { pebbles, stones; } \\
\text { dry to moist }\end{array}$ \\
\hline 3 & $79 S 153$ & $21.64-22.10$ & $21-21-26$ & 40.6 & $\begin{array}{l}\text { gray clayey silt } w / \text { pebbles; dry to } \\
\text { moist, brittle }\end{array}$ \\
\hline 3 & $79 S 154$ & $22.10-22.56$ & $35-31-26$ & 50.8 & $\begin{array}{l}\text { gray clayey silt w/pebbles; dry to } \\
\text { moist, brittle }\end{array}$ \\
\hline 3 & $79 S 155$ & $22.56-23.01$ & $35-32-23$ & 35.6 & gray clayey silt w/pebbles, dry \\
\hline 3 & $79 S 156$ & $23.01-23.47$ & $17-24-25$ & 45.7 & $\begin{array}{l}\text { gray clayey silt } w / \text { few pebbles, dry, } \\
\text { brittle }\end{array}$ \\
\hline 3 & 795157 & $23.47-23.93$ & $24-25-31$ & 45.7 & $\begin{array}{l}\text { gray clayey silt } w / \text { few pebbles; dry } \\
\text { to moist }\end{array}$ \\
\hline 3 & $79 S 158$ & $23.93-24.38$ & $20-23-23$ & 30.5 & $\begin{array}{l}\text { gray clayey silt } w / \text { few pebbles; dry } \\
\text { to moist }\end{array}$ \\
\hline
\end{tabular}


Table 2.--Test-well construction data and lithologic description of cores at Plot M--Continued

\section{Test well 28}

\section{Completion date: October 3, 1979}

Location: 65 meters north-northwest of the northwest corner of Plot M Site and well construction data: 2 test wells; $18.44 \mathrm{~m}, 24.54 \mathrm{~m}$

Altitude of land surface: $18.44 \mathrm{~m}$ test well. . . . . . . $208.54 \mathrm{~m}$ $24.54 \mathrm{~m}$ test well. . . . . . . $208.63 \mathrm{~m}$

Altitude of measuring point (top of casing): $18.44 \mathrm{~m}$ test well. $.208 .73 \mathrm{~m}$ $24.54 \mathrm{~m}$ test well. . $208.79 \mathrm{~m}$

Depth to bottom of piezometer from land surface: $18.44 \mathrm{~m}$ test well. $.18 .25 \mathrm{~m}$ $24.54 \mathrm{~m}$ test well. $24.38 \mathrm{~m}$

Type of piezometer: $1.22 \mathrm{~m}$ slotted PVC pipe, $5.08 \mathrm{~cm}$ diamater

\begin{tabular}{|c|c|c|c|c|c|}
\hline $\begin{array}{l}\text { Unit } \\
\text { No. }\end{array}$ & $\begin{array}{l}\text { Sample } \\
\text { No. }\end{array}$ & $\begin{array}{c}\text { Depth } \\
\text { interval } \\
\text { (meters) }\end{array}$ & $\begin{array}{l}\text { Blow } \\
\text { counts }\end{array}$ & $\begin{array}{l}\text { Recovery } \\
\quad(\mathrm{cm})\end{array}$ & Description \\
\hline 1 & 7951 & $0.31-0.76$ & $5-4-5$ & 29.2 & brown silty clay, dry, hard, mottled \\
\hline 1 & 7952 & $0.76-1.22$ & $8-10-11$ & 35.6 & brown silty clay, dry, hard, mottled \\
\hline 1 & 7953 & $1.22-1.66$ & $8-10-11$ & 45.7 & $\begin{array}{l}\text { brown-gray silty clay w/pebbles, dry, } \\
\text { hard; Fe oxidation }\end{array}$ \\
\hline 1 & 7954 & $1.66-2.13$ & $7-11-13$ & 48.3 & $\begin{array}{l}\text { brown-gray silty clay w/pebbles, dry, } \\
\text { hard; Fe oxidation; } 2.5 \mathrm{~cm} \text { wet zone } \\
\text { at } 1.83 \mathrm{~m}\end{array}$ \\
\hline 1 & 7955 & $2.13-2.59$ & $10-14-18$ & 50.8 & $\begin{array}{l}\text { brown-gray silty clay, dense, hard, } \\
\text { mottled; zone of wetness at } 2.29 \mathrm{~m}\end{array}$ \\
\hline 1 & 7956 & $2.59-3.05$ & $11-13-15$ & 50.8 & $\begin{array}{l}\text { brown-gray silty clay w/pebbles, } \\
\text { dense, hard, mottled }\end{array}$ \\
\hline 1 & 7957 & $3.05-3.51$ & $10-15-14$ & 50.8 & $\begin{array}{l}\text { brown-gray silty clay w/pebbles, } \\
\text { dense, hard; Fe oxidation stains, } \\
\text { pyrite }\end{array}$ \\
\hline 1 & 7958 & $3.51-3.96$ & $9-14-14$ & 50.8 & $\begin{array}{l}\text { brown silty clay w/pebbles; } 7.6 \mathrm{~cm} \\
\text { sand lense at } 4.11 \mathrm{~m}\end{array}$ \\
\hline
\end{tabular}


Table 2.--Test-well construction data and lithologic description of cores at Plot M--Continued

Test well 28--Continued

\begin{tabular}{|c|c|c|c|c|c|}
\hline $\begin{array}{l}\text { Unit } \\
\text { No. }\end{array}$ & $\begin{array}{c}\text { Sample } \\
\text { No. }\end{array}$ & $\begin{array}{c}\text { Depth } \\
\text { interval } \\
\text { (meters) }\end{array}$ & $\begin{array}{l}\text { Blow } \\
\text { counts }\end{array}$ & $\begin{array}{l}\text { Recovery } \\
(\mathrm{cm})\end{array}$ & Description \\
\hline 1 & 7959 & $3.96-4.42$ & $10-15-17$ & 61.0 & $\begin{array}{l}\text { brown silty clay w/pebbles, stones, } \\
\text { hard, dense; Fe oxidation }\end{array}$ \\
\hline 1 & $79 S 10$ & $4.42-4.88$ & $9-14-17$ & 55.9 & $\begin{array}{l}\text { brown silty clay w/pebbles, hard, } \\
\text { dense; Fe oxidation }\end{array}$ \\
\hline 1 & 79511 & $4.88-5.33$ & $16-22-19$ & 55.9 & $\begin{array}{l}\text { brown silty clay w/pebbles, stones; } \\
\text { Fe oxidation; } 3 \text { sand partings near } \\
5.33 \mathrm{~m}\end{array}$ \\
\hline 1 & $79 S 12$ & $5.33-5.79$ & $20-20-18$ & 38.1 & $\begin{array}{l}\text { brown muddy sand w/pebbles; water- } \\
\text { bearing sand layer from } 5.33 \text { to } 5.50 \mathrm{~m}\end{array}$ \\
\hline 1 & $79 S 13$ & $5.79-6.25$ & $6-11-14$ & 45.7 & $\begin{array}{l}\text { brown silty clay w/pebbles, stones, } \\
\text { dense; Fe oxidation; } 5.1 \mathrm{~cm} \text { water- } \\
\text { bearing sand lenses at } 5.79 \text { and } 6.10 \mathrm{~m}\end{array}$ \\
\hline 1 & 79514 & $6.25-6.70$ & $21-24-26$ & 30.5 & $\begin{array}{l}\text { brown silty clay w/pebbles, Fe oxida- } \\
\text { tion; } 5.08 \mathrm{~cm} \text { water-bearing sand } \\
\text { lense at } 6.71 \mathrm{~m}\end{array}$ \\
\hline 1 & $79 S 15$ & $6.70-7.16$ & $14-18-22$ & NR & \\
\hline 1 & 79516 & $7.16-7.60$ & $14-50$ & NR & (augered $0.9 \mathrm{~m}$ to get past large rock) \\
\hline 1 & & $7.60-8.08$ & & & \\
\hline 2 & $79 S 17$ & $8.08-8.53$ & $17-19-19$ & 61.0 & $\begin{array}{l}\text { brown silty clay to } 8.23 \mathrm{~m} \text {; contact } \\
\text { w/light brown silty clay to } 8.38 \mathrm{~m} \text {; } \\
\text { contact w/gray clay }\end{array}$ \\
\hline 2 & 79518 & $8.53-8.99$ & $14-18-26$ & 55.9 & $\begin{array}{l}\text { gray silty clay w/limestone pebbles; } \\
\text { stones, dense, moist }\end{array}$ \\
\hline 2 & 79519 & $8.99-9.45$ & $22-24-28$ & 61.0 & $\begin{array}{l}\text { gray silty clay w/limestone pebbles, } \\
\text { stones, gravel, Fe oxidation }\end{array}$ \\
\hline 2 & $79 s 20$ & $9.45-9.90$ & $22-22-29$ & NR & \\
\hline
\end{tabular}


Table 2.--Test-well construction data and lithologic description of cores at Plot M--Continued

Test well 28--Continued

\begin{tabular}{|c|c|c|c|c|c|}
\hline $\begin{array}{l}\text { Unit } \\
\text { No. }\end{array}$ & $\begin{array}{l}\text { Sample } \\
\text { No. }\end{array}$ & $\begin{array}{c}\text { Depth } \\
\text { interval } \\
\text { (meters) }\end{array}$ & $\begin{array}{l}\text { Blow } \\
\text { counts }\end{array}$ & $\begin{array}{l}\text { Recovery } \\
\quad(\mathrm{cm})\end{array}$ & Description \\
\hline 2 & 79521 & $9.90-10.36$ & $20-14-19$ & 35.6 & $\begin{array}{l}\text { gray clayey silt w/pebbles, stones, } \\
\text { dense }\end{array}$ \\
\hline 2 & 79522 & $10.36-10.82$ & $15-14-25$ & 40.6 & dark gray clayey silt w/pebbles \\
\hline 2 & 79523 & $10.82-11.28$ & $24-36-24$ & 45.7 & dark gray clayey silt $\mathrm{w} /$ pebbles, moist \\
\hline 2 & 79524 & $11.28-11.73$ & $20-19-14$ & 35.6 & $\begin{array}{l}\text { dark gray clayey silt } \mathrm{w} / \mathrm{sand} \text {, pebbles, } \\
\text { stones, moist }\end{array}$ \\
\hline 2 & $79 S 25$ & $11.73-12.19$ & $7-12-17$ & 61.0 & $\begin{array}{l}\text { dark gray clayey silt w/pebbles, } \\
\text { stones, moist }\end{array}$ \\
\hline 2 & $79 s 26$ & $12.19-12.65$ & $18-12-17$ & 45.7 & $\begin{array}{l}\text { gray clayey silt w/sand, pebbles, } \\
\text { stones, dense }\end{array}$ \\
\hline 2 & 79527 & $12.65-13.10$ & $8-13-13$ & 55.9 & $\begin{array}{l}\text { brown-gray clayey silt w/sand, peb- } \\
\text { bles, Fe oxidation; grading to gray } \\
\text { clayey silt; horizontal fracture at } \\
12.80 \mathrm{~m} ; 2.5 \mathrm{~cm} \text { sand lense at } 12.80 \mathrm{~m}\end{array}$ \\
\hline 2 & 79528 & $13.10-13.56$ & $8-10-12$ & 61.0 & gray clayey silt w/pebbles, dense \\
\hline 2 & $79 \$ 29$ & $13.56-14.02$ & $9-12-17$ & 45.7 & $\begin{array}{l}\text { gray clayey silt w/pebbles, stones; } \\
\text { wet sandy clayey silt at } 13.56 \mathrm{~m}\end{array}$ \\
\hline 2 & 79530 & $14.02-14.48$ & $32-13-17$ & 45.7 & $\begin{array}{l}\text { sandy clayey silt } 14.02 \text { to } 14.17 \mathrm{~m} \text {; } \\
\text { gray clayey silt w/pebbles }\end{array}$ \\
\hline 2 & 79531 & $14.48-14.94$ & $12-15-20$ & 30.5 & $\begin{array}{l}5.1 \mathrm{~cm} \text { wet sand lense at } 14.48 \mathrm{~m} \text {; } \\
\text { gray clayey silt w/pebbles, Fe oxida- } \\
\text { tion }\end{array}$ \\
\hline 2 & $79 \mathrm{~S} 32$ & $14.94-15.39$ & $10-14-16$ & 61.0 & $\begin{array}{l}\text { clayey silt w/pebbles and Fe oxida- } \\
\text { tion; grading to more dense silts; } \\
\text { horizontal fracture at } 15.00 \mathrm{~m}\end{array}$ \\
\hline 2 & 79533 & $15.39-15.85$ & $8-10-18$ & 35.6 & gray clayey silt, moist, dense \\
\hline
\end{tabular}


Table 2.--Test-well construction data and lithologic description of cores at Plot M--Continued

Test well 28--Continued

\begin{tabular}{|c|c|c|c|c|c|}
\hline $\begin{array}{l}\text { Unit } \\
\text { No. }\end{array}$ & $\begin{array}{l}\text { Sample } \\
\text { No. }\end{array}$ & $\begin{array}{c}\text { Depth } \\
\text { interval } \\
\text { (meters) }\end{array}$ & $\begin{array}{l}\text { Blow } \\
\text { counts }\end{array}$ & $\begin{array}{l}\text { Recovery } \\
\quad(\mathrm{cm})\end{array}$ & Description \\
\hline 2 & $79 S 34$ & $15.85-16.31$ & $13-16-24$ & 61.0 & $\begin{array}{l}\text { gray clayey silt w/pebbles, Fe oxida- } \\
\text { tion }\end{array}$ \\
\hline 2 & $79 S 35$ & $16.31-16.76$ & $38-25-26$ & 61.0 & $\begin{array}{l}\text { gray clayey silt w/pebbles, gritty; } \\
\text { dry, moist }\end{array}$ \\
\hline 3 & $79 \$ 36$ & $16.76-17.22$ & $17-23-21$ & 50.8 & $\begin{array}{l}10.12 \mathrm{~cm} \text { water-bearing sand layer at } \\
16.76 \mathrm{~m} \text {; gray clayey silt w/pebbles, } \\
\text { stones, moist, dense }\end{array}$ \\
\hline 3 & 79537 & $17.22-17.68$ & $54-35-34$ & 35.6 & gray clayey silt w/pebbles, mottled \\
\hline 3 & 79538 & $17.68-18.14$ & $32-34-50 / 5$ & NR & \\
\hline 3 & 79539 & $18.14-18.59$ & $32-35-49$ & 25.4 & gray clayey silt w/pebbles, moist \\
\hline 3 & $79 S 40$ & $18.59-19.05$ & $22-31-40$ & 45.7 & $\begin{array}{l}\text { gray clayey silt } w / \text { pebbles; dry to } \\
\text { moist }\end{array}$ \\
\hline 3 & $79 S 41$ & $19.05-19.51$ & $17-23-30$ & 33.0 & $\begin{array}{l}\text { gray clayey silt w/limestone pebbles, } \\
\text { moist, hard }\end{array}$ \\
\hline 3 & $79 S 42$ & $19.51-19.96$ & $37-32-39$ & 50.8 & $\begin{array}{l}\text { gray clayey silt w/few pebbles; dry } \\
\text { to moist }\end{array}$ \\
\hline 3 & $79 S 43$ & $19.96-20.42$ & $20-40-50 / 5$ & NR & \\
\hline 3 & $79 S 44$ & $20.42-20.88$ & $22-29-36$ & 50.8 & $\begin{array}{l}\text { gray clayey silt } \mathrm{w} / \text { pebbles, stones, } \\
\text { moist, dense; } 2 \text { sand partings at } \\
21.03 \mathrm{~m}\end{array}$ \\
\hline 3 & $79 S 45$ & $20.88-21.33$ & $25-34-35$ & 10.1 & \\
\hline 3 & $79 S 46$ & $21.33-21.79$ & $25-34-50$ & 15.2 & gray clayey silt \\
\hline 3 & $79 \$ 47$ & $21.79-22.25$ & $41-31-53$ & 25.4 & $\begin{array}{l}\text { gray clayey silt w/pebbles, stones, } \\
\text { moist, dense }\end{array}$ \\
\hline
\end{tabular}

$3 \quad 79 S 48 \quad 22.25-22.71 \quad 25-33-50 / 5 \quad$ NR 
Table 2.--Test-well construction data and lithologic description of cores at Plot M--Continued

Test well 28--Continued

\begin{tabular}{cccccl}
\hline $\begin{array}{l}\text { Unit } \\
\text { No. }\end{array}$ & $\begin{array}{c}\text { Sample } \\
\text { No. }\end{array}$ & $\begin{array}{c}\text { Depth } \\
\text { interval } \\
\text { (meters) }\end{array}$ & $\begin{array}{c}\text { Blow } \\
\text { counts }\end{array}$ & $\begin{array}{c}\text { Recovery } \\
(\mathrm{cm})\end{array}$ & \multicolumn{1}{c}{ Description } \\
\hline 4 & 79549 & $22.71-23.16$ & $46-36-34$ & 50.8 & $\begin{array}{l}\text { gray clayey silt w/pebbles, stones, } \\
\text { moist, mottled }\end{array}$ \\
\hline 4 & 79550 & $23.16-23.62$ & $52-32-32$ & 25.4 & $\begin{array}{l}\text { gray clayey silt w/pebbles, very hard; } \\
\text { dry to moist, mottled }\end{array}$ \\
\hline 4 & 79551 & $23.62-24.08$ & $30-39-47$ & 35.6 & $\begin{array}{l}\text { gray clayey silt w/pebbles, stones, } \\
\text { hard, dry }\end{array}$ \\
\hline 4 & 79552 & $24.08-24.53$ & $24-31-35$ & 50.8 & $\begin{array}{l}\text { gray clayey silt w/few pebbles, hard, } \\
\text { dry }\end{array}$ \\
\hline
\end{tabular}

EOB $24.53 \mathrm{~m}$ 
Table 2.--Test-well construction data and lithologic description of cores at Plot M--Continued

\section{Test well 29}

Completion date: September 25, 1979

Location: 83 meters north-northwest of the center of Plot M, 30 meters west of test well 5

Site and well construction data: 2 test wells; $18.44 \mathrm{~m}, 24.54 \mathrm{~m}$

Altitude of land surface: $18.44 \mathrm{~m}$ test well. . . . . . $206.50 \mathrm{~m}$

$24.54 \mathrm{~m}$ test well. . . . . . . $206.50 \mathrm{~m}$

Altitude of measuring point (top of casing): $18.44 \mathrm{~m}$ test well. . $206.62 \mathrm{~m}$

$24.54 \mathrm{~m}$ test well. . $206.62 \mathrm{~m}$

Depth of bottom of piezometer from land surface: $18.44 \mathrm{~m}$ test well. $18.32 \mathrm{~m}$

$24.54 \mathrm{~m}$ test well. $24.42 \mathrm{~m}$

Type of piezometer: $1.22 \mathrm{~m}$ slotted PVC pipe, $5.08 \mathrm{~cm}$ diameter

\begin{tabular}{|c|c|c|c|c|c|}
\hline $\begin{array}{l}\text { Unit } \\
\text { No. }\end{array}$ & $\begin{array}{l}\text { Sample } \\
\text { No. }\end{array}$ & $\begin{array}{c}\text { Depth } \\
\text { interval } \\
\text { (meters) }\end{array}$ & $\begin{array}{l}\text { Blow } \\
\text { counts }\end{array}$ & $\begin{array}{l}\text { Recovery } \\
\quad(\mathrm{cm})\end{array}$ & Description \\
\hline 1 & 79553 & $0.15-0.61$ & $6-6-5$ & 22.9 & $\begin{array}{l}\text { brown silty clay soil w/stones, dry, } \\
\text { weathered, brittle, plant fragments }\end{array}$ \\
\hline 1 & 79554 & $0.61-1.07$ & $4-6-10$ & 22.9 & $\begin{array}{l}\text { brown clay soil w/stones, dry, tree } \\
\text { stems, root tubes }\end{array}$ \\
\hline 1 & 79555 & $1.07-1.52$ & $23-20-21$ & 25.4 & $\begin{array}{l}\text { brown clay soil w/pebbles, stones, } \\
\text { dry }\end{array}$ \\
\hline 1 & $79 S 56$ & $1.52-1.98$ & $16-22-18$ & NR & \\
\hline 1 & 79557 & $1.98-2.44$ & $14-19-20$ & 20.3 & $\begin{array}{l}\text { brown clayey soil w/plant fragments, } \\
\text { dry, hard; } 20.3 \mathrm{~cm} \text { vertical fracture } \\
\text { sand coated at } 1.98 \mathrm{~m} \text {; three } 2.5 \mathrm{~cm} \\
\text { dry sand lenses at } 2.29 \mathrm{~m}\end{array}$ \\
\hline 1 & 79558 & $2.44-2.90$ & $10-18-17$ & 61.0 & $\begin{array}{l}25.4 \mathrm{~cm} \text { water-bearing sand layer at } \\
2.44 \mathrm{~m} \text {; contact w/brown silty clay } \\
\text { w/pebbles }\end{array}$ \\
\hline 1 & 79559 & $2.90-3.35$ & $9-14-17$ & 35.6 & $\begin{array}{l}\text { brown silty clay w/Fe oxidized peb- } \\
\text { bles, stones, moist, hard }\end{array}$ \\
\hline
\end{tabular}


Table 2.--Test-well construction data and lithologic description of cores at Plot $\mathrm{M}$--Continued

Test well 29--Continued

\begin{tabular}{|c|c|c|c|c|c|}
\hline $\begin{array}{l}\text { Unit } \\
\text { No. }\end{array}$ & $\begin{array}{l}\text { Sample } \\
\text { No. }\end{array}$ & $\begin{array}{c}\text { Depth } \\
\text { interval } \\
\text { (meters) }\end{array}$ & $\begin{array}{l}\text { Blow } \\
\text { counts }\end{array}$ & $\begin{array}{l}\text { Recovery } \\
\quad(\mathrm{cm})\end{array}$ & Description \\
\hline 1 & 79560 & $3.35-3.81$ & $47-23-35$ & 40.6 & $\begin{array}{l}\text { brown silty clay w/oxidized pebbles, } \\
\text { stones, plant fragments, moist, very } \\
\text { hard }\end{array}$ \\
\hline 1 & $79 S 61$ & $3.81-4.27$ & $11-14-19$ & 66.0 & $\begin{array}{l}\text { brown silty clay w/numerous pebbles, } \\
\text { stones, mottled, oxidized zones, } \\
\text { dense, very hard }\end{array}$ \\
\hline 1 & $79 S 62$ & $4.27-4.72$ & $12-17-21$ & 45.7 & $\begin{array}{l}\text { brown silty clay w/large pebbles, } \\
\text { stones }\end{array}$ \\
\hline 1 & 79563 & $4.72-5.18$ & $9-14-16$ & 45.7 & $\begin{array}{l}\text { brown-gray silty clay w/pebbles, } \\
\text { stones, plant fragments, moist }\end{array}$ \\
\hline 2 & $79 S 64$ & $5.18-5.64$ & $13-17-19$ & 35.6 & $\begin{array}{l}\text { brown-gray silty clay } w / \text { pebbles, } \\
\text { stones, moist }\end{array}$ \\
\hline 2 & $79 S 65$ & $5.64-6.10$ & $5-5-9$ & NR & \\
\hline 2 & 79566 & $6.10-6.55$ & $11-16-16$ & 40.6 & $\begin{array}{l}\text { brown gray silty clay to } 6.25 \mathrm{~m} \text {; } \\
\text { grading to gray silty clay } \mathrm{w} / \text { pebbles, } \\
\text { stones }\end{array}$ \\
\hline 2 & 79567 & $6.55-7.01$ & $12-24-24$ & 40.6 & $\begin{array}{l}\text { gray silty clay w/pebbles; } 10.2 \mathrm{~cm} \\
\text { oxidized zone, brown at } 6.71 \mathrm{~m}\end{array}$ \\
\hline 2 & 79568 & $7.01-7.47$ & $10-12-17$ & 40.6 & $\begin{array}{l}\text { gray-brown clayey silt w/pebbles, } \\
\text { stones, plant fragments, mottling, } \\
\text { moist, soft }\end{array}$ \\
\hline 2 & $79 S 69$ & $7.47-7.93$ & $12-16-20$ & 45.7 & $\begin{array}{l}\text { gray-brown clayey silt w/pebbles, } \\
\text { moist, dense }\end{array}$ \\
\hline 2 & 79570 & $7.93-8.38$ & $9-50 / 3$ & 35.1 & $\begin{array}{l}\text { gray clayey silt } \mathrm{w} / \text { pebbles, stones, } \\
\text { oxidized, mottled }\end{array}$ \\
\hline 2 & $79 S 71$ & $8.38-8.84$ & $25-18-14$ & 45.7 & $\begin{array}{l}\text { dark gray clayey silt w/numerous peb- } \\
\text { bles, stones, very hard }\end{array}$ \\
\hline
\end{tabular}


Table 2.--Test-well construction data and lithologic description of cores at Plot $\mathrm{M}$--Continued

Test well 29--Continued

\begin{tabular}{|c|c|c|c|c|c|}
\hline $\begin{array}{l}\text { Unit } \\
\text { No. }\end{array}$ & $\begin{array}{c}\text { Sample } \\
\text { No. }\end{array}$ & $\begin{array}{c}\text { Depth } \\
\text { interval } \\
\text { (meters) } \\
\end{array}$ & $\begin{array}{l}\text { Blow } \\
\text { counts }\end{array}$ & $\begin{array}{l}\text { Recovery } \\
(\mathrm{cm})\end{array}$ & Description \\
\hline 2 & 79572 & $8.84-9.30$ & $7-12-14$ & 45.7 & $\begin{array}{l}\text { gray-brown clayey silt } w / \text { pebbles, } \\
\text { large stones, mottled to } 9.10 \mathrm{~m} \text {; then } \\
\text { brown clayey silt }\end{array}$ \\
\hline 2 & 79573 & $9.30-9.75$ & $10-15-17$ & 45.7 & brown clayey silt w/pebbles, stones \\
\hline 2 & $79 S 74$ & $9.75-10.21$ & $9-14-22$ & 45.7 & $\begin{array}{l}\text { brown clayey silt w/pebbles, few } \\
\text { stones, moist; gray pebbly/sandy zone } \\
10.2 \mathrm{~cm} \text { thick at } 10.06 \mathrm{~m}\end{array}$ \\
\hline 2 & 79575 & $10.21-10.67$ & $15-12-17$ & 15.2 & gray-brown clayey silt $\mathrm{w} /$ large stones \\
\hline 2 & $79 S 76$ & $10.67-11.13$ & $10-11-14$ & 45.7 & $\begin{array}{l}\text { water-bearing dolomite gravel and } \\
\text { stones at } 10.67 \text { to } 10.83 \mathrm{~m} \text {; contact } \\
\text { w/brown clayey silt } \mathrm{w} / \text { pebbles, stones, } \\
\text { moist }\end{array}$ \\
\hline
\end{tabular}

\begin{tabular}{lllllll}
\hline 2 & 79577 & $11.13-11.58$ & $7-12-16$ & 35.6 & $\begin{array}{l}\text { brown-gray clayey silt w/pebbles, } \\
\text { stones, hard, moist }\end{array}$ \\
\hline 2 & 79578 & $11.58-12.04$ & $12-19-37$ & 45.7 & $\begin{array}{l}\text { brown-gray clayey silt w/numerous } \\
\text { pebbles, stones; } 15.2 \text { cm gray gravel- } \\
\text { ly silt at } 11.89 \mathrm{~m}\end{array}$ \\
\hline 2 & 79579 & $12.04-12.50$ & $24-24-22$ & 45.7 & $\begin{array}{l}\text { gravel to } 12.19 \mathrm{~m} \text { gray clayey silt } \\
\text { coarsening to gravelly clay at } 12.34 \\
\text { mi contact w/clayey silt }\end{array}$ \\
\hline 2 & 79580 & $12.50-12.95$ & $17-20-23$ & 40.6 & $\begin{array}{l}\text { brown-gray clayey silt w/few pebbles, } \\
\text { minor oxidation, moist }\end{array}$ \\
\hline 2 & 79581 & $12.95-13.41$ & $42-22-20$ & 35.6 & $\begin{array}{l}\text { gray clayey silt w/pebbles to } 13.11 \mathrm{~m} ; \\
\text { contact w/brown clayey silt w/pebbles, } \\
\text { gritty, moist; soft }\end{array}$ \\
\hline 2 & 79582 & $13.41-13.87$ & $10-15-17$ & 35.6 & $\begin{array}{l}\text { gray-brown clayey silt w/pebbles, } \\
\text { stones }\end{array}$ \\
\hline
\end{tabular}


Table 2.--Test-well construction data and lithologic description of cores at Plot M--Continued

Test well 29--Continued

\begin{tabular}{|c|c|c|c|c|c|}
\hline $\begin{array}{l}\text { Unit } \\
\text { No. }\end{array}$ & $\begin{array}{l}\text { Sample } \\
\text { No. }\end{array}$ & $\begin{array}{c}\text { Depth } \\
\text { interval } \\
\text { (meters) }\end{array}$ & $\begin{array}{l}\text { Blow } \\
\text { counts }\end{array}$ & $\begin{array}{l}\text { Recovery } \\
\quad(\mathrm{cm})\end{array}$ & Description \\
\hline 3 & 79583 & $13.87-14.35$ & $19-17-21$ & 40.6 & $\begin{array}{l}\text { gray-brown clayey silt w/pebbles to } \\
14.02 \mathrm{~m} \text {; then gray silt w/pebbles, } \\
\text { stones }\end{array}$ \\
\hline 3 & 79584 & $14.35-14.78$ & & 35.6 & $\begin{array}{l}\text { brown-gray clayey silt w/pebbles, } \\
\text { stones }\end{array}$ \\
\hline 3 & 79585 & $14.78-15.24$ & $12-17-20$ & 45.7 & $\begin{array}{l}\text { brown-gray clayey silt w/pebbles, } \\
\text { mottled, moist }\end{array}$ \\
\hline 3 & 79586 & $15.24-15.70$ & $23-35-26$ & 45.7 & $\begin{array}{l}\text { gray-brown silt w/pebbles, stones, } \\
\text { moist, hard }\end{array}$ \\
\hline 3 & 79587 & $15.70-16.15$ & $8-9-12$ & 45.7 & $\begin{array}{l}\text { brown silt w/few pebbles, stones, } \\
\text { moist, soft }\end{array}$ \\
\hline 3 & $79 S 88$ & $16.15-16.61$ & $8-13-20$ & 50.8 & $\begin{array}{l}\text { brown silt } w / \text { few pebbles, stones, } \\
\text { moist, soft }\end{array}$ \\
\hline 3 & $79 S 89$ & $16.61-17.07$ & $16-24-24$ & 61.0 & $\begin{array}{l}\text { brown silt, water-bearing, soft to } \\
16.92 \mathrm{~m} \text {; contact w/gray clayey silt; } \\
\text { contact clayey silt w/pebbles }\end{array}$ \\
\hline 3 & 79590 & $17.07-17.52$ & $11-25-26$ & 35.6 & $\begin{array}{l}\text { gray clayey silt w/few pebbles, very } \\
\text { hard, dry }\end{array}$ \\
\hline 3 & 79591 & $17.52-17.98$ & $20-34-29$ & 35.6 & $\begin{array}{l}\text { gray-brown clayey silt } w / f e w \text { pebbles, } \\
\text { dense, dry to moist }\end{array}$ \\
\hline 3 & 79592 & $17.98-18.44$ & $24-32-25$ & 61.0 & $\begin{array}{l}\text { gray-brown clayey silt w/few pebbles, } \\
\text { dense, dry, hard }\end{array}$ \\
\hline 3 & 79593 & $18.44-18.90$ & $16-23-23$ & 50.8 & $\begin{array}{l}\text { brown-gray clayey silt w/pebbles, } \\
\text { moist, hard }\end{array}$ \\
\hline 3 & 79594 & $18.90-19.35$ & $16-18-32$ & 61.0 & $\begin{array}{l}\text { brown-gray clayey silt w/pebbles, } \\
\text { moist }\end{array}$ \\
\hline
\end{tabular}


Table 2.--Test-well construction data and lithologic description of cores at Plot M--Continued

Test well 29--Continued

\begin{tabular}{|c|c|c|c|c|c|}
\hline $\begin{array}{l}\text { Unit } \\
\text { No. }\end{array}$ & $\begin{array}{l}\text { Sample } \\
\text { No. }\end{array}$ & $\begin{array}{c}\text { Depth } \\
\text { interval } \\
\text { (meters) } \\
\end{array}$ & $\begin{array}{l}\text { Blow } \\
\text { counts }\end{array}$ & $\begin{array}{l}\text { Recovery } \\
\quad(\mathrm{cm})\end{array}$ & Description \\
\hline 3 & 79595 & $19.35-19.81$ & $21-12-20$ & 35.6 & $\begin{array}{l}\text { gray clayey silt to } 19.51 \mathrm{~m} \text {; sand } \\
\text { partings at } 19.51 \mathrm{~m} \text {; contact w/thin } \\
\text { brown silt w/pebbles, stones, moist } \\
\text { to wet, gritty }\end{array}$ \\
\hline 3 & 79596 & $19.81-20.27$ & $23-17-22$ & 61.0 & $\begin{array}{l}\text { gray clayey silt to } 19.96 \mathrm{~m} \text {; contact } \\
\text { w/brown silt } \mathrm{w} / \text { pebbles, stones, moist }\end{array}$ \\
\hline 3 & 79597 & $20.27-20.73$ & $15-19-20$ & 40.6 & $\begin{array}{l}\text { brown wet silt to } 20.42 \mathrm{~m} \text {; contact } \mathrm{w} / \\
\text { gray clayey silt } \mathrm{w} / \text { pebbles, stones }\end{array}$ \\
\hline 4 & 79598 & $20.73-21.18$ & $14-21-27$ & 45.7 & $\begin{array}{l}\text { brown-gray clayey silt w/pebbles, } \\
\text { stones, moist, dense, hard }\end{array}$ \\
\hline 4 & 79599 & $21.18-21.64$ & $31-36-26$ & 50.8 & $\begin{array}{l}\text { gray clayey silt w/pebbles, stones, } \\
\text { moist, dense, hard }\end{array}$ \\
\hline 4 & 795100 & $21.64-22.10$ & $26-33-35$ & 50.8 & $\begin{array}{l}\text { gray clayey silt w/pebbles; brown } \\
\text { silty seam at } 21.79 \mathrm{~m}\end{array}$ \\
\hline 4 & 795101 & $22.10-22.56$ & $24-38-30$ & 35.6 & $\begin{array}{l}\text { gray clayey silt w/few pebbles, dry } \\
\text { to moist, dense, very hard }\end{array}$ \\
\hline 4 & 795102 & $22.56-23.01$ & $40-50 / 5$ & 25.4 & $\begin{array}{l}\text { gray clayey silt w/few pebbles, dry } \\
\text { to moist, dense, very hard }\end{array}$ \\
\hline 4 & 795103 & $23.01-23.47$ & $38-45-31$ & 35.6 & $\begin{array}{l}\text { gray-brown silt w/few pebbles, dense, } \\
\text { very hard }\end{array}$ \\
\hline 4 & 795104 & $23.47-23.93$ & $33-50 / 5$ & 25.4 & $\begin{array}{l}\text { gray-brown clayey silt w/few pebbles, } \\
\text { dry to moist, hard }\end{array}$ \\
\hline 4 & $79 s 105$ & $23.93-24.38$ & $38-52-52$ & 35.6 & $\begin{array}{l}\text { gray-brown clayey silt w/few pebbles, } \\
\text { dry to moist, hard }\end{array}$ \\
\hline
\end{tabular}

EOB $25.91 \mathrm{~m}$ 
Table 2.--Test-well construction data and lithologic description of cores at Plot $\mathrm{M}$--Continued

\section{Test well 30}

\section{Completion date: October 3, 1979}

Location: 65 meters north-northeast of the northeast corner of Plot M, 15 meters east of test well 5

Site and well construction data: 2 test wells; $18.44 \mathrm{~m}, 24.54 \mathrm{~m}$

Altitude of land surface: $18.44 \mathrm{~m}$ test well. . . . . . $203.79 \mathrm{~m}$

$24.54 \mathrm{~m}$ test well. . . . . . . $203.76 \mathrm{~m}$

Altitude of measuring point (top of casing): $18.44 \mathrm{~m}$ test well. . $204.10 \mathrm{~m}$

$24.54 \mathrm{~m}$ test well. $204.08 \mathrm{~m}$

Depth of bottom of piezometer from land surface: $18.44 \mathrm{~m}$ test well. $.18 .13 \mathrm{~m}$

$24.54 \mathrm{~m}$ test well. $24.22 \mathrm{~m}$

Type of piezometer: $1.22 \mathrm{~m}$ slotted PVC pipe, $5.08 \mathrm{~cm}$ diameter

\begin{tabular}{|c|c|c|c|c|c|}
\hline $\begin{array}{l}\text { Unit } \\
\text { No. }\end{array}$ & $\begin{array}{l}\text { Sample } \\
\text { No. }\end{array}$ & $\begin{array}{c}\text { Depth } \\
\text { interval } \\
\text { (meters) }\end{array}$ & $\begin{array}{l}\text { Blow } \\
\text { counts }\end{array}$ & $\begin{array}{l}\text { Recovery } \\
\quad(\mathrm{cm})\end{array}$ & Description \\
\hline 1 & $79 S 159$ & $0-0.46$ & $3-6-6$ & 15.2 & brown clayey soil w/stones \\
\hline 1 & $79 S 160$ & $0.46-0.91$ & $10-12-12$ & 30.5 & $\begin{array}{l}\text { brown clayey soil w/stones, dry, root } \\
\text { tubes }\end{array}$ \\
\hline 1 & $79 S 161$ & $0.91-1.37$ & $11-16-16$ & 35.6 & $\begin{array}{l}\text { yellow-brown silty clay w/stones, } \\
\text { plant fragments, dry }\end{array}$ \\
\hline 1 & $79 S 162$ & $1.37-1.83$ & $16-25-29$ & 25.4 & $\begin{array}{l}\text { brown silty clay, plant fragments, } \\
\text { hard, dense, dry }\end{array}$ \\
\hline 1 & $79 S 163$ & $1.83-2.29$ & $8-10-16$ & 40.6 & $\begin{array}{l}\text { yellow-brown silty clay w/pebbles; } \\
\text { grading to brown-gray silty clay } \\
\text { w/stones }\end{array}$ \\
\hline 2 & $79 S 164$ & $2.29-2.74$ & & NR & \\
\hline 2 & $79 S 165$ & $2.74-3.20$ & $19-18-14$ & NR & \\
\hline 2 & $79 S 166$ & $3.20-3.66$ & $10.15-16$ & 10.2 & $\begin{array}{l}\text { brown-gray silty clay w/pebbles and } \\
\text { stones, dry }\end{array}$ \\
\hline 2 & $79 S 167$ & $3.66-4.12$ & $30-22-16$ & 15.2 & $\begin{array}{l}\text { brown-gray silty clay } w / \text { pebbles and } \\
\text { stones, dry }\end{array}$ \\
\hline
\end{tabular}


Table 2.--Test-well construction data and lithologic description of cores at Plot M--Continued

Test well $30--$ Continued

\begin{tabular}{|c|c|c|c|c|c|}
\hline $\begin{array}{l}\text { Unit } \\
\text { No. }\end{array}$ & $\begin{array}{l}\text { Sample } \\
\text { No. }\end{array}$ & $\begin{array}{c}\text { Depth } \\
\text { interval } \\
\text { (meters) }\end{array}$ & $\begin{array}{l}\text { Blow } \\
\text { counts }\end{array}$ & $\begin{array}{l}\text { Recovery } \\
\quad(\mathrm{cm})\end{array}$ & Description \\
\hline 2 & $79 S 168$ & $4.12-4.57$ & $9-12-13$ & 35.6 & $\begin{array}{l}\text { brown-gray silty clay, silt w/stones, } \\
\text { dry, brittle }\end{array}$ \\
\hline 2 & $79 S 169$ & $4.57-5.03$ & $35-19-18$ & 15.2 & $\begin{array}{l}\text { brown-gray silty clay w/pebbles, } \\
\text { stones, rocks }\end{array}$ \\
\hline 2 & $79 S 170$ & $5.03-5.49$ & $13-14-14$ & 15.2 & $\begin{array}{l}\text { brown-gray silty clay w/stones, hard, } \\
\text { dry }\end{array}$ \\
\hline 2 & 795171 & $5.49-5.94$ & $9-12-21$ & 35.6 & $\begin{array}{l}\text { brown-gray silty clay to } 5.55 \mathrm{~m} ; 5.1 \\
\mathrm{~cm} \text { wet gravelly sand at } 5.55 \mathrm{~m}, 5.79 \\
\mathrm{~m}, 5.94 \mathrm{~m} \text {; contact w/brown-gray silty } \\
\text { clay w/pebbles, moist, soft }\end{array}$ \\
\hline 2 & $79 S 172$ & $5.94-6.40$ & $12-15-17$ & 15.2 & $\begin{array}{l}\text { brown-gray clayey silt w/pebbles, } \\
\text { stones, gravel, sandy }\end{array}$ \\
\hline 2 & 795173 & $6.40-6.96$ & $18-18-13$ & 10.2 & brown-gray clayey silt $\mathrm{w} /$ pebbles, wet \\
\hline 2 & $79 S 174$ & $6.96-7.32$ & $7-8-12$ & 15.2 & $\begin{array}{l}\text { gray-brown clayey silt w/pebbles, } \\
\text { moist }\end{array}$ \\
\hline 2 & $79 S 175$ & $7.32-7.77$ & $7-10-12$ & 50.8 & $\begin{array}{l}\text { brown clayey silt w/pebbles, moist, } \\
\text { soft to } 7.62 \mathrm{~m} \text {; grading to brown-gray } \\
\text { clayey silt w/pebbles, stones, dry to } \\
\text { moist, dense, hard }\end{array}$ \\
\hline 2 & $79 S 176$ & $7.77-8.23$ & $10-15-16$ & 50.8 & $\begin{array}{l}\text { gray clayey silt w/pebbles, dry, hard } \\
\text { to } 8.10 \mathrm{~m} \text {; grading to gray-brown } \\
\text { clayey silt w/pebbles, moist, soft }\end{array}$ \\
\hline 2 & 795177 & $8.23-8.69$ & $15-16-11$ & 35.6 & $\begin{array}{l}\text { gray-brown clayey silt w/pebbles, } \\
\text { stones }\end{array}$ \\
\hline 2 & $79 S 178$ & $8.69-9.14$ & $13-17-21$ & 45.7 & $\begin{array}{l}\text { gray clayey silt w/few pebbles, hard, } \\
\text { dense, dry }\end{array}$ \\
\hline 2 & 795179 & $9.14-9.60$ & $18-20-18$ & 30.5 & $\begin{array}{l}\text { gray clayey silt w/few pebbles, hard, } \\
\text { dense, dry }\end{array}$ \\
\hline
\end{tabular}


Table 2.--Test-well construction data and lithologic description of cores at Plot M--Continued

Test well 30--Continued

\begin{tabular}{|c|c|c|c|c|c|}
\hline $\begin{array}{l}\text { Unit } \\
\text { No. }\end{array}$ & $\begin{array}{l}\text { Sample } \\
\text { No. }\end{array}$ & $\begin{array}{c}\text { Depth } \\
\text { interval } \\
\text { (meters) }\end{array}$ & $\begin{array}{l}\text { Blow } \\
\text { counts }\end{array}$ & $\begin{array}{l}\text { Recovery } \\
\quad(\mathrm{cm})\end{array}$ & Description \\
\hline 2 & $79 S 180$ & $9.60-10.06$ & $21-34-33$ & 30.5 & $\begin{array}{l}\text { gray clayey silt w/few pebbles, hard, } \\
\text { dense, dry }\end{array}$ \\
\hline 2 & 795181 & $10.06-10.52$ & $36-35-39$ & NR & rocks \\
\hline 3 & $79 S 182$ & $10.52-10.97$ & & NR & rocks \\
\hline 3 & $79 S 183$ & $10.97-11.43$ & & NR & rocks \\
\hline 3 & $79 S 184$ & $11.43-11.89$ & $15-33-20$ & 40.6 & $\begin{array}{l}\text { brown clayey silt w/numerous stones, } \\
\text { gritty, hard, dry, brittle }\end{array}$ \\
\hline 3 & $79 S 185$ & $11.89-12.34$ & & NR & rocks \\
\hline 3 & $79 S 186$ & $12.34-13.11$ & $24-19-10$ & 20.3 & $\begin{array}{l}\text { brown clayey silt w/stones, gritty, } \\
\text { moist to wet, soft }\end{array}$ \\
\hline 3 & $79 S 187$ & $13.11-13.56$ & $7-9-18$ & 35.6 & $\begin{array}{l}\text { brown clayey silt } w / \text { pebbles, stones, } \\
\text { gritty, moist, soft; grading to brown- } \\
\text { gray clayey silt } w / \text { few pebbles }\end{array}$ \\
\hline 3 & $79 S 188$ & $13.56-14.02$ & $10-18-27$ & 45.7 & $\begin{array}{l}\text { brown-gray clayey silt w/few pebbles; } \\
\text { grading to gray clayey silt }\end{array}$ \\
\hline 3 & 795189 & $14.02-14.48$ & $22-31-31$ & 30.5 & $\begin{array}{l}\text { gray clayey silt w/few pebbles, dry, } \\
\text { hard, brittle }\end{array}$ \\
\hline 3 & 795190 & $14.48-14.94$ & $18-24-26$ & 61.0 & $\begin{array}{l}\text { gray clayey silt w/few pebbles, dry, } \\
\text { hard, brittle }\end{array}$ \\
\hline 3 & 795191 & $14.94-15.39$ & $21-21-27$ & 50.8 & $\begin{array}{l}\text { gray clayey silt w/few pebbles, dry } \\
\text { to moist, hard }\end{array}$ \\
\hline 3 & $79 S 192$ & $15.39-15.85$ & $13-20-27$ & 61.0 & $\begin{array}{l}\text { gray clayey silt w/few pebbles, dry } \\
\text { to moist, hard; sand parting at } 15.70 \mathrm{~m}\end{array}$ \\
\hline 3 & 795193 & $15.85-16.31$ & $18-21-19$ & 35.6 & $\begin{array}{l}\text { gray clayey silt w/few pebbles, dry } \\
\text { to moist, hard, brittle }\end{array}$ \\
\hline
\end{tabular}


Table 2.--Test-well construction data and lithologic description of cores at Plot M--Continued

\section{Test well 30--Continued}

\begin{tabular}{|c|c|c|c|c|c|}
\hline $\begin{array}{l}\text { Unit } \\
\text { No. }\end{array}$ & $\begin{array}{l}\text { Sample } \\
\text { No. }\end{array}$ & $\begin{array}{c}\text { Depth } \\
\text { interval } \\
\text { (meters) }\end{array}$ & $\begin{array}{l}\text { Blow } \\
\text { counts }\end{array}$ & $\begin{array}{l}\text { Recovery } \\
\quad(\mathrm{cm})\end{array}$ & Description \\
\hline 3 & 795194 & $16.61-16.76$ & $8-50 / 5$ & 35.6 & $\begin{array}{l}\text { gray clayey silt } \mathrm{w} / \text { few pebbles, dry } \\
\text { to moist, hard, brittle (stones at } \\
\text { bottom) to } 16.61 \mathrm{~m}\end{array}$ \\
\hline 3 & $79 S 195$ & $16.76-17.22$ & $25-21-20$ & 40.6 & $\begin{array}{l}\text { gray clayey silt; grading to brown } \\
\text { clayey silt w/pebbles, stones, dry }\end{array}$ \\
\hline 3 & 795196 & $17.22-17.68$ & $24-20-22$ & 30.5 & $\begin{array}{l}\text { brown clayey silt w/pebbles, stones, } \\
\text { gritty, moist }\end{array}$ \\
\hline 4 & $79 S 197$ & $17.68-18.14$ & $17-18-18$ & 35.6 & $\begin{array}{l}\text { brown clayey silt w/pebbles, gritty, } \\
\text { soft, moist; grading to gray-brown } \\
\text { clayey silt }\end{array}$ \\
\hline 4 & $79 S 198$ & $18.14-18.59$ & & NR & \\
\hline 4 & 795199 & $18.59-19.05$ & & NR & rocks \\
\hline 4 & 795200 & $19.05-19.51$ & $25-26-26$ & 35.6 & $\begin{array}{l}\text { gray-brown clayey silt w/few pebbles, } \\
\text { moist, hard }\end{array}$ \\
\hline 4 & 795201 & $19.51-19.96$ & $34-50 / 4$ & 15.2 & $\begin{array}{l}\text { gray-brown clayey silt } \mathrm{w} / \mathrm{stones,} \text { dry } \\
\text { to moist, brittle }\end{array}$ \\
\hline 4 & 795202 & $19.96-20.42$ & $19-30-38$ & 50.8 & $\begin{array}{l}\text { gray silt w/stones, dry, brittle, } \\
\text { soft, flaky }\end{array}$ \\
\hline 4 & 795203 & $20.42-20.88$ & $41-35-34$ & 40.6 & $\begin{array}{l}\text { gray silt w/few stones, dry, brittle, } \\
\text { soft, flaky }\end{array}$ \\
\hline 4 & 795204 & $20.88-21.33$ & $28-37-31$ & 35.6 & gray silt, dry, flaky \\
\hline 4 & 795205 & $21.33-21.79$ & $15-23-25$ & 45.7 & gray silt, dry, flaky \\
\hline 4 & 795206 & $21.79-22.25$ & $20-35-28$ & 30.5 & gray silt, dry, flaky \\
\hline 4 & 795207 & $22.25-22.71$ & $19-36-38$ & 50.8 & gray silt, dry, flaky \\
\hline
\end{tabular}


Table 2.--Test-well construction data and lithologic description of cores at Plot M--Continued

Test well 30--Continued

\begin{tabular}{|c|c|c|c|c|c|}
\hline $\begin{array}{l}\text { Unit } \\
\text { No. }\end{array}$ & $\begin{array}{c}\text { Sample } \\
\text { No. }\end{array}$ & $\begin{array}{c}\text { Depth } \\
\text { interval } \\
\text { (meters) }\end{array}$ & $\begin{array}{l}\text { Blow } \\
\text { counts }\end{array}$ & $\begin{array}{l}\text { Recovery } \\
\quad(\mathrm{cm})\end{array}$ & Description \\
\hline 4 & 795208 & $22.71-23.16$ & $35-32-30$ & 40.6 & $\begin{array}{l}\text { gray silt, dry, flaky; } 0.63 \mathrm{~cm} \text { wide } \\
45^{\circ} \text { fracture fitted w/clean dry sand }\end{array}$ \\
\hline 4 & 795209 & $23.16-23.62$ & $20-27-36$ & 61.0 & $\begin{array}{l}\text { gray silt, dry, } 0.63 \mathrm{~cm} \text { wide } 45^{\circ} \\
\text { fracture filled w/clean dry sand; sano } \\
\text { parting at } 23.17 \mathrm{~m} \text {; } 5.1 \mathrm{~cm} \text { dry layer } \\
\text { at } 23.20 \mathrm{~m} \text {; flaky to } 23.47 \mathrm{~m} \text {; contact } \\
\text { w/wet sandy, light brown silt, soft, } \\
\text { dense }\end{array}$ \\
\hline 4 & 795210 & $23.62-24.08$ & $29-42-50 / 4$ & 30.5 & gray silt, dry \\
\hline 4 & 795211 & $24.08-24.53$ & $23-34-56$ & 45.7 & $\begin{array}{l}\text { wet sandy silt to } 24.08 \mathrm{~m} \text {; contact } \\
\text { w/gray silt, moist, hard }\end{array}$ \\
\hline
\end{tabular}

EOB $24.99 \mathrm{~m}$ 
Table 2.--Test-well construction data and lithologic description of cores at Plot M--Continued

\section{Test well 33}

Completion date: June 11, 1980

Location: 2 meters south of the south boundary of Plot M, halfway between the southeast corner and the southwest corner of the burial site

Site and well construction data:

Altitude of land surface ............ $215.20 \mathrm{~m}$

Altitude of measuring point (top of casing). . . . $215.50 \mathrm{~m}$

Depth to bottom of piezometer from land surface. . . $27.10 \mathrm{~m}$

Type of piezometer: $1.22 \mathrm{~m}$ slotted PVC pipe, $5.08 \mathrm{~cm}$ diameter

\begin{tabular}{cccccc}
\hline $\begin{array}{c}\text { Unit } \\
\text { No. }\end{array}$ & $\begin{array}{c}\text { Sample } \\
\text { No. }\end{array}$ & $\begin{array}{c}\text { Depth } \\
\text { interval } \\
\text { (meters) }\end{array}$ & $\begin{array}{c}\text { Blow } \\
\text { counts }\end{array}$ & $\begin{array}{c}\text { Recovery } \\
\text { (cm) }\end{array}$ & Description \\
\hline 1 & 80585 & $0-0.46$ & $1-4-5$ & 25.4 & topsoil $(10 \mathrm{~cm})$, yellow-brown clay soil \\
\hline 1 & 80586 & $0.46-0.91$ & $3-4-4$ & 20.3 & $\begin{array}{l}\text { brown clayey soil w/pebbles, stones, } \\
\text { dry; plant fragments; wide variety of } \\
\text { rocks, minerals }\end{array}$ \\
\hline
\end{tabular}

\begin{tabular}{ccccccc}
\hline 1 & 80587 & $0.91-1.37$ & $3-4-4$ & 10.2 & $\begin{array}{l}\text { brown silty clay w/pebbles, stones, } \\
\text { moist, oxidized, soft }\end{array}$ \\
\hline 1 & 80588 & $1.37-1.83$ & $5-7-7$ & 25.4 & $\begin{array}{l}\text { brown silty clay w/pebbles, stones, } \\
\text { moist, oxidized, soft, plant fragments }\end{array}$ \\
\hline 1 & 80589 & $1.83-2.29$ & $5-10-18$ & 30.5 & $\begin{array}{l}\text { brown silty clay w/pebbles, stones, } \\
\text { moist, oxidized, soft; grading down- } \\
\text { wards to brown silty clay, dry, hard }\end{array}$ \\
\hline 1 & 80590 & $2.29-2.74$ & $14-21-26$ & 40.6 & $\begin{array}{l}\text { brown silty clay w/pebbles, stones, } \\
\text { dry, hard, wide variety of rocks } \\
\text { (dolomite, shale, granite), minerals } \\
\text { (including pyrite); oxidized, mottled }\end{array}$ \\
\hline 1 & 80591 & $2.74-3.20$ & $15-16-17$ & 45.7 & $\begin{array}{l}\text { brown silty clay w/pebbles, dry, hard; } \\
\text { moist at 3.20 m }\end{array}$ \\
\hline 1 & 80592 & $3.20-3.66$ & $8-10-12$ & 40.6 & $\begin{array}{l}\text { brown silty clay w/pebbles to } 3.30 \mathrm{~m} ; \\
\text { clay w/silt and sand, moist to wet, } \\
\text { soft to 3.40 m; brown silty clay w/ } \\
\text { pebbles, dry, hard }\end{array}$ \\
\hline
\end{tabular}


Table 2.--Test-well construction data and lithologic description of cores at Plot M--Continued

Test well 33--Continued

\begin{tabular}{cccccl}
\hline $\begin{array}{c}\text { Unit } \\
\text { No. }\end{array}$ & $\begin{array}{c}\text { Sample } \\
\text { No. }\end{array}$ & $\begin{array}{c}\text { Depth } \\
\text { interval } \\
\text { (meters) }\end{array}$ & $\begin{array}{c}\text { Blow } \\
\text { counts }\end{array}$ & $\begin{array}{c}\text { Recovery } \\
\text { (cm) }\end{array}$ & \multicolumn{1}{c}{ Description } \\
\hline 1 & 80593 & $3.66-4.12$ & $9-12-16$ & 35.6 & $\begin{array}{l}\text { brown silty clay w/pebbles, dry, hard, } \\
\text { wide variety of rocks (numerous black } \\
\text { shale grains) and minerals }\end{array}$ \\
\hline 1 & 80594 & $4.12-4.57$ & $10-12-14$ & 50.8 & $\begin{array}{l}\text { brown silty clay w/pebbles, few } \\
\text { stones, dry, hard, numerous dolomite } \\
\text { and black shale grains }\end{array}$ \\
\hline 1 & 80595 & $4.57-5.03$ & $6-10-13$ & 25.4 & $\begin{array}{l}\text { brown clayey silt w/pebbles, dry, } \\
\text { hard, numerous black shale grains }\end{array}$ \\
\hline 1 & 80596 & $5.03-5.49$ & $16-18-21$ & 50.8 & $\begin{array}{l}\text { brown clayey silt w/pebbles, few } \\
\text { stones to 5.28 m; contact w/gray- } \\
\text { brown clayey silt w/pebbles, dry to } \\
\text { moist, hard, mostly dolomite and } \\
\text { black shale pebbles }\end{array}$ \\
\hline
\end{tabular}

\begin{tabular}{|c|c|c|c|c|c|}
\hline 2 & 80597 & $5.49-5.94$ & & NR & \\
\hline 2 & 80598 & $5.94-6.40$ & & $N R$ & \\
\hline 2 & 80599 & $6.40-6.86$ & $9-11-17$ & 25.4 & $\begin{array}{l}\text { gray-brown clayey silt } w / \text { pebbles, } \\
\text { stones, dry to moist, soft, wide } \\
\text { variety of rocks and minerals }\end{array}$ \\
\hline 2 & 805100 & $6.86-7.32$ & $19-22-20$ & 25.4 & $\begin{array}{l}\text { gray-brown clayey silt w/pebbles, } \\
\text { stones; grading downwards to yellow- } \\
\text { brown clayey silt, oxidized, mottled, } \\
\text { numerous dolomite and black shale } \\
\text { grains, vertical fracture }\end{array}$ \\
\hline 2 & $80 S 101$ & $7.32-7.77$ & $7-10-16$ & 40.6 & $\begin{array}{l}\text { gray-brown clayey silt w/pebbles, } \\
\text { stones, dry to moist, mostly dolomite } \\
\text { and black shale grains }\end{array}$ \\
\hline 2 & 805102 & $7.77-8.23$ & $9-12-14$ & 61.0 & $\begin{array}{l}\text { gray-brown clayey silt w/pebbles, stones } \\
\text { to } 8.33 \mathrm{~m} \text {; contact } \mathrm{w} / \mathrm{brown} \text { sandy silt, } \\
\text { moist to wet, soft; } 25 \mathrm{~cm} \text { inclined } \\
\text { fracture, oxidized, } \mathrm{w} / 2.5 \mathrm{~cm} \text { bleach } \\
\text { zone }\end{array}$ \\
\hline
\end{tabular}


Table 2.--Test-well construction data and lithologic description of cores at Plot M--Continued

Test well 33--Continued

\begin{tabular}{cccccl}
\hline $\begin{array}{c}\text { Unit } \\
\text { No. }\end{array}$ & $\begin{array}{c}\text { Sample } \\
\text { No. }\end{array}$ & $\begin{array}{c}\text { Depth } \\
\text { interval } \\
\text { (meters) }\end{array}$ & $\begin{array}{c}\text { Blow } \\
\text { counts }\end{array}$ & $\begin{array}{c}\text { Recovery } \\
(\mathrm{cm})\end{array}$ & Description \\
\hline 2 & 80 S103 & $8.23-8.69$ & $10-14-18$ & 20.3 & $\begin{array}{l}\text { brown sandy silt w/pebbles, stones; } \\
\text { grading downwards to gray-brown } \\
\text { clayey silt w/pebbles, stones, moist, } \\
\text { soft, gritty }\end{array}$ \\
\hline 2 & 805104 & $8.69-9.14$ & $11-14-17$ & 30.5 & $\begin{array}{l}\text { gray-brown clayey silt w/pebbles, } \\
\text { stones, moist, soft, gritty }\end{array}$ \\
\hline 2 & 80 s105 & $9.14-9.60$ & $12-15-16$ & 20.3 & $\begin{array}{l}\text { gray-brown clayey silt w/pebbles, few } \\
\text { stones, moist, soft, mottled, mostly } \\
\text { dolomite and black shale grains }\end{array}$ \\
\hline 2 & 80 s106 & $9.60-10.06$ & $13-14-17$ & 61.0 & $\begin{array}{l}\text { gray-brown clayey silt w/pebbles to } \\
\text { 9.75 m; contact w/gray-brown clayey } \\
\text { silt w/numerous pebbles, stones, } \\
\text { rocks; inclined fracture w/2 cm bleach } \\
\text { zone }\end{array}$ \\
\hline
\end{tabular}

\begin{tabular}{|c|c|c|c|c|c|}
\hline 2 & 805107 & $10.06-10.52$ & $9-12-15$ & 45.7 & $\begin{array}{l}\text { gray-brown clayey silt w/pebbles, } \\
\text { stones, moist to wet, soft, mostly } \\
\text { dolomite grains }\end{array}$ \\
\hline 2 & $80 S 108$ & $10.52-10.97$ & $24-28-27$ & 35.6 & $\begin{array}{l}\text { gray-brown clayey silt w/pebbles, } \\
\text { stones, rocks; horizontal fracture } \\
\text { w/bleach zone at } 10.61 \mathrm{~m}\end{array}$ \\
\hline 2 & 805109 & $10.97-11.43$ & $9-21-15$ & 30.5 & $\begin{array}{l}\text { gray-brown clayey silt w/pebbles, } \\
\text { stones, rocks }\end{array}$ \\
\hline 2 & $80 S 110$ & $11.43-11.89$ & $7-11-13$ & 40.6 & $\begin{array}{l}\text { gray-brown clayey silt w/pebbles, } \\
\text { stones, rocks to } 11.68 \mathrm{~m} \text {; contact } \\
\text { w/brown silty clay, sandy, gritty, } \\
\text { moist, soft }\end{array}$ \\
\hline 2 & 805111 & $11.89-12.34$ & $9-13-16$ & 35.6 & $\begin{array}{l}\text { brown silty clay, sandy, moist, soft } \\
\text { to } 11.98 \mathrm{~m} \text {; contact } \mathrm{w} / \text { brown clayey } \\
\text { silt } \mathrm{w} / \text { pebbles; grading downwards to } \\
\text { pink clayey silt }\end{array}$ \\
\hline
\end{tabular}


Table 2.--Test-well construction data and lithologic description of cores at Plot M--Continued

Test well 33--Continued

\begin{tabular}{cccccc}
\hline $\begin{array}{l}\text { Unit } \\
\text { No. }\end{array}$ & $\begin{array}{c}\text { Sample } \\
\text { No. }\end{array}$ & $\begin{array}{c}\text { Depth } \\
\text { interval } \\
\text { (meters) }\end{array}$ & $\begin{array}{c}\text { Blow } \\
\text { counts }\end{array}$ & $\begin{array}{c}\text { Recovery } \\
(\mathrm{cm})\end{array}$ & Description \\
\hline 2 & $80 \mathrm{~s} 112$ & $12.34-12.80$ & $10-11-15$ & 35.6 & $\begin{array}{l}\text { pink clayey silt w/rocks (all dolo- } \\
\text { mite) i grading downwards to brown silt, } \\
\text { dry to moist, soft; grading downwards } \\
\text { to brown silt w/sand, wet, soft }\end{array}$ \\
\hline
\end{tabular}

$2805113 \quad 12.80-13.26 \quad 14-22-25 \quad 35.6 \quad$ brown silt $\mathrm{w} / \mathrm{s}$ and to $12.85 \mathrm{~m}$; contact
w/gray-brown clayey silt w/pebbles, $1.3 \mathrm{~cm}$ sand layer at $13.05 \mathrm{~m}, 5 \mathrm{~cm}$ pink clay layer at $13.10 \mathrm{~m}$, then graybrown clayey silt, dry, hard

\begin{tabular}{|c|c|c|c|c|c|}
\hline 2 & 805114 & $13.26-13.72$ & $8-12-18$ & 45.7 & $\begin{array}{l}\text { gray-brown clayey silt w/pebbles to } \\
13.51 \mathrm{~m} \text {; contact w/brown silt, moist, } \\
\text { gritty, mostly dolomite grains }\end{array}$ \\
\hline 2 & $80 S 115$ & $13.72-14.17$ & $21-13-18$ & 45.7 & $\begin{array}{l}\text { brown silt w/pebbles, moist, soft, } \\
\text { dolomite, black shale, and pyrite } \\
\text { grains }\end{array}$ \\
\hline 2 & $80 \operatorname{sil} 16$ & $14.17-14.63$ & $12-18-24$ & 61.0 & $\begin{array}{l}\text { brown silt, moist, gritty, soft, } \\
\text { dolomite and black shale grains }\end{array}$ \\
\hline 3 & $80 S 117$ & $14.63-15.09$ & $11-17-19$ & 25.4 & $\begin{array}{l}\text { brown silt, moist, gritty, soft; } \\
\text { grading downwards to gray-brown } \\
\text { clayey silt w/pebbles, stones, dolo- } \\
\text { mite and black shale }\end{array}$ \\
\hline 3 & $80 S 118$ & $15.09-15.55$ & $14-20-29$ & 25.4 & $\begin{array}{l}\text { gray-brown clayey silt w/pebbles, } \\
\text { stones }\end{array}$ \\
\hline 3 & $80 S 119$ & $15.55-16.00$ & & NR & \\
\hline 3 & $80 S 120$ & $16.00-16.46$ & $24-23-24$ & 35.6 & $\begin{array}{l}\text { gray-brown clayey silt w/pebbles, } \\
\text { stones to } 16.05 \mathrm{~m} \text {; contact } \mathrm{w} / \mathrm{wet} \\
\text { sandy dolomite gravel to } 16.15 \mathrm{~m} \text {; } \\
\text { contact w/brown silt, moist, soft }\end{array}$ \\
\hline 3 & $80 S 121$ & $16.46-16.92$ & $8-14-18$ & 35.6 & $\begin{array}{l}\text { brown silt } w / \text { few pebbles, moist, soft, } \\
\text { mostly dolomite grains }\end{array}$ \\
\hline
\end{tabular}


Table 2.--Test-well construction data and lithologic description of cores at Plot M--Continued

Test well 33--Continued

\begin{tabular}{|c|c|c|c|c|c|}
\hline $\begin{array}{l}\text { Unit } \\
\text { No. }\end{array}$ & $\begin{array}{c}\text { Sample } \\
\text { No. }\end{array}$ & $\begin{array}{c}\text { Depth } \\
\text { interval } \\
\text { (meters) }\end{array}$ & $\begin{array}{l}\text { Blow } \\
\text { counts }\end{array}$ & $\begin{array}{l}\text { Recovery } \\
\text { (cm) }\end{array}$ & Description \\
\hline 3 & $80 S 122$ & $16.92-17.37$ & $10-22-37$ & 45.7 & $\begin{array}{l}\text { brown silt } w / \text { few pebbles, moist, soft } \\
2.54 \mathrm{~cm} \text { sand parting at } 17.17 \mathrm{~m} \text { and } \\
\text { at } 17.20 \mathrm{~m}\end{array}$ \\
\hline 3 & $80 S 123$ & $17.37-17.83$ & $36-28-31$ & 30.5 & $\begin{array}{l}\text { light brown silt } \mathrm{w} / \mathrm{sand} \text { to } 17.42 \mathrm{~m} \text {; } \\
\text { contact w/gray-brown clayey silt to } \\
17.48 \mathrm{~m} \text {; contact w/sand to } 17.52 \mathrm{~m} \text {; } \\
\text { contact w/gray-brown clayey silt to } \\
17.63 \mathrm{~m} ; \text { contact } \mathrm{w} / \text { sand }\end{array}$ \\
\hline
\end{tabular}

$3805124 \quad 17.83-18.29 \quad 13-14-21 \quad 15.2$ sand to $17.86 \mathrm{~m}$; contact w/gray-brown clayey silt to $17.96 \mathrm{~m}$; contact $\mathrm{w} /$ sand, gravel to $18.06 \mathrm{~m}$; contact $\mathrm{w} /$ gray-brown silt, no pebbles, stones, dry to moist, hard

\begin{tabular}{|c|c|c|c|c|c|}
\hline 3 & $80 S 125$ & $18.29-18.75$ & $13-14-23$ & 48.3 & gray-brown silt, dry to moist \\
\hline 3 & $80 S 126$ & $18.75-19.20$ & $19-25-25$ & 40.6 & gray-brown silt, dry to moist \\
\hline 3 & $80 S 127$ & $19.20-19.66$ & $19-22-19$ & 40.6 & $\begin{array}{l}\text { gray-brown silt, dry to moist to } \\
19.30 \mathrm{~m} \text {; contact w/brown silt } \mathrm{w} / \mathrm{peb}- \\
\text { bles, stones, dry to moist, soft, } \\
\text { mostly dolomite grains }\end{array}$ \\
\hline 3 & $80 S 128$ & $19.66-20.12$ & $79-38-23$ & 10.2 & $\begin{array}{l}\text { brown silt } w / \text { pebbles, stones, dry to } \\
\text { moist; rock in sampler }\end{array}$ \\
\hline 3 & $80 S 129$ & $20.12-20.57$ & $17-21-21$ & 20.3 & brown silt $w /$ few pebbles, rocks \\
\hline 4 & $80 S 130$ & $20.57-21.03$ & $14-19-18$ & 30.5 & $\begin{array}{l}\text { brown silt } w / \text { few pebbles to } 20.83 \mathrm{~m} \text {; } \\
\text { grading downwards to gray-brown silt } \\
\text { w/few pebbles, stones, dry }\end{array}$ \\
\hline 4 & 805131 & $21.03-21.49$ & $26-32-28$ & 15.2 & $\begin{array}{l}\text { gray-brown silt w/few pebbles, dry, } \\
\text { hard }\end{array}$ \\
\hline 4 & $80 S 132$ & $21.49-21.95$ & $15-29-27$ & 20.3 & $\begin{array}{l}\text { gray-brown silt w/few pebbles, stones, } \\
\text { dry, hard, brittle }\end{array}$ \\
\hline
\end{tabular}


Table 2.--Test-well construction data and lithologic description of cores at Plot M--Continued

\section{Test well 33--Continued}

\begin{tabular}{cccccl}
\hline $\begin{array}{c}\text { Unit } \\
\text { No. }\end{array}$ & $\begin{array}{c}\text { Sample } \\
\text { No. }\end{array}$ & $\begin{array}{c}\text { Depth } \\
\text { interval } \\
\text { (meters) }\end{array}$ & $\begin{array}{c}\text { Blow } \\
\text { counts }\end{array}$ & $\begin{array}{c}\text { Recovery } \\
(\mathrm{cm})\end{array}$ & \multicolumn{1}{c}{ Description } \\
\hline 4 & $80 \mathrm{~s} 133$ & $21.95-22.40$ & $14-27-42$ & 25.4 & $\begin{array}{l}\text { gray-brown silt w/few pebbles, stones, } \\
\text { dry, hard, brittle }\end{array}$ \\
\hline 4 & 805134 & $22.40-22.86$ & $15-21-30$ & 35.6 & $\begin{array}{l}\text { gray-brown silt w/few pebbles, stones, } \\
\text { dry, hard, brittle }\end{array}$ \\
\hline 4 & 805135 & $22.86-23.32$ & $22-27-27$ & 50.8 & $\begin{array}{l}\text { gray-brown silt w/few pebbles, stones, } \\
\text { dry, hard, brittle, all grains dolo- } \\
\text { mite, gray mottling }\end{array}$ \\
\hline
\end{tabular}

\begin{tabular}{llllll}
\hline 4 & 805136 & $23.32-23.77$ & $24-30-25$ & 35.6 & $\begin{array}{l}\text { gray-brown silt w/few pebbles, stones, } \\
\text { dry, hard, brittle }\end{array}$ \\
\hline 4805137 & $23.77-24.23$ & $34-42-68$ & 40.6 & $\begin{array}{l}\text { gray-brown silt to } 23.88 \mathrm{~m} \text {; contact } \\
\text { w/gray-brown silt, no pebbles, stones } \\
\text { (clean), dry, very hard }\end{array}$ \\
\hline
\end{tabular}

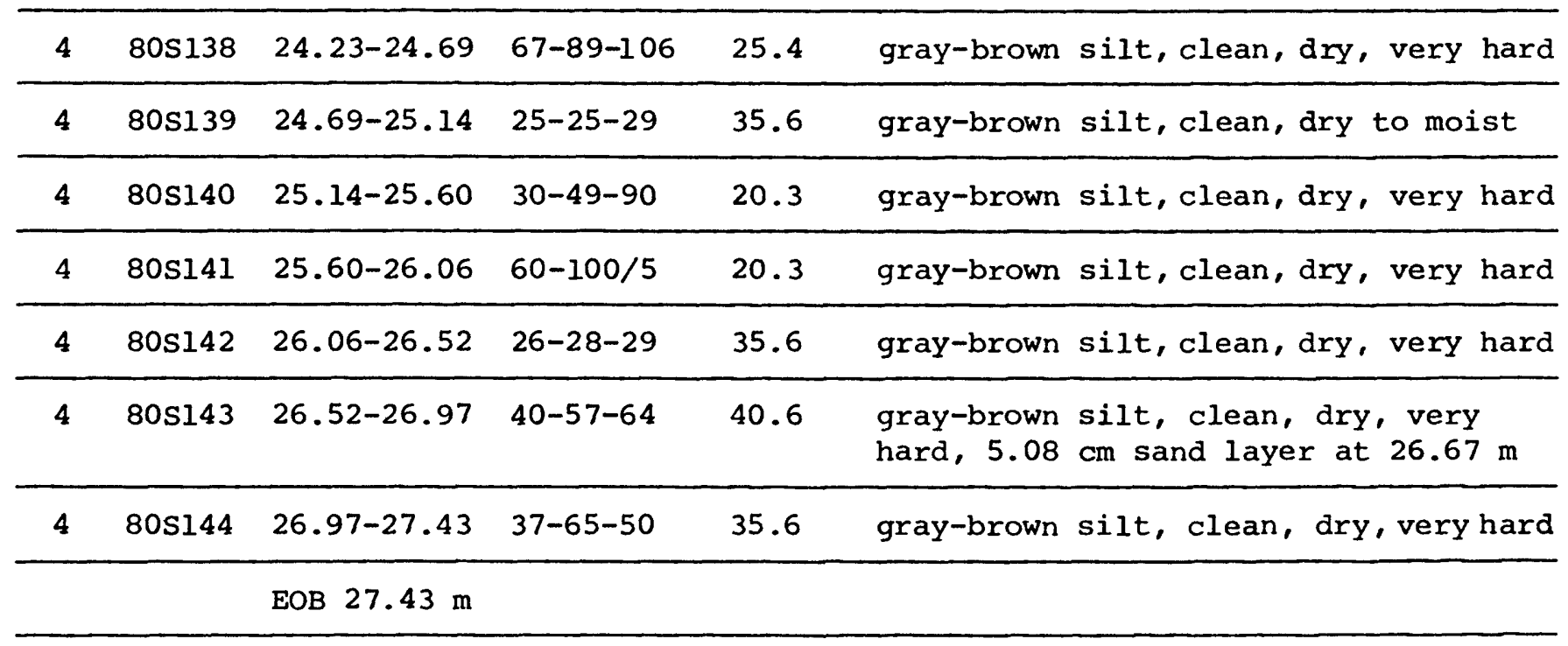


Table 2.--Test-well construction data and lithologic description of cores at Plot M--Continued

Test well 34

Completion date: June 12, 1980

Location: Next to test wells 4,35

Site and well construction data:

Altitude of land surface . . . . . . . . . $207.76 \mathrm{~m}$

Altitude of measuring point (top of casing). . . . . $207.80 \mathrm{~m}$

Depth of bottom of piezometer from land surface. . . $27.43 \mathrm{~m}$

Type of piezometer: $1.22 \mathrm{~m}$ slotted PVC pipe, $5.08 \mathrm{~cm}$ diameter

No samples collected during drilling. 
Table 2.--Test-well construction data and lithologic description of cores at Plot M--Continued

Test well 35

Completion date: June 23, 1980

Location: Next to test wells 4,34

Site and well construction data:

Altitude of land surface . . . . . . . . . . . $207.85 \mathrm{~m}$

Altitude of measuring point (top of casing). . . . $208.00 \mathrm{~m}$

Depth to bottom of piezometer from land surface. . . $33.38 \mathrm{~m}$

Type of piezometer: $1.22 \mathrm{~m}$ slotted PVC pipe, $5.08 \mathrm{~cm}$ diameter

\begin{tabular}{cccccc}
\hline $\begin{array}{c}\text { Unit } \\
\text { No. }\end{array}$ & $\begin{array}{c}\text { Sample } \\
\text { No. }\end{array}$ & $\begin{array}{c}\text { Depth } \\
\text { interval } \\
\text { (meters) }\end{array}$ & $\begin{array}{c}\text { Blow } \\
\text { counts }\end{array}$ & $\begin{array}{c}\text { Recovery } \\
(\mathrm{cm})\end{array}$ & Description \\
\hline 1 & 805145 & $0.15-0.60$ & $3-3-5$ & 15.2 & black topsoil, yellow silty clay \\
\hline 1 & 805146 & $0.60-1.07$ & $5-8-12$ & 25.4 & $\begin{array}{l}\text { yellow-brown clay w/pebbles, plant } \\
\text { fragments oxidized, moist, hard, wide } \\
\text { variety of rock and mineral grains }\end{array}$ \\
\hline
\end{tabular}

$\begin{array}{llllll}1 & 805147 & 1.07-1.52 & 14-22-21 & 30.5 & \begin{array}{l}\text { yellow-brown clay w/pebbles, stones, } \\ \text { oxidized, dry to moist, hard }\end{array}\end{array}$

\begin{tabular}{lllllll}
\hline 1 & 805148 & $1.52-1.98$ & $23-27-28$ & NR & \\
\hline 1 & $80 S 149$ & $1.98-2.44$ & $10-14-15$ & NR & \\
\hline 1 & 805150 & $2.44-2.90$ & $15-16-21$ & 10.2 & $\begin{array}{l}\text { yellow-brown silty clay w/pebbles, } \\
\text { stones, oxidized, moist, soft }\end{array}$ \\
\hline 1 & 805151 & $2.90-3.35$ & $5-7-10$ & 15.2 & $\begin{array}{l}\text { yellow-brown silty clay w/pebbles, } \\
\text { stones, oxidized, moist, wide variety } \\
\text { of rock and mineral grains including } \\
\text { shale, granite, dolomite, pyrite, } \\
\text { quartz, mica, blue-green (Cu) and } \\
\text { orange (Fe) mottling }\end{array}$ \\
\hline 1 & 805152 & $3.35-3.81$ & $10-12-15$ & 25.4 & $\begin{array}{l}\text { yellow-brown silty clay w/pebbles, } \\
\text { stones, oxidized, moist, abundant } \\
\text { black shale grains }\end{array}$ \\
\hline
\end{tabular}


Table 2.--Test-well construction data and lithologic description of cores at Plot M--Continued

Test well 35--Continued

\begin{tabular}{cccccc}
\hline $\begin{array}{c}\text { Unit } \\
\text { No. }\end{array}$ & $\begin{array}{c}\text { Sample } \\
\text { No. }\end{array}$ & $\begin{array}{c}\text { Depth } \\
\text { interval } \\
\text { (meters) }\end{array}$ & $\begin{array}{c}\text { Blow } \\
\text { counts }\end{array}$ & $\begin{array}{c}\text { Recovery } \\
(\mathrm{cm})\end{array}$ & \multicolumn{1}{c}{ Description } \\
\hline 1 & 805153 & $3.81-4.27$ & $7-11-13$ & 30.5 & $\begin{array}{l}\text { brown silty clay w/pebbles, stones; } \\
\text { grading downwards to gray-brown silty } \\
\text { clay w/pebbles, stones, abundant } \\
\text { pyrite }\end{array}$ \\
\hline 2 & 805154 & $4.27-4.72$ & $9-10-14$ & 33.0 & $\begin{array}{l}\text { gray-brown silty clay w/pebbles, few } \\
\text { stones, dry to moist, soft, mostly } \\
\text { dolomite and black shale grains }\end{array}$ \\
\hline 2 & 805155 & $4.72-5.18$ & $20-30-28$ & 25.4 & $\begin{array}{l}\text { gray-brown silty clay to 4.88 m; } \\
\text { contact w/water-bearing sand, } 5.1 \mathrm{~cm} \\
\text { thick }\end{array}$ \\
\hline 2 & 805156 & $5.18-5.64$ & $9-11-16$ & 35.6 & $\begin{array}{l}\text { 10.16 cm water-bearing sand to } 5.27 \mathrm{~m} ; \\
\text { contact w/gray-brown clayey silt w/ } \\
\text { pebbles, few stones }\end{array}$ \\
\hline
\end{tabular}

\begin{tabular}{ccccccl}
\hline 2 & 805157 & $5.64-6.10$ & $9-14-20$ & 15.2 & $\begin{array}{l}\text { gray-brown clayey silt w/pebbles, few } \\
\text { stones and rocks, moist, soft, mostly } \\
\text { dolomite, shale, pyrite grains }\end{array}$ \\
\hline 2 & 805158 & $6.10-6.54$ & $17-20-20$ & 40.6 & $\begin{array}{l}\text { gray-brown clayey silt w/pebbles, few } \\
\text { stones, moist, hard }\end{array}$ \\
\hline 2 & 805159 & $6.54-7.01$ & $12-19-24$ & 35.6 & $\begin{array}{l}\text { gray-brown clayey silt w/pebbles, few } \\
\text { stones, moist, hard }\end{array}$ \\
\hline 2 & 805160 & $7.01-7.47$ & $16-24-24$ & 25.4 & $\begin{array}{l}\text { gray-brown clayey silt w/pebbles, few } \\
\text { stones, moist, hard }\end{array}$ \\
\hline 2 & 805161 & $7.47-7.93$ & $19-26-14$ & 30.5 & $\begin{array}{l}\text { gray-brown clayey silt w/pebbles to } \\
\text { g.49 m; contact w/water-bearing sand, } \\
\text { lo.2 cm thick to } 7.59 \mathrm{~m} \text { contact w/ } \\
\text { gray-brown clayey silt w/pebbles, few } \\
\text { stones }\end{array}$ \\
\hline 2 & 805162 & $7.93-8.38$ & $14-20-27$ & 35.6 & $\begin{array}{l}\text { gray-brown clayey silt w/few pebbles, } \\
\text { stones }\end{array}$ \\
\hline
\end{tabular}


Table 2.--Test-well construction data and lithologic description of cores at Plot M--Continued

Test well 35 --Continued

\begin{tabular}{cccccl}
\hline $\begin{array}{l}\text { Unit } \\
\text { No. }\end{array}$ & $\begin{array}{c}\text { Sample } \\
\text { No. }\end{array}$ & $\begin{array}{c}\text { Depth } \\
\text { interval } \\
\text { (meters) }\end{array}$ & $\begin{array}{c}\text { Blow } \\
\text { counts }\end{array}$ & $\begin{array}{c}\text { Recovery } \\
(\mathrm{cm})\end{array}$ & \multicolumn{1}{c}{ Description } \\
\hline 2 & $80 \mathrm{~s} 163$ & $8.38-8.84$ & $12-22-48$ & 10.2 & $\begin{array}{l}\text { gray-brown clayey silt w/few pebbles, } \\
\text { stones }\end{array}$ \\
\hline 2 & 805164 & $8.84-9.30$ & $11-18-25$ & 20.3 & $\begin{array}{l}\text { gray-brown clayey silt w/few pebbles } \\
\text { to } 8.99 \mathrm{~m} \text {; contact w/brown clayey } \\
\text { silt w/pebbles, stones, moist, soft, } \\
\text { gritty, mostly dolomite, black shale, } \\
\text { green shale grains }\end{array}$ \\
\hline
\end{tabular}

\begin{tabular}{|c|c|c|c|c|c|}
\hline 2 & $80 S 165$ & $9.30-9.75$ & $20-25-24$ & 15.2 & $\begin{array}{l}\text { brown clayey silt } w / \text { pebbles, stones; } \\
\text { grading downwards to brown clayey } \\
\text { silt w/few pebbles, stones }\end{array}$ \\
\hline 2 & 805166 & $9.75-10.21$ & $14-17-21$ & 25.4 & $\begin{array}{l}\text { brown clayey silt } w / \text { few pebbles, } \\
\text { stones, moist, soft }\end{array}$ \\
\hline 2 & 805167 & $10.21-10.67$ & $8-10-12$ & 30.5 & $\begin{array}{l}\text { brown clayey silt w/few pebbles, } \\
\text { moist, soft to } 10.52 \mathrm{~m} \text {; contact w/ } \\
\text { pink clayey silt } 5.1 \mathrm{~cm} \text { thick; con- } \\
\text { tact w/brown clayey silt w/abundant } \\
\text { pebbles, stones, mostly black shale } \\
\text { and dolomite }\end{array}$ \\
\hline 2 & $80 S 168$ & $10.67-11.13$ & $8-13-12$ & 25.4 & $\begin{array}{l}\text { brown clayey silt w/pebbles, stones, } \\
\text { moist, very soft }\end{array}$ \\
\hline 2 & $80 \$ 169$ & $11.13-11.58$ & $7-7-10$ & 35.6 & $\begin{array}{l}\text { brown clayey silt w/pebbles, stones, } \\
\text { red-brown mottling to } 11.28 \mathrm{~m} \text {; con- } \\
\text { tact w/red-brown sandy silt, } 5.1 \mathrm{~cm} \\
\text { thick, to } 11.33 \mathrm{~m} \text {; contact w/brown } \\
\text { clayey silt, } 5.1 \mathrm{~cm} \text { thick, to } 11.38 \\
\text { m; contact w/red-brown sandy silt }\end{array}$ \\
\hline 2 & $80 S 170$ & $11.58-12.04$ & $9-17-33$ & 40.6 & $\begin{array}{l}\text { brown clayey silt w/pebbles, moist, } \\
\text { soft, mostly dolomite and shale } \\
\text { grains, to } 11.89 \mathrm{~m} \text {; contact w/wet } \\
\text { gravel } 5.08 \mathrm{~cm} \text { thick, and brown silt } \\
\text { and gravel }\end{array}$ \\
\hline
\end{tabular}


Table 2.--Test-well construction data and lithologic description of cores at Plot M--Continued

Test well 35--Continued

\begin{tabular}{cccccl}
\hline $\begin{array}{c}\text { Unit } \\
\text { No. }\end{array}$ & $\begin{array}{c}\text { Sample } \\
\text { No. }\end{array}$ & $\begin{array}{c}\text { Depth } \\
\text { interval } \\
\text { (meters) }\end{array}$ & $\begin{array}{c}\text { Blow } \\
\text { counts }\end{array}$ & $\begin{array}{c}\text { Recovery } \\
(\mathrm{cm})\end{array}$ & \multicolumn{1}{c}{ Description } \\
\hline 2 & 805171 & $12.04-12.50$ & $19-13-11$ & 25.4 & $\begin{array}{l}\text { gray-brown clayey silt to 12.19 m; } \\
\text { contact w/sandy gravel 5.1 cm thick } \\
\text { to 12.24 m contact w/brown clayey } \\
\text { silt w/pebbles, soft, mostly dolomite } \\
\text { grains }\end{array}$ \\
\hline 2 & 805172 & $12.50-12.95$ & $9-21-38$ & 7.6 & brown clayey silt, soft \\
\hline 2 & 805173 & $12.95-13.41$ & $18-21-26$ & $\mathrm{NR}$ & \\
\hline 3 & 805174 & $13.41-13.87$ & $10-16-19$ & 15.2 & $\begin{array}{l}\text { gray-brown silt, few pebbles, moist, } \\
\text { soft }\end{array}$ \\
\hline
\end{tabular}

\begin{tabular}{llllll}
\hline 3 & $80 S 175$ & $13.87-14.33$ & $14-19-22$ & NR & \\
\hline 3 & 805176 & $14.33-14.78$ & $8-18-20$ & 35.6 & $\begin{array}{l}\text { gray-brown silt w/few pebbles, moist, } \\
\text { soft, mostly dolomite and black shale } \\
\text { grains }\end{array}$ \\
\hline
\end{tabular}

$3805177 \quad 14.78-15.24 \quad 30-35-35 \quad 61.0$ brown clayey silt w/few pebbles, dry to moist, hard, brittle, nearly all grains are dolomites

\begin{tabular}{lllllll}
\hline 3 & $80 S 178$ & $15.24-15.70$ & NR & \\
\hline 3 & $80 S 179$ & $15.70-16.15$ & $26-28-31$ & 61.0 & $\begin{array}{l}\text { brown clayey silt w/few pebbles, dry } \\
\text { to moist, hard }\end{array}$ \\
\hline 3 & 805180 & $16.15-16.61$ & & NR & \\
\hline 3 & 805181 & $16.61-17.07$ & $6-13-18$ & 30.5 & $\begin{array}{l}\text { brown clayey silt w/few pebbles } \\
\text { (clean), moist, soft, all grains } \\
\text { dolomite }\end{array}$ \\
\hline 3 & 805182 & $17.07-17.52$ & $21-22-31$ & 61.0 & $\begin{array}{l}\text { brown clayey silt w/pebbles; grading } \\
\text { to gray silt, clean, moist }\end{array}$ \\
\hline 3 & 805183 & $17.52-17.98$ & $21-23-24$ & 40.6 & $\begin{array}{l}\text { gray silt, clean, moist; grading down- } \\
\text { wards to gray-brown silt and gravel }\end{array}$ \\
\hline
\end{tabular}


Table 2.--Test-well construction data and lithologic description of cores at Plot M--Continued

Test well $35--$ Continued

\begin{tabular}{|c|c|c|c|c|c|}
\hline $\begin{array}{l}\text { Unit } \\
\text { No. }\end{array}$ & $\begin{array}{l}\text { Sample } \\
\text { No. }\end{array}$ & $\begin{array}{c}\text { Depth } \\
\text { interval } \\
\text { (meters) }\end{array}$ & $\begin{array}{l}\text { Blow } \\
\text { counts }\end{array}$ & $\begin{array}{l}\text { Recovery } \\
\quad(\mathrm{cm})\end{array}$ & Description. \\
\hline 3 & $80 S 184$ & $17.98-18.44$ & $18-21-26$ & 45.7 & $\begin{array}{l}\text { gray-brown silt } w / \text { pebbles, stones, to } \\
18.07 \mathrm{~m} \text {; contact } \mathrm{w} / \mathrm{gray} \text {-brown silt, } \\
\text { clean, moist, soft }\end{array}$ \\
\hline 4 & $80 S 185$ & $18.44-18.90$ & $23-30-37$ & 35.6 & gray-brown silt, clean, moist, soft \\
\hline 4 & $80 S 186$ & $18.90-19.36$ & $13-16-18$ & 38.1 & gray-brown silt, clean, moist, soft \\
\hline 4 & $80 S 187$ & $19.36-19.81$ & $21-27-60$ & 35.6 & $\begin{array}{l}\text { gray-brown silt, very clean; grading } \\
\text { downwards to gray-brown silt, clean, } \\
\text { dry to moist, hard, brittle }\end{array}$ \\
\hline 4 & 805188 & $19.81-20.27$ & $36-32-44$ & NR & \\
\hline 4 & $80 \$ 189$ & $20.27-20.73$ & $92-51-52$ & 15.2 & $\begin{array}{l}\text { rock in sampler, gray-brown silt, } \\
\text { clean, hard to } 20.36 \mathrm{~m} \text {; contact w/ } \\
\text { brown silt, gritty, moist, soft }\end{array}$ \\
\hline 4 & $80 S 190$ & $20.73-21.18$ & $12-13-15$ & 35.6 & brown silt, gritty, moist, soft \\
\hline 4 & $80 S 191$ & $21.18-21.64$ & $22-17-16$ & 7.6 & brown silt, gritty, moist, soft \\
\hline 4 & $80 S 192$ & $21.64-22.10$ & $24-23-22$ & 35.6 & brown silt, gritty, moist, soft \\
\hline 4 & 805193 & $22.10-22.56$ & $23-25-32$ & NR & \\
\hline 4 & $80 S 194$ & $22.56-23.01$ & $12-13-20$ & 38.1 & brown silt, gritty, moist, soft \\
\hline 4 & $80 S 195$ & $23.01-23.47$ & $27-29-30$ & 40.6 & $\begin{array}{l}\text { brown silt, gritty, moist, soft to } \\
23.10 \mathrm{~m} \text {; contact w/gray-brown silt } \\
\text { w/few pebbles, dry to moist, hard }\end{array}$ \\
\hline 5 & 805196 & $23.47-23.93$ & $14-28-41$ & 35.6 & $\begin{array}{l}\text { gray-brown silt, no pebbles (clean), } \\
\text { very dry, hard }\end{array}$ \\
\hline 5 & $80 S 197$ & $23.93-24.38$ & $36-40-41$ & 40.6 & $\begin{array}{l}\text { gray-brown silt, clean, very dry, } \\
\text { hard, fresh dolomite rock in sampler }\end{array}$ \\
\hline 5 & $80 \$ 198$ & $24.38-24.84$ & $36-42-100 / 5$ & 530.5 & $\begin{array}{l}\text { gray-brown silt w/few rocks, dry, } \\
\text { very hard }\end{array}$ \\
\hline
\end{tabular}


Table 2.--Test-well construction data and lithologic description of cores at Plot $\mathrm{M}$--Continued

Test well 35--Continued

\begin{tabular}{|c|c|c|c|c|c|}
\hline $\begin{array}{l}\text { Unit } \\
\text { No. }\end{array}$ & $\begin{array}{l}\text { Sample } \\
\text { No. }\end{array}$ & $\begin{array}{c}\text { Depth } \\
\text { interval } \\
\text { (meters) }\end{array}$ & $\begin{array}{l}\text { Blow } \\
\text { counts }\end{array}$ & $\begin{array}{l}\text { Recovery } \\
\quad(\mathrm{cm})\end{array}$ & Description \\
\hline 5 & 805199 & $24.84-25.30$ & & NR & \\
\hline 5 & 805200 & $25.30-25.76$ & $22-35-37$ & 35.6 & $\begin{array}{l}\text { gray-brown silt w/few rocks, dry, } \\
\text { hard }\end{array}$ \\
\hline 5 & 805201 & $25.76-26.21$ & $52-34-29$ & 20.3 & $\begin{array}{l}\text { rock in sampler, gray-brown silt w/ } \\
\text { few rocks, dry, hard }\end{array}$ \\
\hline 5 & 805202 & $26.21-26.70$ & $22-29-34$ & 35.6 & $\begin{array}{l}\text { gray-brown silt w/few rocks, dry, } \\
\text { hard, flaky }\end{array}$ \\
\hline 5 & 805203 & $26.70-27.13$ & $30-22-22$ & 45.7 & $\begin{array}{l}\text { gray-brown silt w/few rocks, dry, } \\
\text { hard, flaky }\end{array}$ \\
\hline 5 & 805204 & $27.13-27.58$ & $15-17-27$ & 50.8 & $\begin{array}{l}\text { gray-brown silt; grading downward to } \\
\text { gray-brown silt w/sand, moist to wet, } \\
\text { stiff, flaky }\end{array}$ \\
\hline 5 & 805205 & $27.58-28.04$ & $77-100 / 4$ & 61.0 & $\begin{array}{l}\text { gray-brown silt; grading downward to } \\
\text { gray-brown silt w/sand; grading down- } \\
\text { ward to light brown silty sand, dry, } \\
\text { very hard, flaky }\end{array}$ \\
\hline 5 & 805206 & $28.04-28.50$ & $59-89-100 / 5$ & 535.6 & $\begin{array}{l}\text { light brown silty sand, dry, very } \\
\text { hard, flaky }\end{array}$ \\
\hline 5 & 805207 & $28.50-28.96$ & $53-69-100 / 5$ & 15.2 & $\begin{array}{l}\text { light brown silty sand, dry, very } \\
\text { hard, no pebbles, brittle, flaky }\end{array}$ \\
\hline 5 & $80 S 208$ & $28.96-29.41$ & $51-35-36$ & 35.6 & $\begin{array}{l}\text { light brown silty sand to } 29.02 \mathrm{~m} \text {; } \\
\text { contact w/wet silty sand, } 12.7 \mathrm{~cm} \\
\text { thick; grading to sandy silt }\end{array}$ \\
\hline 5 & $80 \$ 209$ & $29.41-29.87$ & $98-100 / 5$ & 15.2 & $\begin{array}{l}\text { light brown silty sand } w / \text { several thin } \\
\text { sand partings, dry, very hard }\end{array}$ \\
\hline 5 & 805210 & $29.87-30.33$ & $21-30-30$ & 30.5 & $\begin{array}{l}\text { light brown silty sand to } 30.02 \mathrm{~m} \text {; } \\
\text { contact } w / \text { white dolomite gravel, sand, } \\
\text { wet, soft, loose }\end{array}$ \\
\hline
\end{tabular}


Table 2.--Test-well construction data and lithologic description of cores at Plot M--Continued

\section{Test well 35--Continued}

\begin{tabular}{cccccl}
\hline $\begin{array}{c}\text { Unit } \\
\text { No. }\end{array}$ & $\begin{array}{c}\text { Sample } \\
\text { No. }\end{array}$ & $\begin{array}{c}\text { Depth } \\
\text { interval } \\
\text { (meters) }\end{array}$ & $\begin{array}{c}\text { Blow } \\
\text { counts }\end{array}$ & $\begin{array}{c}\text { Recovery } \\
(\mathrm{cm})\end{array}$ & \multicolumn{1}{c}{ Description } \\
\hline 5 & 805211 & $30.33-30.78$ & $7-9-11$ & 20.3 & $\begin{array}{c}\text { white sand to 30.4l m; contact w/gray } \\
\text { gravelly silt w/pebbles, stones, } \\
\text { moist to wet, soft }\end{array}$ \\
\hline 6 & 805212 & $30.78-31.24$ & $30-12-8$ & 15.2 & gray gravelly silt w/stones \\
\hline 6 & 805213 & $31.24-31.70$ & $10-25-18$ & NR & \\
\hline 6 & 805214 & $31.70-32.16$ & $11-20-30$ & 15.2 & gray gravelly silt w/stones \\
\hline 6 & 805215 & $32.16-32.61$ & $100 / 0$ & NR & rocks \\
\hline 6 & 805216 & $32.61-33.07$ & $100 / 6$ & NR & rocks \\
\hline 6 & 805217 & $33.07-33.52$ & $100 / 0$ & NR & rocks and bedrock (?) \\
\hline
\end{tabular}


Table 2.--Test-well construction data and lithologic description of cores at Plot M--Continued

Test well 36

Completion date: June 30,1980

Location: Next to test well 26

Site and well construction data:

Altitude of land surface . . . . . . . . . $210.77 \mathrm{~m}$

Altitude of measuring point (top of casing). . . . $210.92 \mathrm{~m}$

Depth to bottom of piezometer from land surface. . . $38.71 \mathrm{~m}$

Type of piezometer: $1.22 \mathrm{~m}$ slotted PVC pipe, $5.08 \mathrm{~cm}$ diameter

\begin{tabular}{cccccc}
\hline $\begin{array}{l}\text { Unit } \\
\text { No. }\end{array}$ & $\begin{array}{c}\text { Sample } \\
\text { No. }\end{array}$ & $\begin{array}{c}\text { Depth } \\
\text { interval } \\
\text { (meters) }\end{array}$ & $\begin{array}{c}\text { Blow } \\
\text { counts }\end{array}$ & $\begin{array}{c}\text { Recovery } \\
\text { (cm) }\end{array}$ & Description \\
\hline 1 & 805218 & $1.22-1.68$ & $9-9-9$ & 30.5 & $\begin{array}{l}\text { yellow-brown clay w/pebbles, plant } \\
\text { fragments, moist, soft, wide variety } \\
\text { of rock and mineral grains }\end{array}$ \\
\hline 1 & 805219 & $2.74-3.20$ & $15-16-24$ & 35.6 & $\begin{array}{l}\text { brown clay w/pebbles, dry to moist, } \\
\text { hard, oxidized, numerous black shale } \\
\text { grains, several vertical fractures }\end{array}$ \\
\hline
\end{tabular}

\begin{tabular}{|c|c|c|c|c|c|}
\hline 1 & $80 \$ 220$ & $4.27-4.72$ & $8-9-12$ & 50.8 & $\begin{array}{l}\text { brown silty clay w/pebbles, mostly } \\
\text { dolomite, black and green shale grains }\end{array}$ \\
\hline 1 & 805221 & $5.79-6.25$ & $12-34-34$ & 15.2 & $\begin{array}{l}\text { brown silty clay w/pebbles; grading } \\
\text { downward to gray-brown clayey silt } \\
\text { w/gravel, moist to wet }\end{array}$ \\
\hline 2 & $80 S 222$ & $7.32-7.77$ & $7-9-13$ & 40.6 & $\begin{array}{l}\text { gray-brown clayey silt } w / \text { pebbles, } \\
\text { stones, dry to moist, soft, mostly } \\
\text { dolomite and black shale grains }\end{array}$ \\
\hline 2 & 805223 & $8.84-9.29$ & $4-7-11$ & 40.6 & $\begin{array}{l}\text { gray-brown clayey silt w/pebbles, } \\
\text { stones, moist, soft }\end{array}$ \\
\hline 2 & 805224 & $10.36-10.82$ & $15-22-29$ & NR & \\
\hline 2 & 805225 & $11.89-12.34$ & $4-8-10$ & 35.5 & $\begin{array}{l}\text { gray-brown clayey silt w/numerous } \\
\text { pebbles, stones, moist, very soft, } \\
\text { mostly dolomite, black shale, pyrite }\end{array}$ \\
\hline
\end{tabular}


Table 2.--Test-well construction data and lithologic description of cores at Plot M--Continued

Test well 36--Continued

\begin{tabular}{cccccc}
\hline $\begin{array}{c}\text { Unit } \\
\text { No. }\end{array}$ & $\begin{array}{c}\text { Sample } \\
\text { No. }\end{array}$ & $\begin{array}{c}\text { Depth } \\
\text { interval } \\
\text { (meters) }\end{array}$ & $\begin{array}{c}\text { Blow } \\
\text { counts }\end{array}$ & $\begin{array}{c}\text { Recovery } \\
(\mathrm{cm})\end{array}$ & Description \\
\hline 2 & 805226 & $13.41-13.87$ & $7-9-11$ & 61.0 & $\begin{array}{l}\text { gray-brown clayey silt to 13.85 m; } \\
\text { contact w/pink clayey silt w/few peb- } \\
\text { bles, moist, soft, mostly dolomite } \\
\text { and black shale grains }\end{array}$ \\
\hline 2 & 805227 & $14.93-15.39$ & $8-18-25$ & 35.6 & $\begin{array}{l}\text { brown clayey silt w/numerous pebbles, } \\
\text { stones; grading downard to gray- } \\
\text { brown clayey silt w/few pebbles, } \\
\text { stones, dry to moist, soft }\end{array}$ \\
\hline
\end{tabular}

3805228 16.45-16.91 8-10-12 35.6 gray-brown clayey silt to $16.52 \mathrm{~m}$; contact $w /$ brown clayey silt $w /$ few pebbles, wet, soft, nearly all grains are dolomite

\begin{tabular}{|c|c|c|c|c|c|}
\hline 3 & 805229 & $17.98-18.44$ & $8-13-18$ & 35.6 & $\begin{array}{l}\text { gray-brown silt } w / \text { few pebbles, moist, } \\
\text { soft }\end{array}$ \\
\hline 3 & 805230 & $18.44-18.90$ & $17-18-25$ & 30.5 & $\begin{array}{l}\text { gray-brown silt } w / \text { few pebbles, dry to } \\
\text { moist }\end{array}$ \\
\hline 3 & 805231 & $18.90-19.35$ & $8-13-16$ & 50.8 & $\begin{array}{l}\text { gray-brown silt, no pebbles (clean), } \\
\text { dry to moist }\end{array}$ \\
\hline 3 & 805232 & $19.35-19.81$ & $6-14-15$ & 50.8 & gray-brown silt, clean, dry to moist \\
\hline 3 & 805233 & $19.81-20.27$ & $15-20-25$ & 40.6 & $\begin{array}{l}\text { gray-brown silt, clean, dry, hard, } \\
\text { brittle }\end{array}$ \\
\hline 3 & 805234 & $20.27-20.72$ & $10-22-11$ & 61.0 & $\begin{array}{l}\text { gray-brown silt, clean, dry, hard, } \\
\text { gravelly sand at } 20.50 \mathrm{~m}\end{array}$ \\
\hline 3 & 805235 & $20.72-21.18$ & $2-14-17$ & 25.4 & $\begin{array}{l}\text { gray-brown silt, clean, dry, hard, } \\
\text { wet sand and gravel at bottom of } \\
\text { sampler }\end{array}$ \\
\hline 3 & $80 S 236$ & $21.18-21.64$ & $14-19-13$ & 38.1 & $\begin{array}{l}\text { gray-brown silt to } 21.27 \mathrm{~m} \text {; contact } \\
\text { w/brown silt w/pebbles, moist to wet, } \\
\text { soft, gritty, all grains are dolomite }\end{array}$ \\
\hline
\end{tabular}


Table 2.--Test-well construction data and lithologic description of cores at Plot M--Continued

Test well 36 --Continued

\begin{tabular}{|c|c|c|c|c|c|}
\hline $\begin{array}{l}\text { Unit } \\
\text { No. }\end{array}$ & $\begin{array}{l}\text { Sample } \\
\text { No. }\end{array}$ & $\begin{array}{c}\text { Depth } \\
\text { interval } \\
\text { (meters) } \\
\end{array}$ & $\begin{array}{l}\text { Blow } \\
\text { count }\end{array}$ & $\begin{array}{l}\text { Recovery } \\
\quad(\mathrm{cm})\end{array}$ & Description \\
\hline 3 & $80 S 237$ & $21.64-22.10$ & $14-22-24$ & 15.2 & brown silt $w /$ few pebbles \\
\hline 3 & 805238 & $22.10-22.56$ & $20-16-21$ & 38.1 & brown silt $w /$ few pebbles \\
\hline 3 & 805239 & $22.56-23.01$ & $13-15-22$ & 50.8 & $\begin{array}{l}\text { brown silt to } 22.67 \mathrm{~m} \text {; contact w/ } \\
\text { gray-brown silt w/pebbles, stones, } \\
\text { dry to moist, brittle, stiff, all } \\
\text { grains are dolomite }\end{array}$ \\
\hline 3 & $80 S 240$ & $23.01-23.47$ & $23-26-30$ & 25.4 & $\begin{array}{l}\text { gray-brown silt w/pebbles, stones, } \\
\text { dry to moist }\end{array}$ \\
\hline 4 & $80 S 241$ & $23.47-23.92$ & $15-22-27$ & 30.5 & $\begin{array}{l}\text { gray-brown silt w/pebbles, stones, } \\
\text { rocks, dry }\end{array}$ \\
\hline 4 & 805242 & $23.92-24.38$ & & NR & \\
\hline 4 & 805243 & $24.38-24.84$ & $13-23-32$ & 20.3 & $\begin{array}{l}\text { gray-brown silt } \mathrm{w} / \mathrm{pebbles,} \mathrm{stones,} \\
\text { rocks, dry to } 24.41 \mathrm{~m} \text {; contact } \mathrm{w} / \\
\text { brown silt w/pebbles, stones, rocks, } \\
\text { dry to moist, all grains are dolomite }\end{array}$ \\
\hline 4 & $80 S 244$ & $24.84-25.30$ & & NR & \\
\hline 4 & $80 \$ 245$ & $25.30-25.76$ & $22-34-31$ & 30.5 & $\begin{array}{l}\text { gray-brown clayey silt w/pebbles, } \\
\text { stones, rocks, dry to moist, brittle, } \\
\text { stiff, hard, all grains are dolomite }\end{array}$ \\
\hline 4 & $80 S 246$ & $25.76-26.21$ & $25-28-29$ & 35.6 & $\begin{array}{l}\text { gray-brown clayey silt w/pebbles, } \\
\text { stones }\end{array}$ \\
\hline 4 & 805247 & $26.21-26.67$ & $18-26-39$ & 25.4 & $\begin{array}{l}\text { gray-brown clayey silt w/pebbles, } \\
\text { stones, dry, hard, gritty }\end{array}$ \\
\hline 4 & 805248 & $26.67-27.13$ & $42-33-48$ & 25.4 & $\begin{array}{l}\text { gray-brown silt w/few pebbles, stones } \\
\text { dry to moist, hard, brittle, stiff }\end{array}$ \\
\hline 4 & $80 \$ 249$ & $27.13-27.58$ & $42-38-33$ & 45.7 & $\begin{array}{l}\text { gray-brown silt w/few pebbles, stones, } \\
\text { dry, hard }\end{array}$ \\
\hline
\end{tabular}


Table 2.--Test-well construction data and lithologic description of cores at Plot M--Continued

Test well 36--Continued

\begin{tabular}{|c|c|c|c|c|c|}
\hline $\begin{array}{l}\text { Unit } \\
\text { No. }\end{array}$ & $\begin{array}{l}\text { Sample } \\
\text { No. }\end{array}$ & $\begin{array}{c}\text { Depth } \\
\text { interval } \\
\text { (meters) }\end{array}$ & $\begin{array}{l}\text { Blow } \\
\text { counts }\end{array}$ & $\begin{array}{l}\text { Recovery } \\
\quad(\mathrm{cm})\end{array}$ & Description \\
\hline 4 & $80 S 250$ & $27.58-28.04$ & $23-24-38$ & 15.2 & $\begin{array}{l}\text { gray-brown silt, no pebbles, stones } \\
\text { (clean), dry, hard }\end{array}$ \\
\hline 4 & 805251 & $28.04-28.50$ & $62-54-40$ & 35.6 & $\begin{array}{l}\text { gray-brown silt, clean, dry, very } \\
\text { hard }\end{array}$ \\
\hline 4 & $80 S 252$ & $28.50-28.96$ & $28-30-54$ & 25.4 & $\begin{array}{l}\text { gray-brown silt, clean, dry, hard, } \\
5.1 \mathrm{~cm} \text { sand layer at } 28.77 \mathrm{~m}\end{array}$ \\
\hline 4 & 805253 & $28.96-29.41$ & $20-25-37$ & 35.6 & $\begin{array}{l}\text { gray-brown sandy silt, dry, hard, } 2.5 \\
\mathrm{~cm} \text { sand layers at } 29.11 \mathrm{~m}, 29.32 \mathrm{~m}\end{array}$ \\
\hline 5 & $80 S 254$ & $29.41-29.87$ & $400-100 / 5$ & 25.4 & $\begin{array}{l}\text { gray-brown sandy silt, dry, very hard, } \\
\text { brittle, stiff }\end{array}$ \\
\hline 5 & $80 S 255$ & $29.87-30.33$ & $100 / 6$ & 4.0 & $\begin{array}{l}\text { light gray-brown sandy silt, dry to } \\
\text { moist, very hard, brittle, stiff }\end{array}$ \\
\hline 5 & $80 S 256$ & $30.33-30.78$ & $70-65-200 / 4$ & 7.5 & light gray sandy silt \\
\hline 5 & 805257 & $30.78-31.24$ & $100 / 3$ & NR & \\
\hline 5 & $80 \$ 258$ & $31.24-31.70$ & $37-67-90$ & 30.5 & $\begin{array}{l}\text { gray-brown silt, clean, dry to moist, } \\
\text { hard, brittle, stiff }\end{array}$ \\
\hline 5 & $80 S 259$ & $31.70-32.16$ & $22-36-52$ & 30.5 & gray-brown silt, clean, dry, hard \\
\hline 5 & 805260 & $32.16-32.61$ & $57-100 / 5$ & 15.2 & $\begin{array}{l}\text { gray-brown silt, clean, dry to moist, } \\
\text { hard }\end{array}$ \\
\hline 5 & 805261 & $32.61-33.07$ & $44-81-100 / 5$ & 525.4 & $\begin{array}{l}\text { light gray sandy silt, clean, moist } \\
\text { to wet, very hard }\end{array}$ \\
\hline 5 & $80 S 262$ & $33.07-33.52$ & $23-61-100 / 5$ & 25.4 & $\begin{array}{l}\text { light gray sandy silt, clean, moist } \\
\text { to wet, very hard }\end{array}$ \\
\hline 5 & 805263 & $33.52-33.99$ & $44-64-59$ & 35.6 & $\begin{array}{l}\text { light gray sandy silt, clean, dry, } \\
\text { hard }\end{array}$ \\
\hline
\end{tabular}


Table 2.--Test-well construction data and lithologic description of cores at Plot M--Continued

Test well 36--Continued

\begin{tabular}{cccccc}
\hline $\begin{array}{c}\text { Unit } \\
\text { No. }\end{array}$ & $\begin{array}{c}\text { Sample } \\
\text { No. }\end{array}$ & $\begin{array}{c}\text { Depth } \\
\text { interval } \\
\text { (meters) }\end{array}$ & $\begin{array}{c}\text { Blow } \\
\text { counts }\end{array}$ & $\begin{array}{c}\text { Recovery } \\
(\mathrm{cm})\end{array}$ & \multicolumn{1}{c}{ Description } \\
\hline 5 & 805264 & $33.99-34.44$ & $37-71-90$ & 20.3 & $\begin{array}{l}\text { light gray sandy silt to } 34.07 \mathrm{~m} ; \\
\text { contact w/gray-brown sandy silt w/ } \\
\text { pebbles, wet; grading downward to }\end{array}$ \\
& & & $\begin{array}{l}\text { gray-brown silt w/sand to } 34.19 \mathrm{~m} ; \\
\text { contact w/light gray gravelly silt } \\
\text { w/pebbles, stones, rocks, wet }\end{array}$ \\
\hline
\end{tabular}

\begin{tabular}{|c|c|c|c|c|c|}
\hline 5 & $80 S 265$ & $34.44-34.90$ & $22-23-10$ & 15.2 & $\begin{array}{l}\text { light gray gravelly silt } \mathrm{w} / \mathrm{sand} \text {, } \\
\text { gravel, all grains are fresh dolomite }\end{array}$ \\
\hline 6 & $80 S 266$ & $34.90-35.36$ & $7-10-27$ & 30.5 & $\begin{array}{l}\text { light gray gravelly silt } w / \text { pebbles, } \\
\text { stones, rocks, wet }\end{array}$ \\
\hline 6 & 805267 & $35.36-35.81$ & $51-41-42$ & 15.2 & $\begin{array}{l}\text { light gray gravelly silt w/pebbles, } \\
\text { stones, rocks }\end{array}$ \\
\hline 6 & $80 S 268$ & $35.81-36.27$ & $44-63-52$ & 35.6 & $\begin{array}{l}\text { light gray gravelly silt } \mathrm{w} / \text { pebbles, } \\
\text { stones, rocks, moist }\end{array}$ \\
\hline 6 & $80 S 269$ & $36.27-36.73$ & $40-35-36$ & 30.5 & $\begin{array}{l}\text { light gray gravelly silt w/pebbles, } \\
\text { stones, rocks }\end{array}$ \\
\hline 6 & 805270 & $36.73-37.18$ & $52-50-70$ & 20.3 & $\begin{array}{l}\text { light gray gravelly silt w/pebbles, } \\
\text { stones, rocks }\end{array}$ \\
\hline 6 & 805271 & $37.18-37.64$ & & NR & \\
\hline 7 & 805272 & $37.64-38.10$ & $15-37-52$ & 15.2 & $\begin{array}{l}\text { light gray gravelly silt to } 37.79 \mathrm{~m} \text {; } \\
\text { contact w/dark brown clayey silt w/ } \\
\text { pebbles, dry, hard }\end{array}$ \\
\hline 7 & 805273 & $38.10-38.55$ & & NR & \\
\hline 7 & 805274 & $38.55-38.86$ & $100 / 6$ & 15.2 & $\begin{array}{l}\text { dark brown clayey silt w/pebbles; } \\
\text { bedrock at } 38.86 \mathrm{~m}\end{array}$ \\
\hline
\end{tabular}

EOB $38.86 \mathrm{~m}$ 
Table 2.--Test-well construction data and lithologic description of cores at Plot M--Continued

\section{Test well 37}

Completion date: July 3, 1980

Location: Next to test wells 26,36

Site and well construction data:

Altitude of land surface . . . . . . . . . . $210.77 \mathrm{~m}$

Altitude of measuring point (top of casing). . . . . $210.92 \mathrm{~m}$ Depth to bottom of piezometer from land surface. . . . $27.28 \mathrm{~m}$

Type of piezometer: $1.22 \mathrm{~m}$ slotted PVC pipe, $5.08 \mathrm{~cm}$ diameter

No samples collected during drilling. 
Table 3.--Site and well construction data of bedrock test wells in the forest preserve near Plot $M$

$\mathrm{DH} 1$

Completion date: September 17, 1976

Location: Site $A$, at the top of the ridge in the Palos Forest Preserve

Site and well construction data (values are in meters):

Altitude of land surface ........... 226.46

Altitude of measuring point (top of casing). . . 227.06

Depth to bedrock from land surface... . . . . 51.81

Depth to bottom of casing from land surface. . . 53.34

Depth to bottom of borehole from land surface. . . 65.53

Type of casing: black steel, $12.7 \mathrm{~cm}$ diameter

DH2

Completion date: September 20, 1976

Location: 160 meters south of Plot $M$ on the north side of the forest preserve road; near SB2l

Site and well construction data (values are in meters):

Altitude of land surface........... . 219.79

Altitude of measuring point (top of casing). . . 220.39

Depth to bedrock from land surface....... . 46.93

Depth to bottom of casing from land surface. . . 48.77

Depth to bottom of borehole from land surface. . . 61.26

Type of casing: black steel, $12.7 \mathrm{~cm}$ diameter

DH3

Completion date: September 23, 1976

Location: 30 meters north-northwest of the northeast corner of Plot $M$; near SB4

Site and well construction data (values are in meters):

Altitude of land surface . . . . . . . . 207.08

Altitude of measuring point (top of casing). . . 207.68

Depth to bedrock from land surface . . . . . . 37.18

Depth to bottom of casing from land surface... . 39.01

Depth to bottom of borehole from land surface. . . 52.73

Type of casing: black steel, $12.7 \mathrm{~cm}$ diameter 
Table 3.--Site and well construction data of bedrock test wells in the forest preserve near Plot M--Continued

DH4

Completion date: September 28, 1976

Location: Approximately 200 meters north of Plot M next to forest preserve road

Site and well construction data (values are in meters):

Altitude of land surface........... 205.64

Altitude of measuring point (top of casing). . . 206.24

Depth to bedrock from land surface . . . . . . 33.22

Depth to bottom of casing from land surface. . . 35.35

Depth to bottom of borehole from land surface. . . 85.34

Type of casing: black steel, $12.7 \mathrm{~cm}$ diameter 